msh-mss Mathématiques et sciences humaines

185 | Printemps 2009

Varia

\title{
L'échec électoral est une science exacte
}

Failure of an election is scientifically predictable!

\section{Michel Fansten}

\section{OpenEdition}

Journals

Édition électronique

URL : http://journals.openedition.org/msh/11010

DOI : $10.4000 / \mathrm{msh} .11010$

ISSN : 1950-6821

\section{Éditeur}

Centre d'analyse et de mathématique sociales de l'EHESS

\section{Édition imprimée}

Date de publication : 1 mai 2009

Pagination : 37-67

ISSN : 0987-6936

\section{Référence électronique}

Michel Fansten, «L'échec électoral est une science exacte », Mathématiques et sciences humaines [En ligne], 185 | Printemps 2009, mis en ligne le 15 mai 2009, consulté le 23 juillet 2020. URL : http:// journals.openedition.org/msh/11010 ; DOI : https://doi.org/10.4000/msh.11010 


\title{
L’ÉCHEC ÉLECTORAL EST UNE SCIENCE EXACTE
}

\author{
Michel FANSTEN $^{1}$
}

\begin{abstract}
RÉSUMÉ - La représentation des comportements électoraux dont il va être question part d'un double postulat: ayant à choisir entre plusieurs candidats, un électeur vote pour celui dont il se considère le plus proche; dans un électorat homogène, la proximité ainsi introduite se distribue selon une loi statistique simple.

Nous avons présenté dans ce premier article quelques unes des applications de la formalisation mathématique ainsi obtenue dans le cas où les électeurs ont à choisir entre deux options ou deux candidats, en les illustrant à partir des élections françaises récentes. Elles montrent notamment qu'un électorat se mobilise d'autant moins que les choix qui lui sont proposés sont peu lisibles, ou que le camp $d u$ candidat dont il serait le plus proche est divisé. Dans une situation où le rapport des forces en présence est à peu près équilibré, cette abstention plus forte dans un camp que dans l'autre déciderait ainsi du résultat du scrutin.
\end{abstract}

MOTS-CLÉS - Abstention, Élection, Homogénéité de l'électorat, Lisibilité, Pondération des enjeux, Proximité

SUMMARY - Failure of an election is scientifically predictable !

Election behaviour, as represented in this paper, starts from a double hypothesis: given the choice of several candidates, a person will vote for the one he considers to be the closest to his or her views; within a homogeneous electorate, the factor of proximity is thus introduced according to simple statistical laws.

The present article demonstrates some of the applications of mathematical formulae obtained in the case in which voters have a choice between two options or two candidates as illustrated by the recent elections in France. They basically show that voters are all the less motivated when the choices are not clearly defined, or when their favourite candidate's side is divided. In the situation where the forces of power are more or less equal, it is the greater abstention on one side that will be the determining factor for election results Proximity

KEYWORDS - Abstention, Balance of power, Clarity, Election, Homogeneous electorate,

\section{INTRODUCTION}

Peut-on prévoir le résultat d'une élection?

Cet article a une histoire. Le 3 avril 2007, quelques semaines avant le premier tour de l'élection présidentielle française, un séminaire avait été organisé à l'EHESS sur le thème «les modélisations du vote». À cette occasion, j'avais été invité à présenter un travail théorique sur les comportements électoraux, qui avait donné lieu quelques mois plus tôt à la publication d'un article dans la Revue de science politique ${ }^{2}$.

\footnotetext{
${ }^{1}$ Administrateur INSEE (e.r.), michel.fansten@free.fr

2 «L'Indice de Condorcet. Représentation et analyse des comportements électoraux », Revue française de science politique, $\mathrm{n}^{\circ} 4$, août 2006.
} 
Les débats précédant mon intervention avaient tourné autour du problème de l'incidence du mode de scrutin sur le résultat d'une élection - illustré dans le contexte électoral français par le constat suivant : si François Bayrou était opposé au second tour, soit à Ségolène Royal, soit à Nicolas Sarkozy, les sondages donnaient à penser qu'il l'emporterait dans les deux cas. Or les mêmes sondages le donnaient, au premier tour, dépassé par l'un et par l'autre. Situation classique considérée par Condorcet, il y a plus de deux siècles, comme un «paradoxe »: «le candidat préféré à tous les autres, peut ne pas être élu» ${ }^{3}$.

Intervenant en fin de colloque, je me devais de situer mon approche par rapport à ce qui avait été dit. J'indiquais donc que, d'après le modèle dont il allait être question, Nicolas Sarkozy l'emporterait nettement au second tour sur Ségolène Royal, et que c'était justement parce que François Bayrou n'avait strictement aucune chance d'être présent à l'issue du premier tour, qu'il avait une telle position dans les sondages. Je me proposai d'en faire la démonstration ${ }^{4}$.

Ce qui avait surpris, c'était moins la prévision elle-même - à ce moment de la campagne électorale, elle correspondait à l'issue considérée comme la plus probable que l'assurance avec laquelle je la formulais, en parlant d'une nette victoire de Sarkozy, prévisible depuis plusieurs mois selon un modèle probabiliste simple.

J'indiquais d'ailleurs, puisqu'il avait été question de paradoxe, que cette prévision avait elle-même quelque chose de paradoxal : d'après le modèle théorique, le résultat d'une élection opposant deux candidats dans un contexte de bipolarisation de la vie politique devrait, en principe, être largement imprévisible : 50/50. Sauf précisément dans un cas, celui où un des deux candidats «s'est pris les pieds dans le tapis », en se laissant enfermer dans un positionnement qui ne lui permet pas de «faire le plein des voix de son camp ». Dans ce cas, ce sont les abstentionnistes, plus nombreux d'un côté que de l'autre, qui déterminent de fait le résultat de l'élection. J'ajoutais que curieusement cette configuration, où c'est une fraction politisée (sinon la plus politisée) de l'électorat qui décide de l'issue d'un scrutin en n'y participant pas, semblait être devenue la règle dans nos démocraties. Je n'envisageais à l'époque, ni les élections italiennes, ni l'élection présidentielle américaine...

\section{- Une approche différente de l'analyse et de la prévision des résultats électoraux}

Qu'est-ce qui détermine les résultats d'une élection?

Par rapport à l'abondante littérature à laquelle cette question donne lieu, il est probable que l'on retiendra d'abord de la démarche présentée ici la formalisation mathématique sur laquelle elle s'appuie, assez inhabituelle dans l'analyse des données d'un scrutin. Pourtant sa principale originalité tient moins à l'approche utilisée qu'à la grille d'analyse sur laquelle elle débouche.

Les résultats d'une élection se résument généralement aux pourcentages de voix obtenues par les candidats et au taux d'abstention. Présentés sous la forme de tableaux, de cartes, de graphiques, ils font apparaître les performances comparées des partis en

\footnotetext{
${ }^{3}$ Le paradoxe de Condorcet a donné lieu à une abondante littérature. On pourra notamment de référer à l'ouvrage de Jean-Louis Boursin : les paradoxes du vote, éd. Odile Jacob, février 2004.

${ }^{4}$ La démonstration en question avait d'ailleurs été déjà largement exposée six mois auparavant, dans l'article précité. De manière il est vrai plus prudente : "les candidats, le centre, et l'opinion moyenne », pp. 665 à 668 .
} 
présence, d'une circonscription à l'autre ou d'une élection à l'autre. Associés aux caractéristiques socio-économiques des populations concernées ou aux informations recueillies dans les enquêtes d'opinion, ils permettent de décrire et d'analyser les comportements des différentes catégories d'électeurs.

L'approche exposée dans cet article s'inscrit dans une problématique différente. Elle n'est pas en rupture avec les études menées depuis une trentaine d'années sur le sujet et elle fournit des résultats cohérents avec ceux qui ont été produits par d'autres modes d'analyse. Elle propose simplement d'autres paramètres, comme le positionnement relatif des candidats, la lisibilité des enjeux du scrutin, ou l'homogénéité de l'électorat.

Réduite à l'essentiel, la modélisation utilisée repose sur la proposition suivante : Ayant à choisir entre deux candidats, un électeur votera pour celui dont il se considère significativement le plus proche.

D'un point de vue mathématique, on associe ainsi le vote à ce qu'on peut appeler « une distance ». Il faut non seulement que la distance de l'électeur au candidat A soit plus petite que la distance au candidat $\mathrm{B}$, mais qu'elle le soit dans un rapport qui, pour être déterminant, doit être inférieur à un certain seuil. Sinon l'électeur, estimant que l'écart n'est pas suffisant, s'abstiendra ou votera blanc. La probabilité pour qu'un électeur vote en faveur de A est de ce fait fonction de ce que nous appellerons dans la suite de cet article «sa proximité relative par rapport à $\mathrm{A}$ ».

La manière d'apprécier de telles distances et le seuil à partir duquel elles sont perçues comme significativement différentes varient naturellement d'un électeur à l'autre. Le modèle de référence consiste à supposer que lorsque la population étudiée est homogène, la proximité se distribue selon une loi statistique simple : sa fréquence diminue au fur et à mesure qu'on s'éloigne de sa valeur moyenne.

Avant de développer ces différents points, il peut être important d'indiquer, d'emblée, que la représentation obtenue s'avère conforme à la réalité et met en évidence des tendances souvent masquées par la diversité des situations particulières.

Le modèle théorique débouche, par exemple, sur une proposition qui peut sembler surprenante : l'abstention est la plus forte dans les circonscriptions où le duel est le plus serré.

L'approche utilisée conduit alors à un autre constat: l'abstention affecte de manière inégale les forces en présence. En d'autres termes, plus l'abstention est forte, plus le risque est grand que le résultat d'un scrutin soit déterminé par les abstentionnistes, ou plus exactement, par un courant d'abstentions relativement plus marqué dans un camp que dans l'autre.

Nous montrerons par exemple que l'ampleur du succès de la gauche aux élections régionales de 2004 a tenu essentiellement à l'abstention d'une partie de l'électorat traditionnel de la droite, face à un scrutin dont les enjeux lui paraissaient peu lisibles. De même il semblerait que l'un des facteurs de la victoire du non au référendum de 2005 sur le projet de Constitution européenne, ait été la forte participation d'électeurs qui s'étaient abstenus lors des élections européennes de 2004, et qui ont voté majoritairement non. 
La formalisation théorique conduit de même à un certain nombre de propositions, comme : Une élection se gagne rarement au centre. La stratégie qui consiste à faire le plein des voix de son camp, c'est à dire à définir un positionnement qui assure sa cohésion, est généralement plus efficace que celle qui vise à convaincre les indécis. Ainsi formulée, une telle proposition peut paraître faire abstraction des situations politiques particulières. En réalité elle rend compte, comme nous le verrons, du réseau d'interactions à travers lequel ces situations déterminent le choix des électeurs.

J'ai tout à fait conscience, en écrivant cela, que l'utilisation de modèles mathématiques dans l'analyse ou la prévision des résultats électoraux fait généralement naître chez le lecteur un mélange de curiosité, de scepticisme et de méfiance.

Les objections soulevées lors de présentations des travaux dont il va être question sont, de ce point de vue, tout à fait significatives. Ainsi l'affirmation selon laquelle dans une élection opposant deux candidats, le taux d'abstention est le plus élevé là où le duel est le plus serré, provoque généralement une réaction d'incrédulité. Même si la tendance statistique mise en évidence sur les valeurs moyennes est indéniable, elle ne suffit pas à lever le doute. Le fait justement qu'il s'agisse d'une tendance moyenne alimente la réticence à admettre qu'un phénomène de cette nature puisse avoir un caractère général et ne pas résulter uniquement de circonstances politiques ou locales spécifiques.

On retrouve la même ambivalence face à une autre affirmation : quand deux candidats de centre droit et de centre gauche sont opposés l'un à l'autre, celui qui a le plus de chance de l'emporter est celui qui, face à un candidat d'extrême droite, aurait obtenu le plus de voix. Ce qui surprend, là encore, c'est moins la proposition elle-même - qui n'a rien de paradoxal - que la démonstration logique qui en ferait une sorte de règle générale, valable quelles que soient les conditions politiques du moment, ou la personnalité des candidats.

Il n'est donc pas inutile, à ce stade de l'introduction, de souligner que la modélisation introduite vise surtout à mettre en évidence des caractéristiques communes à des situations très différentes. Elle ne prétend pas rendre compte de toute la complexité des comportements électoraux : la carte n'est pas le paysage. D'autre part, et quelle que soit la généralité des tendances annoncées, il n'y a là aucun déterminisme. La grille d'analyse proposée permet d'ailleurs de repérer les situations dans lesquelles la prévision est particulièrement incertaine, comme celle que nous évoquerons à propos des ruptures dans le processus de bipolarisation, lorsque ce qui oppose les deux coalitions ayant vocation à exercer le pouvoir, devient moins déterminant que ce qui distingue entre elles leurs différentes composantes.

Enfin, il est important d'indiquer qu'il ne s'agit pas ici d'un travail de politologue. Des exemples concrets étaient nécessaires pour les besoins de l'exposé. La lecture qui en est faite semble renvoyer à des analyses politiques, mais là n'est pas son objet. Ces exemples sont destinés à illustrer ce que signifient, très concrètement, les tendances et les lois générales produites par le modèle théorique.

Nous exposerons, dans ce premier article, les principales caractéristiques du modèle de référence, établi dans le cas le plus simple d'un électorat ayant à choisir entre deux options ou deux candidats. Nous en présenterons les principales

\footnotetext{
5 Un prochain article présentera les extensions de ce modèle à des élections opposant un nombre quelconque de candidats.
} 
applications en montrant notamment, à propos de quelques élections françaises récentes, ce qu'apporte la prise en compte de la notion de "lisibilité des enjeux» et de son corollaire : "la fraction de l'électorat qui a la lecture la plus simple des enjeux est celle qui se mobilise le plus ».

Pour faciliter la lecture, les commentaires mettant en perspective les éléments théoriques figurent dans des encarts, de même que quelques formules qu'il était difficile de ne pas introduire à ce moment là. Les développements mathématiques qui soustendent la formalisation mise en cuvre ont été regroupés en annexe, ainsi que le détail des exemples cités.

Les lecteurs pressés ou peu familiers avec les représentations mathématiques utilisées, pourront toutefois survoler le texte et aller directement, p. 92, aux analyses sur lesquelles elles débouchent concernant les options stratégiques des candidats dans un contexte donné. Ce sont celles qui justifient le titre de cet article...

\section{LE MODÈLE THÉORIQUE DE RÉFÉRENCE : ÉLECTORAT HOMOGÈNE AYANT À CHOISIR ENTRE DEUX CANDIDATS, DANS UNE ÉLECTION COMPORTANT UN ENJEU UNIQUE}

Le modèle de référence repose sur deux hypothèses, dont chacune est la traduction d'une certaine forme de cohérence, caractérisant ce que nous avons appelé «un électorat homogène » :

- Première hypothèse : lorsque l'électorat a le choix entre deux candidats $\mathrm{A}$ et $\mathrm{B}$ et détermine son choix en fonction d'un seul enjeu, c'est-à-dire selon un critère unique de préférence, l'opinion de chaque électeur peut être représentée par un point sur axe - orienté par exemple de gauche à droite : plus ce point est à droite, plus l'opinion correspondante est favorable à $\mathrm{A}$ et défavorable à $\mathrm{B}$. Et dualement. La position de ce point définit « la proximité relative » de l'électeur à $\mathrm{A}$ par rapport à $\mathrm{B}$.

Cette première hypothèse traduit ce qu'on peut appeler la «cohérence logique » de l'électorat : les opinions de deux électeurs X et Y sont toujours comparables entre elles ; si $\mathrm{X}$ est à gauche de $\mathrm{Y}$, et $\mathrm{Y}$ à gauche de $\mathrm{Z}$, alors $\mathrm{X}$ est à gauche de $\mathrm{Z}$. Les préférences des électeurs sont ainsi, totalement ordonnées ${ }^{6}$.

- Seconde hypothèse : la distribution des opinions dans l'électorat est unimodale : leur fréquence décroît au fur et à mesure qu'on s'éloigne d'une position censée représenter l'opinion moyenne. Cette seconde hypothèse traduit ce qu'on peut appeler la «cohérence statistique » de l'électorat. Nous avons supposé, dans la construction du modèle de référence, que la distribution unimodale des proximités relatives pouvait être assimilée à une distribution normale ${ }^{7}$.

Dans cette représentation, le choix d'un électeur est fonction de sa proximité relative aux candidats A et B, sur l'axe caractérisant l'enjeu du scrutin. La proportion de voix obtenue par un candidat dans une circonscription donnée, est alors une estimation

\footnotetext{
${ }^{6}$ Une telle cohérence ne s'impose pas nécessairement pour chaque électeur pris isolément. Il suffit qu'elle s'exprime de manière statistique au niveau des groupes d'électeurs étudiés.

${ }^{7}$ Cette hypothèse complémentaire n'est pas indispensable - on aurait pu aussi bien utiliser d'autres lois unimodales de type stable par rapport à l'addition (comme les lois parétiennes) - mais elle simplifie considérablement la construction d'un modèle théorique et elle conduit à des représentations qui s'avèrent conformes aux résultats observés.
} 
de la probabilité que les électeurs de cette circonscription soient significativement plus proches de lui que de son concurrent.

Le score $\mathrm{N}(\mathrm{A})$ de $\mathrm{A}$ est ainsi déterminé par la proximité relative moyenne des électeurs à $\mathrm{A}$ par rapport à $\mathrm{B}$, (que nous désignerons plus simplement dans la suite de cet article par « la proximité moyenne de $\mathrm{A} »), \mathrm{P}_{\mathrm{A} / \mathrm{B},}$, selon la relation :

$$
\mathrm{N}(\mathrm{A})=\Pi\left(\mathrm{P}_{\mathrm{A} / \mathrm{B}}\right) \text { i.e. } \mathrm{P}_{\mathrm{A} / \mathrm{B}}=\Pi^{-1}[\mathrm{~N}(\mathrm{~A})]
$$

où $\Pi\left(\mathrm{P}_{\mathrm{A} / \mathrm{B}}\right)$ représente la fonction cumulative d'une loi normale pour la valeur $\mathrm{P}_{\mathrm{A} / \mathrm{B}}$.

La notion de proximité moyenne telle qu'elle vient d'être définie, est au centre de la représentation utilisée dans cette étude.

\section{- Représentation graphique du modèle de référence}

La répartition des électeurs en fonction de leurs proximités relatives aux deux candidats, A et B, peut être représentée sur un axe figurant l'enjeu de la consultation :

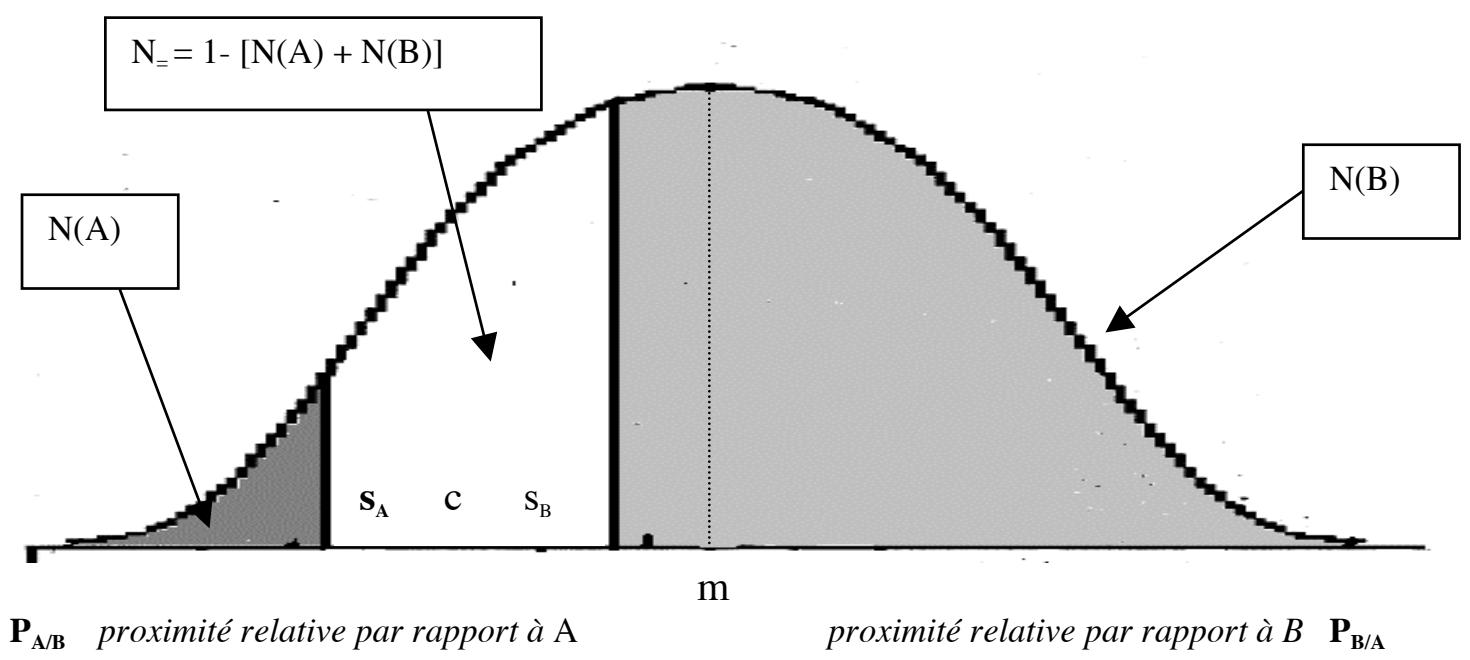

Le graphique ci-dessus représente la proportion d'électeurs en fonction de leur proximité à $\mathrm{A}$ ou à $\mathrm{B}$ (la proximité relative par rapport à $\mathrm{A}$ est, par définition, le négatif de la proximité relative par rapport à B). Les valeurs médianes sont figurées ici par un même point, $\mathrm{m}$, figurant la position de l'opinion moyenne. Les deux représentations sont symétriques et les graphiques correspondants, confondus.

Les deux points $\mathrm{s}_{\mathrm{A}}$ et $\mathrm{s}_{\mathrm{B}}$ figurent les seuils au-delà desquels un électeur vote pour $\mathrm{A}$, ou pour B. La distance $\mathrm{s}_{\mathrm{A}} \mathrm{s}_{\mathrm{B}}$ correspond à la zone d'indécision. Le point $\mathrm{c}$, au milieu de $\mathrm{S}_{\mathrm{A}} \mathrm{S}_{\mathrm{B}}$, figure la position du centre, c'est-à-dire celle des électeurs qui se situent à égale distance des deux candidats ${ }^{8}$.

$\mathrm{N}(\mathrm{A})$ représente la proportion d'électeurs ayant voté pour $\mathrm{A} . \mathrm{N}(\mathrm{B})$ représente la proportion d'électeurs ayant voté pour $\mathrm{B} . \mathrm{N}_{=}$représente la proportion d'électeurs qui,

\footnotetext{
${ }^{8}$ De ce point de vue, le centre correspond à la position moyenne des électeurs indécis.
} 
ne percevant pas de différence significative entre la position de $\mathrm{A}$ et celle de $\mathrm{B}$, ne se prononcent pas.

Le résultat d'un scrutin peut ainsi être analysé, très simplement, à partir des positions respectives de $\mathrm{s}_{\mathrm{A}}, \mathrm{s}_{\mathrm{B}}, \mathrm{c}, \mathrm{m}$.

Le candidat qui l'emporte est celui qui est le plus proche de l'opinion moyenne, $\mathrm{m}$. L'écart de scores $[\mathrm{N}(\mathrm{A})-\mathrm{N}(\mathrm{B})]$ entre les candidats est fonction de la distance entre $\mathrm{c}$ et $\mathrm{m}$ : quand les deux points sont confondus, les scores des candidats sont identiques.

La proportion d'abstentionnistes est d'autant plus élevée que le centre est proche de l'opinion moyenne. L'abstention augmente quand c se rapproche de $\mathrm{m}$, c'est-à-dire quand l'un des candidats se rapproche de son adversaire ou quand il se rapproche de l'opinion moyenne. Enfin, si on partage l'électorat en deux camps de part et d'autre de c (ceux qui sont plus proches de A et ceux qui sont plus proches de B), on observe que l'abstention ne se distribue pas de la même manière dans les deux camps.

\section{- Détermination des proximités moyennes}

Exemple : Deuxième tour de l'élection présidentielle de $2002^{9}$. Département de l'Ain

1. Résultats du scrutin

\begin{tabular}{|c|c|c|c|c|}
\hline Inscrits & Votants & $\begin{array}{c}\text { Suffrages } \\
\text { exprimés }\end{array}$ & Jacques Chirac & $\begin{array}{c}\text { Jean-Marie Le } \\
\text { Pen }\end{array}$ \\
\hline 338196 & 273803 & 258991 & 204031 & 54960 \\
\hline
\end{tabular}

\begin{tabular}{|c|c|}
\hline \multicolumn{2}{|c|}{ Pourcentages des suffrages exprimés } \\
\hline A : Chirac & B : Le Pen \\
\hline N(A) : $78,7 \%$ & N(A) $: 21,3 \%$ \\
\hline
\end{tabular}

2. Calcul des proximités moyennes

La proximité moyenne d'un candidat pour une circonscription donnée est déterminée par le pourcentage de voix obtenues par ce candidat, par rapport au nombre d'électeurs inscrits ${ }^{10}$.

\begin{tabular}{|c|c|c|}
\hline \multicolumn{3}{|c|}{ Pourcentages des inscrits } \\
\hline $\mathrm{N}($ Chirac $)$ & $\mathrm{N}=$ & $\mathrm{N}($ Le Pen $)$ \\
\hline $60,3 \%$ & $23,4 \%$ & $16,3 \%$ \\
\hline \multicolumn{3}{|c|}{ Proximités moyennes } \\
\hline \multicolumn{3}{|c|}{} \\
\hline Chirac & & Le Pen \\
\hline $\mathrm{P}_{\mathrm{A} / \mathrm{B}}: 0,262$ & & $\mathrm{P}_{\mathrm{B} / \mathrm{A}}:-0,984$ \\
\hline
\end{tabular}

\footnotetext{
${ }^{9}$ Le second tour de l'élection présidentielle de 2002, qui opposait des candidats très dissemblables, fournit des exemples particulièrement significatifs des applications de la représentation utilisée.

${ }^{10}$ À partir de la proportion $\mathrm{N}(\mathrm{A})$ d'électeurs ayant voté pour $\mathrm{A}$, « la proximité moyenne de $\mathrm{A}$ », $\mathrm{P}_{\mathrm{A} / \mathrm{B}}$, est donnée directement par Excel, comme fonction loi normale standard inverse de $\mathrm{N}(\mathrm{A}): \Pi\left(\mathrm{P}_{\mathrm{A} / \mathrm{B}}\right)=\mathrm{N}(\mathrm{A})$ La proximité moyenne de J. Chirac pour les électeurs de l'Ain est de 0,262: $\Pi(0,262)=60,3 \%$ Celle de J.-M. Le Pen est de - 0,984 : П(-0,984) = 16,3\%
} 
La somme des proximités moyennes des deux candidats donne la mesure de la zone d'indécision pour les électeurs de cette circonscription :

$$
\mathrm{P}_{\mathrm{A} / \mathrm{B}}+\mathrm{P}_{\mathrm{A} / \mathrm{B}}:-0,722
$$

Sa valeur est proche de sa valeur moyenne France entière : - 0,78

\section{I.1. « L'INDICE DE LISIBILITÉ »}

La formalisation qui vient d'être introduite débouche sur un certain nombre de propositions théoriques caractérisant un électorat homogène, c'est-à-dire tel que les proximités relatives des électeurs aux candidats en présence se distribuent selon une loi normale. La première d'entre elles découle de la définition des proximités moyennes :

Dans une élection opposant deux candidats, la somme de leurs proximités moyennes est constante d'une circonscription à l'autre et indépendante des scores des candidats $^{11}$.

$$
\mathrm{I}_{\mathrm{AB}}=-\left(\mathrm{P}_{\mathrm{A} / \mathrm{B}}+\mathrm{P}_{\mathrm{B} / \mathrm{A}}\right)
$$

- Un indicateur de la lisibilité des enjeux du scrutin

Par définition la somme des proximités moyennes caractérise la zone d'indécision, celle à l'intérieur de laquelle un électeur ne perçoit pas de différence significative entre les deux candidats : il s'en sent soit également proche, soit également éloigné. Une valeur élevée correspond ainsi à une implication faible de l'électorat et à une relative indifférence aux enjeux de la consultation.

Plus généralement, la valeur de l'indicateur obtenu augmente quand le positionnement des candidats est imprécis, quand les enjeux deviennent flous, ou quand l'électeur est tiraillé entre plusieurs logiques plus ou moins contradictoires - par exemple, quand l'alternative à laquelle il est confrontée est ambiguë : être pour A ne signifie pas nécessairement être contre B. Ces constats empiriques sont confirmés par l'analyse théorique. Nous montrerons en particulier que la multiplication des critères d'appréciation tend, en règle générale, à accroître la valeur de cet indicateur que, pour toutes ces raisons, nous appellerons dans la suite de cet article «indice de lisibilité ». Un indice de lisibilité élevé correspond à une faible lisibilité des enjeux du scrutin.

- Un indicateur de l'hétérogénéité de l'électorat

Si l'électorat est homogène au sens où nous l'avons défini, la somme des proximités moyennes est constante. Dans la réalité, elle fluctue plus ou moins d'une circonscription à l'autre. Un électorat n'est jamais parfaitement homogène : il l'est plus ou moins.

Nous montrerons que ces variations peuvent généralement être analysées comme résultant d'une partition au sein de l'électorat: des populations ayant des lectures différentes des enjeux ou ne votant pas en fonction des mêmes critères, et présentes dans des proportions variables dans les différentes circonscriptions.

\footnotetext{
${ }^{11} \mathrm{Cf}$. Annexe : le modèle de référence.

La somme $\mathrm{P}_{\mathrm{A} / \mathrm{B}}+\mathrm{P}_{\mathrm{B} / \mathrm{A}}$ est toujours négative. Par convention, l'indicateur $\mathrm{I}_{\mathrm{AB}}$ sera pris égal à la valeur absolue de la somme en question.
} 
Par convention, nous considérerons donc que le cœeficient de variabilité de l'indice de lisibilité, c'est-à-dire le rapport entre son écart-type et sa moyenne, caractérise l'hétérogénéité de l'électorat.

- L'exemple des seconds tours des élections présidentielles depuis 1981

- Une homogénéité plus grande :

Les résultats des deuxièmes tours des élections présidentielles département par département depuis $1981^{12}$, montrent que le cœfficient de variabilité de l'indice de lisibilité tend à diminuer d'une élection à l'autre. Pour reprendre les termes introduits précédemment : depuis 1981, l'électorat est devenu plus homogène même si, comme nous le verrons, une nouvelle forme d'hétérogénéité semble s'être manifestée en 2007.

\begin{tabular}{|c|c|c|c|c|}
\hline \multicolumn{5}{|c|}{ Variabilité de l'indice de lisibilité au second tour des élections présidentielles } \\
\hline 1981 & 1988 & 1995 & 2002 & 2007 \\
\hline 0,192 & 0,177 & 0,131 & 0,096 & 0,106 \\
\hline
\end{tabular}

À ce stade, deux explications peuvent être avancées. La première se situe au niveau des électeurs pris individuellement: les élections de ces dernières années s'inscrivent dans un contexte de bipolarisation de la vie politique qui s'accompagnerait d'une homogénéisation croissante des comportements. La seconde consiste à supposer que, même s'il subsiste chez les électeurs pris individuellement des différences importantes dans l'appréciation des enjeux des élections, les différences qui apparaissaient au niveau des départements se sont atténuées.

- Des lisibilités variables selon les candidats en présence :

La plupart des élections présidentielles affichent au second tour des lisibilités du même ordre, avec deux exceptions : 1969 et 2002.

\begin{tabular}{|c|c|c|c|c|c|c|c|}
\hline \multicolumn{6}{|c|}{ Valeur moyenne de l'indice de lisibilité aux seconds tours des présidentielles depuis 1965} \\
\hline 1965 & 1969 & 1974 & 1981 & 1988 & 1995 & 2002 & 2007 \\
\hline 0,40 & 0,81 & 0,31 & 0,34 & 0,38 & 0,48 & 0,78 & 0,47 \\
\hline
\end{tabular}

En 1969, le second tour opposait Georges Pompidou et Alain Poher qui, pour une partie des électeurs, présentaient un positionnement similaire (illustré par le mot du principal opposant de l'époque, le communiste Jacques Duclos : «bonnet blanc et blanc bonnet »). J'interprète la valeur élevée de l'indice de lisibilité selon la première raison évoquée précédemment: une implication faible de l'électorat et une relative indifférence aux enjeux de la consultation.

En 2002, en revanche, le second tour opposait deux candidats, Jacques Chirac et Jean-Marie Le Pen aux positionnements très contrastés. La valeur relativement forte de l'indice de lisibilité tiendrait ici à la seconde raison: En 1995, comme pour la plupart des élections précédentes, il s'agissait pour la majorité des électeurs d'une alternative simple : gauche droite. Voter contre l'un des candidats équivalait à voter pour l'autre.

\footnotetext{
${ }^{12} \mathrm{Cf}$. annexe : indices de lisibilité. On ne dispose pas de données statistiques facilement exploitables permettant de déterminer l'hétérogénéité de l'électorat pour les élections présidentielles avant 1981.
} 
En 2002 en revanche, pour une partie de l'électorat, être contre Jean-Marie Le Pen ne signifiait pas nécessairement voter pour Jacques Chirac. Or, comme cela a été dit, la valeur de l'indice augmente quand les électeurs sont amenés à prendre en compte plusieurs critères d'appréciation s'inscrivant dans des logiques différentes.

- Lisibilités comparées des différents référendums depuis $1958^{13}$

\begin{tabular}{|c|c|c|c|c|}
\hline & $\begin{array}{c}\text { Oui } \\
\text { (en proportion } \\
\text { des inscrits) }\end{array}$ & $\begin{array}{c}\text { Non } \\
\text { (en proportion } \\
\text { des inscrits) }\end{array}$ & $\begin{array}{c}\text { Abstentions, } \\
\text { blancs ou nuls } \\
\text { (en proportion } \\
\text { des inscrits) }\end{array}$ & $\begin{array}{c}\text { Indice de } \\
\text { lisibilité }\end{array}$ \\
\hline $\begin{array}{c}\text { Constitution de la Ve République } \\
\text { (1958) }\end{array}$ & $67,5 \%$ & $14,2 \%$ & $18,3 \%$ & 0,62 \\
\hline $\begin{array}{c}\text { Autodétermination de l'Algérie (1961) } \\
\text { Accords d'Évian sur l'indépendance de } \\
\text { l'Algérie (1962) }\end{array}$ & $53,6 \%$ & $17,9 \%$ & $28,5 \%$ & 0,83 \\
\hline $\begin{array}{c}\text { Élection du président de la République } \\
\text { au suffrage universel direct (1962) }\end{array}$ & $46,7 \%$ & $28,3 \%$ & $25 \%$ & 0,66 \\
\hline $\begin{array}{c}\text { Réforme du Sénat et régionalisation } \\
\text { (1969) }\end{array}$ & $37,1 \%$ & $40,8 \%$ & $22,1 \%$ & 0,56 \\
\hline $\begin{array}{c}\text { Élargissement de la Communauté } \\
\text { économique européenne (1972) }\end{array}$ & $36,4 \%$ & $16,9 \%$ & $46,7 \%$ & 1,31 \\
\hline $\begin{array}{c}\text { Statut de la Nouvelle Calédonie } \\
\text { (1988) }\end{array}$ & $26 \%$ & $6,5 \%$ & $67,5 \%$ & 2,16 \\
\hline $\begin{array}{c}\text { Ratification du traité de Maastricht sur } \\
\text { l'Union européenne (1992) }\end{array}$ & $34,4 \%$ & $33 \%$ & $32,6 \%$ & 0,84 \\
\hline $\begin{array}{c}\text { Quinquennat } \\
\text { (2000) }\end{array}$ & $30,7 \%$ & $37 \%$ & $32,3 \%$ & 2,39 \\
\hline $\begin{array}{c}\text { Constitution européenne } \\
\text { (2005) }\end{array}$ & $6,8 \%$ & $74,7 \%$ & 0,84 \\
\hline
\end{tabular}

On constate que les référendums ont, pour les électeurs, une lisibilité généralement plus faible que les élections présidentielles.

$\mathrm{Au}$ vu de ces tableaux, j'aurais tendance à considérer qu'un indice de lisibilité inférieur à 0,5 correspond à une assez bonne lisibilité ; entre 0,5 et 1 , à une lisibilité passable, entre 1 et 2 à une lisibilité médiocre ; au dessus de 2 , à une lisibilité très faible.

\section{I.2. LES DÉTERMINANTS DE L’ABSTENTION}

Dans notre représentation, les électeurs qui se considèrent suffisamment proches d'une des options qui leur sont proposées, votent. Les autres s'abstiennent. Ainsi analysés, les votes blancs ou nuls sont assimilés à une forme d'abstention. Le taux d'abstention est

\footnotetext{
${ }^{13}$ Les Indices de lisibilité figurant dans ce tableau correspondent aux moyennes calculées sur l'ensemble des circonscriptions. Ils sont légèrement supérieurs à ceux que l'on obtient en ne prenant en compte que les départements de la France métropolitaine, comme nous l'avons fait pour la plupart des exemples développés dans cet article.
} 
ainsi défini comme la proportion d'électeurs qui ne peuvent pas ou ne veulent pas choisir entre les candidats en présence.

Le modèle théorique établit une relation entre le taux d'abstention $\mathrm{N}_{=}$, la lisibilité d'un scrutin $\mathrm{I}_{\mathrm{AB}}$, et l'écart de score entre les candidats, $[\mathrm{N}(\mathrm{A})-\mathrm{N}(\mathrm{B})]$;

Sous sa forme la plus générale, cette relation s'exprime approximativement par :

$$
\mathrm{N}_{=}=1-[\mathrm{N}(\mathrm{A})+\mathrm{N}(\mathrm{B})] \cong \alpha-\beta \cdot[\mathrm{N}(\mathrm{A})-\mathrm{N}(\mathrm{B})]^{2}
$$

$\alpha$ et $\beta$ sont deux constantes, fonctions monotones croissantes de $\mathrm{I}_{\mathrm{AB}}{ }^{14}$.

L'abstention dépendrait ainsi de deux facteurs : la lisibilité des enjeux du scrutin (constante pour une élection donnée), et l'écart de score entre les candidats (variable selon les circonscriptions).

On en déduit un certain nombre de conséquences théoriques, qui correspondront d'autant plus à la réalité observée, que l'électorat étudié est homogène :

- L'abstention est à son maximum quand les deux candidats ont un poids électoral identique. Elle est alors égale, à un facteur près, à l'indice de lisibilité. De ce point de vue, celui-ci constitue bien, comme nous l'avons dit, un indicateur de la tendance à l'abstention pour une élection donnée.

- D'une circonscription à l'autre, le taux d'abstention diminue, à partir de ce maximum, en fonction de l'écart de score entre les candidats ${ }^{15}$. En d'autres termes, des électeurs, indécis quand les deux candidats sont au coude à coude, «volent au secours de la victoire » quand celle-ci semble acquise pour l'un des candidats.

- Par rapport à un état donné de l'opinion (défini par l'ensemble des proximités individuelles), l'abstention augmente quand l'un des candidats se rapproche de son adversaire ou quand il se rapproche de l'opinion moyenne : En d'autres termes, en cherchant à convaincre les indécis, il rend indécis une partie de ceux qui envisageaient initialement de voter, y compris pour lui.

\section{- L'exemple du second tour de l'élection présidentielle de 2002}

Parmi les scrutins récents, le second tour de l'élection présidentielle de 2002 est celui dans lequel l'écart de score entre les candidats étant le plus marqué, la relation entre le taux d'abstention et le carré de l'écart de score des deux candidats, prévue par le modèle théorique, apparaît le plus nettement.

Le coefficient de variabilité de 0,096 correspond à un électorat relativement homogène ${ }^{16}$.

\footnotetext{
${ }^{14}$ Les calculs développés en annexe montrent que dans les configurations communément observées $(\mathrm{I}<0,9), \alpha \cong 0,4 . \mathrm{I}_{\mathrm{AB}}$ et $\beta \cong 0,8 . \alpha$.

${ }^{15}$ En fait, les données recueillies montrent que l'ampleur de ce phénomène n'est significative que lorsque l'écart de score entre les candidats est important: d'une circonscription à l'autre, le taux d'abstention diminue comme le carré de cet écart. Quand cet écart est faible, le phénomène est donc relativement peu marqué, et il peut être masqué, par exemple par les hétérogénéités de l'électorat.

${ }^{16}$ Nous verrons que, en fait, les électorats de J. Chirac et de J.-M. Le Pen étaient sensiblement différents mais que, celui de J.-M. Le Pen étant fortement minoritaire, l'impact de cette différence sur l'homogénéité de l'ensemble apparaît limité - contrairement à ce qu'on pourra observer en 2007 avec les électorats de S. Royal et N. Sarkozy.
} 


\begin{tabular}{|l|c|c|c|c|}
\hline \multicolumn{5}{|c|}{ France métropolitaine } \\
\hline & \% Chirac & \% Le Pen & Écart & $\begin{array}{c}\% \\
\text { Abstention }\end{array}$ \\
\cline { 2 - 5 } & 70,8 & 8,6 & 62,2 & 20,7 \\
\cline { 2 - 5 } Moyennes calculées sur des & 68,5 & 9,5 & 59 & 21,9 \\
\cline { 2 - 5 } $\begin{array}{l}\text { groupes de dix départements } \\
\text { classés en fonction de l'écart des } \\
\text { scores obtenus par les deux } \\
\text { candidats. }\end{array}$ & 66,7 & 11,2 & 55,5 & 22,1 \\
\cline { 2 - 5 } & 64,4 & 12,3 & 52,2 & 23,3 \\
\cline { 2 - 5 } & 63,3 & 13,8 & 49,5 & 22,9 \\
\cline { 2 - 5 } & 61,9 & 14,1 & 47,7 & 24 \\
\cline { 2 - 5 } & 60,3 & 15,7 & 44,6 & 24,1 \\
\cline { 2 - 5 } & 58,9 & 16,6 & 42,3 & 24,5 \\
\hline
\end{tabular}

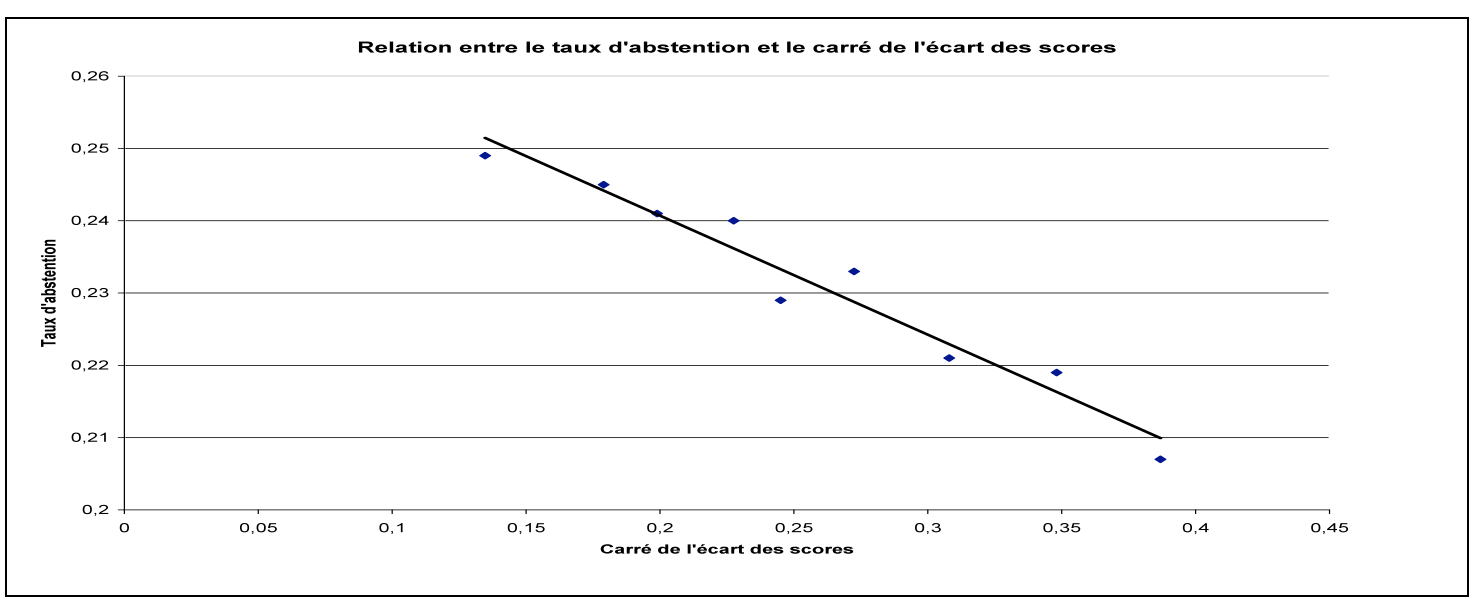

\section{I.3. ABSTENTION OBSERVÉE, ABSTENTION THÉORIQUE ET ABSTENTION STRUCTURELLE}

Dans la réalité, l'abstention observée ne coïncide pas avec la définition précédente. Il s'y superpose une autre forme d'abstention, «structurelle », variable d'une circonscription à l'autre : celle des électeurs inscrits qui ne votent pas, par indifférence vis-à-vis du jeu électoral, ou simplement parce qu'ils en sont matériellement empêchés (voyage, maladie...) $)^{17}$.

Lorsque l'électorat est homogène, cette abstention structurelle est, par définition, indépendante de la position relative des électeurs par rapport aux options qui leur sont proposées. Elle est donc sans incidence sur le résultat de l'élection. Elle n'affecte pas la représentation des choix électoraux, mais elle introduit un biais dans l'évaluation des paramètres qui leur sont associés : la valeur de l'indice de lisibilité, estimée d'après les résultats du scrutin, est plus élevée que sa valeur théorique ; l'électorat semble plus hétérogène qu'il ne l'est en réalitée ${ }^{18}$.

17 Parmi les électeurs inscrits qui ne votent pas, figurent les électeurs inscrits à tort sur des listes électorales non actualisées. Il n'est pas exclu que le fort taux d'abstention enregistré en Corse à chaque élection comporte une composante de cet ordre...

${ }^{18} \mathrm{Cf}$. Annexe. 
Lorsque les variations de l'abstention structurelle d'une circonscription à l'autre sont relativement importantes, elles peuvent ainsi masquer quelques uns des phénomènes que l'on cherche à mettre en évidence. C'est le cas notamment de la relation établie par le modèle théorique entre le taux d'abstention et la différence de score des candidats, quand cette différence est faible.

Un certain nombre de procédures statistiques permettent toutefois de contourner l'obstacle et de mettre évidence les tendances prévues. La plus simple consiste à regrouper les circonscriptions étudiées en fonction de la valeur de la caractéristique dont on cherche à évaluer l'influence, puis à travailler sur les valeurs moyennes obtenues.

Les calculs développés en annexe montrent que, si l'électorat est effectivement homogène, on doit pouvoir observer une relation linéaire de la même forme que celle qui correspond au modèle théorique : $\mathrm{N}=\cong \alpha-\beta$. $[\mathrm{N}(\mathrm{A})-\mathrm{N}(\mathrm{B})]^{2}$, entre le taux d'abstention observé et le carré de l'écart de score. Les cœfficients $\alpha$ et $\beta$ ainsi estimés permettent d'obtenir une évaluation de l'abstention théorique et de l'abstention structurelle.

C'est le cas par exemple des résultats du second tour de la présidentielle de 2002 : sur un taux moyen d'abstention de 23,7 \%, la part de l'abstention structurelle peut être évaluée selon cette procédure à 10,5\%, soit près de la moitié de l'abstention totale, l'autre moitié étant représentée par les électeurs n'ayant pas voulu choisir entre J. Chirac et J.-M. Le Pen.

Plus généralement, si le regroupement de circonscriptions en fonction d'un critère (par exemple en fonction de l'écart relatif des scores des candidats) fait apparaître une corrélation entre $\mathrm{N}_{=}$et $[\mathrm{N}(\mathrm{A}) \text { : - } \mathrm{N}(\mathrm{B})]^{2}$, on peut en déduire que l'électorat est homogène, selon ce critère. La valeur du coefficient de corrélation de l'ajustement linéaire obtenu, donne une mesure de l'homogénéité.

Pour le second tour de l'élection présidentielle de 2002, le coefficient de corrélation en question est de 0,85. Il n'est que de 0,43 pour le second tour de la présidentielle de 2007. Par rapport au choix qui leur était proposé, l'électorat apparaît plus homogène en 2002 qu'en 2007.

\section{II. ÉLECTION COMPORTANT DES ENJEUX MULTIPLES}

Le modèle de base concerne une consultation comportant un enjeu unique. Ce n'est pas le cas dans la réalité.

La plupart des élections comportent des enjeux multiples, dont la pondération caractérise « une lecture des enjeux ». Celle-ci varie selon les électeurs. La pondération moyenne détermine la lisibilité moyenne des enjeux et donc le taux d'abstention. Elle peut évoluer au cours de la campagne électorale. Elle est alors susceptible d'affecter le résultat du scrutin.

L'analyse développée en annexe, dont nous reprenons ici les principales conclusions, concerne une consultation opposant deux candidats et comportant deux enjeux principaux. Elle repose sur les propriétés des lois normales, et notamment sur le fait que toute combinaison linéaire de deux variables normales indépendantes, est une variable normale. Elle peut, dans des conditions très générales, être étendue à une combinaison d'enjeux en nombre quelconque. 


\section{II.1. IMPACT D’UNE VARIATION DE LA PONDÉRATION DES ENJEUX SUR LES RÉSULTATS D’UNE ÉLECTION}

La représentation graphique utilisée ici permet de mettre en évidence les possibilités (et les limites) d'une stratégie visant, pour un candidat, à faire évoluer la pondération des enjeux de l'élection dans un sens qui lui soit favorable.

Le point O figure l'opinion moyenne. Deux axes, Ox et Oy représentent les deux principaux enjeux, c'est-à-dire ce sur quoi s'opposent les deux candidats, A et B. (Ox pourrait par exemple correspondre à «plus ou moins de protection sociale », Oy à «plus ou moins de politique sécuritaire »). Sur chacun de ces axes, deux points : $A_{x}$, $\mathrm{B}_{\mathrm{x}} ; \mathrm{A}_{\mathrm{y}}, \mathrm{B}_{\mathrm{y}}$ figurent les positions moyennes des deux candidats.

Dans l'exemple ci-dessous, A l'emporterait si l'élection se jouait sur l'axe Ox : $A_{x}$ est en effet plus proche de $\mathrm{O}$ que $\mathrm{B}_{\mathrm{x}}$. En revanche, si l'élection se jouait sur l'enjeu représenté par l'axe Oy, $\mathrm{B}$ l'emporterait, $\mathrm{B}_{\mathrm{y}}$ étant plus proche de $\mathrm{O}$ que $\mathrm{A}_{\mathrm{y}}$. Les deux couples $\left\{\mathrm{A}_{\mathrm{x}}, \mathrm{A}_{\mathrm{y}}\right\}$ et $\left\{\mathrm{B}_{\mathrm{x}}, \mathrm{B}_{\mathrm{y}}\right\}$ définissent les points $\mathrm{A}$ et $\mathrm{B}$, correspondant au positionnement de $\mathrm{A}$ et $\mathrm{B}$.

L'axe $O z$ correspond à une pondération des deux enjeux. Le résultat du scrutin sera déterminé par les positions relatives de $A_{z}$ et $B_{z}$, projections de A et $B$ sur cet axe.

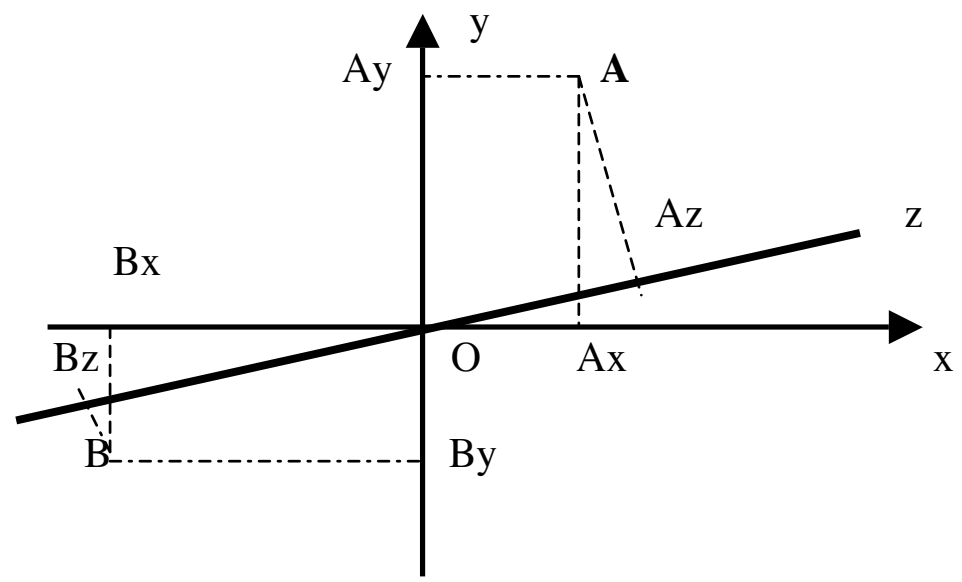

On peut poursuivre l'analyse en constatant qu'il existe deux axes, $O z_{1}$ et $O z_{2}$, sur lesquels les deux candidats sont à égale distance de l'opinion moyenne : $O A_{z}=O B_{z}$.

Ces deux axes correspondent à deux configurations dans lesquelles $\mathrm{A}$ et $\mathrm{B}$ obtiennent des scores identiques.

- Dans la première, les électeurs ne perçoivent globalement aucune différence entre les candidats. Une telle configuration est figurée par $\mathrm{Oz}_{1}$, axe perpendiculaire à $\mathrm{AB}$ : $\mathrm{OA}_{\mathrm{z} 1}$ et $\mathrm{OB}_{\mathrm{z} 1}$ sont confondus.

- Dans la seconde en revanche, les deux candidats sont perçus comme nettement différents. Cette configuration est figurée par $\mathrm{Oz}_{2}: \mathrm{OA}_{\mathrm{z} 2}$ et $\mathrm{OB}_{\mathrm{z} 2}$ sont symétriques ${ }^{19}$.

\footnotetext{
${ }^{19}$ On vérifiera que la position de $\mathrm{Oz}_{2}$ peut être déterminée de la manière suivante : on part des deux cercles de diamètre $\mathrm{OA}$ et $\mathrm{OB}$ : : $\mathrm{A}_{\mathrm{z2}}$ se situe à l'intersection du premier de ces cercles et du symétrique du second ; $\mathrm{B}_{z 2}$, à l'intersection du second de ces cercles et du symétrique du premier. $\mathrm{C}$ étant le milieu de $\mathrm{AB}, \mathrm{Oz}_{2}$ est perpendiculaire à $\mathrm{OC}$.
} 
Elle correspond à une situation de bipolarisation, dans laquelle l'électorat s'organise en deux blocs opposés de poids électoral identique.

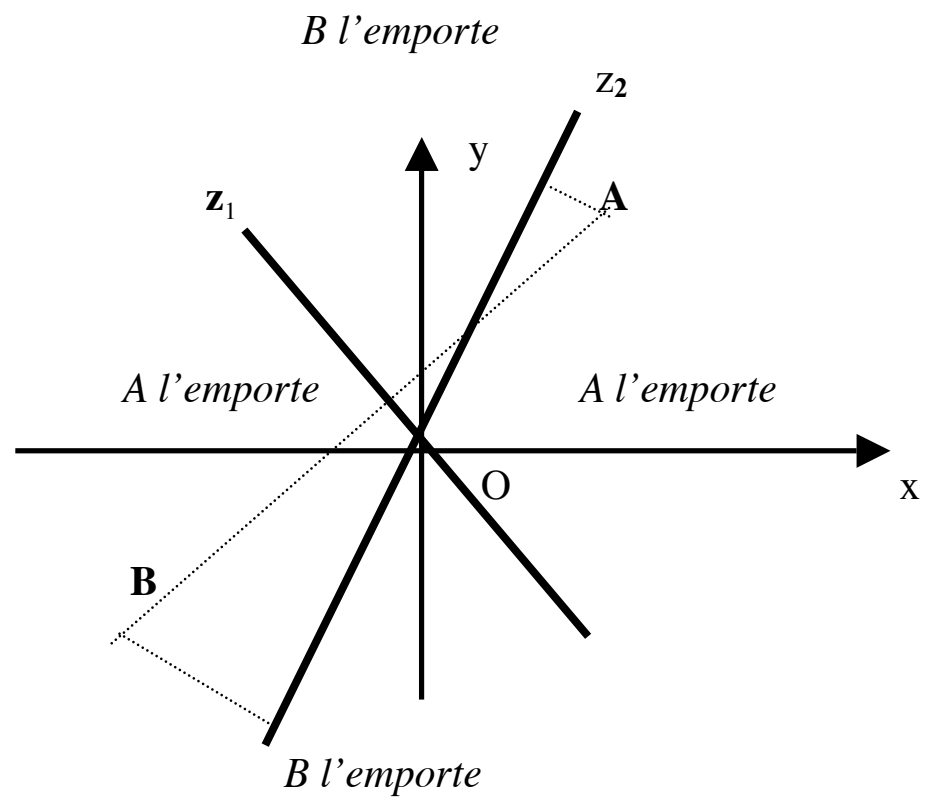

Les axes $\mathrm{Oz}_{1}$ et $\mathrm{Oz}_{2}$ délimitent les zones correspondant aux pondérations permettant soit à $\mathrm{A}$, soit à $\mathrm{B}$ de l'emporter. On en déduit que, théoriquement, quelle que soit la majorité pour un enjeu donné, la prise en compte d'un second enjeu peut entraîner un renversement de tendance.

Dans le même temps, comme nous le montrons en annexe, la prise en compte d'un enjeu supplémentaire réduit le plus souvent la lisibilité du scrutin, et donc accroît la propension à l'abstention. On montre ainsi, par exemple, que si les électeurs doivent prendre en compte deux enjeux indépendants de lisibilité comparable et auxquels ils accordent la même importance, l'abstention sera supérieure de plus de $40 \%$ à ce qu'elle aurait été avec un enjeu unique.

Nous verrons dans un prochain article que l'intervention d'un troisième candidat, dans une élection opposant à l'origine deux candidats a un effet similaire : elle réduit la lisibilité des enjeux et accroît le taux d'abstention.

\section{II.2. APPLICATION À L'ANALYSE DES STRATÉGIES DES CANDIDATS}

D'après ce qui vient d'être exposé, le résultat d'une élection dans laquelle deux candidats sont opposés l'un à l'autre, apparaît déterminé par deux paramètres : leur positionnement relatif par rapport à l'opinion moyenne et la pondération des enjeux.

Que peut-on en déduire sur les stratégies des candidats?

Schématiquement, à partir d'une situation donnée, chaque candidat va tenter d'ajuster son positionnement et de faire évoluer la pondération des enjeux, en choisissant de privilégier l'une ou l'autre de ces stratégies : faire le plein des voix de son camp en mettant l'accent sur ce qui l'oppose le plus à son adversaire aux yeux de ses partisans, ou se rapprocher de l'opinion moyenne pour élargir son potentiel de voix. 
Il est généralement admis que, si l'élection est serrée, la seconde option s'impose naturellement. Elle paraît à première vue d'autant plus légitime que, si l'un des candidats l'adopte, il semble difficile à l'autre de ne pas le suivre : comment imaginer qu'un candidat dans une position incertaine abandonne à son adversaire le vote potentiel des indécis?

En réalité, les choses ne sont pas aussi simples : en cherchant à élargir son électorat en direction des indécis, un candidat rend indécis une partie de ses électeurs potentiels. Il est en effet conduit à se rapprocher du centre ou à se rapprocher de son adversaire - de manière à prendre en compte les préoccupations de ceux qui, au départ, n'étaient pas prêts à voter pour lui. Ce qui, dans tous les cas, aboutit mécaniquement à réduire l'expression de ce qui distingue les deux adversaires sur les enjeux du scrutin. Le débat initial dans lequel une partie de l'électorat a du mal à s'impliquer, se déplace ainsi au profit de questions sur lesquelles tout un chacun peut avoir une opinion, comme celles qui concernent la personnalité des candidats.

Un tel processus a une première conséquence. Il tend à accroître le taux d'abstention puisqu'il a pour effet de réduire la lisibilité des enjeux : des électeurs au départ potentiellement acquis à l'un ou l'autre des candidats, mais sans enthousiasme, avec des réserves sur tel ou tel aspect du programme ou de sa personnalité, choisiront finalement de s'abstenir - sans que cette abstention soit compensée par les voix acquises auprès des indécis. Une campagne dans laquelle chacun s'efforce de convaincre les indécis en multipliant les prises de position sur les questions les plus diverses, ne peut - éventuellement - être efficace qu'auprès d'un électorat fortement hétérogène, segmenté autour de revendications catégorielles ou communautaires. Ce qui est de moins en moins le cas dans la plupart des pays.

La deuxième conséquence concerne l'issue du scrutin. Dans une configuration où les deux candidats ont un poids électoral comparable, une faible variation dans la représentation des enjeux du vote ou dans la perception de la personnalité des candidats, au sein de la fraction la plus hésitante de l'électorat, suffit alors à décider du résultat. En d'autres termes, plus les candidats font campagne au centre pour convaincre les indécis, plus le résultat du vote est incertain, et plus il se joue sur «des enjeux subalternes » parmi les électeurs les moins politisés. 
La notion de lisibilité appliquée aux enjeux d'une élection

Nous avons défini le défaut de lisibilité d'un scrutin opposant deux candidats, comme la somme des proximités moyennes des deux candidats.

Appliqué aux enjeux d'une élection, le terme de lisibilité renvoie aux travaux menés, notamment dans les pays anglo-saxons, autour de la question de la compréhension d'un texte lorsque celle-ci dépend de l'implication du destinataire ou de sa connaissance du sujet. Ces études sont loin d'être purement théoriques puisqu'elles ont débouché dans plus de la moitié des États américains sur des normes légales, concernant aussi bien la clarté des formulaires de "consentement éclairé » soumis aux patients avant une opération, que les documents électoraux.

Certains travaux, comme ceux que Dale et Chall ${ }^{20}$ ont consacrés aux processus participant à la réception et la compréhension d'un discours, conduisent ainsi à s'intéresser à la signification donnée par les électeurs aux mots censés traduire leurs préoccupations et aux conditions de leur utilisation par les hommes politiques. En utilisant des termes similaires pour désigner des contenus politiques différents (la construction de l'Europe ; le progrès social; la défense de l'emploi...) les candidats introduisent, selon la formulation que nous avons utilisée, « des alternatives floues » contribuant à l'indécision des électeurs et donc à l'abstention ${ }^{21}$.

D'autres recherches, en particulier celles qui s'inscrivent dans la mouvance du modèle construction-intégration ${ }^{22}$, associent la compréhension d'un discours à la capacité de son destinataire de le décomposer en une suite de propositions simples dont le sens lui est évident. Dans le cas d'une élection, une telle représentation renvoie à l'idée selon laquelle un électeur appréhenderait les choix qui lui sont proposés à partir d'un ensemble d'alternatives élémentaires. On pourrait ainsi considérer comme l'un des motifs d'abstention, l'absence d'un système de représentation adéquat permettant de classer ou d'intégrer les différentes alternatives les unes par rapport aux autres.

Ces deux voies d'approches sont naturellement complémentaires ${ }^{23}$. Elles associent le défaut de lisibilité d'un scrutin à la difficulté de l'électeur, soit de se prononcer sur certaines des alternatives conditionnant son vote, soit de rendre ses choix cohérents entre eux. En d'autres termes, plus les critères à prendre en compte sont nombreux et indépendants les uns des autres, moins les enjeux sont lisibles.

\section{III. ÉLECTORAT NON HOMOGÈNE}

Le modèle de référence correspond à un électorat parfaitement homogène dans un scrutin opposant deux candidats : l'indice de lisibilité, c'est à dire la somme de leurs proximités moyennes, est alors constant. Dans la réalité, un électorat n'est jamais

\footnotetext{
${ }^{20}$ J.S. Chall et E. Dale, Reability revisited, Cambridge, Brookline books, 1995.

${ }^{21}$ Le fait qu'un terme soit connu n'indique pas qu'il fasse sens pour le destinataire. Ainsi, d'après une étude du CERC, seuls $21 \%$ des Français seraient capables de préciser le sens de mots comme expansion et productivité (Cité par B. Labasse, "La lisibilité rédactionnelle: fondements et perspectives", Communication et langage, $\mathrm{n}^{\circ} 131$.

${ }^{22}$ W. Kintsch, Compréhension, a paradigm for cognition, Cambridge, Cambridge University Press, 1998.

${ }^{23}$ Les deux approches recoupent la distinction traditionnelle en linguistique, entre les processus de compréhension de bas niveau (bottom-up) qui dépendent uniquement du décodage du texte, et les processus de haut niveau (top-down) qui font intervenir les connaissances et l'implication du sujet.
} 
parfaitement homogène. L'indice de lisibilité varie plus ou moins d'une circonscription à l'autre.

L'analyse développée en annexe montre qu'un électorat non homogène peut s'analyser comme composé de groupes homogènes distincts, ayant des lectures différentes des enjeux et présents dans les différentes circonscriptions dans des proportions variables. En pratique, une partition en deux groupes est généralement suffisante pour rendre compte de l'essentiel de l'hétérogénéité observée.

On montre que, dans ce cas, l'hétérogénéité de l'électorat, mesurée par la variabilité de l'indice de lisibilité, est proportionnelle à la différence des indices de lisibilité des deux groupes et à la variabilité de leur poids relatif. En d'autres termes lorsque le poids relatif des deux groupes ne varie pas trop d'une circonscription à l'autre, et/ou lorsque leurs lectures des enjeux sont relativement proches, le coefficient d'hétérogénéité est faible : l'électorat peut être considéré comme "quasi-homogène ».

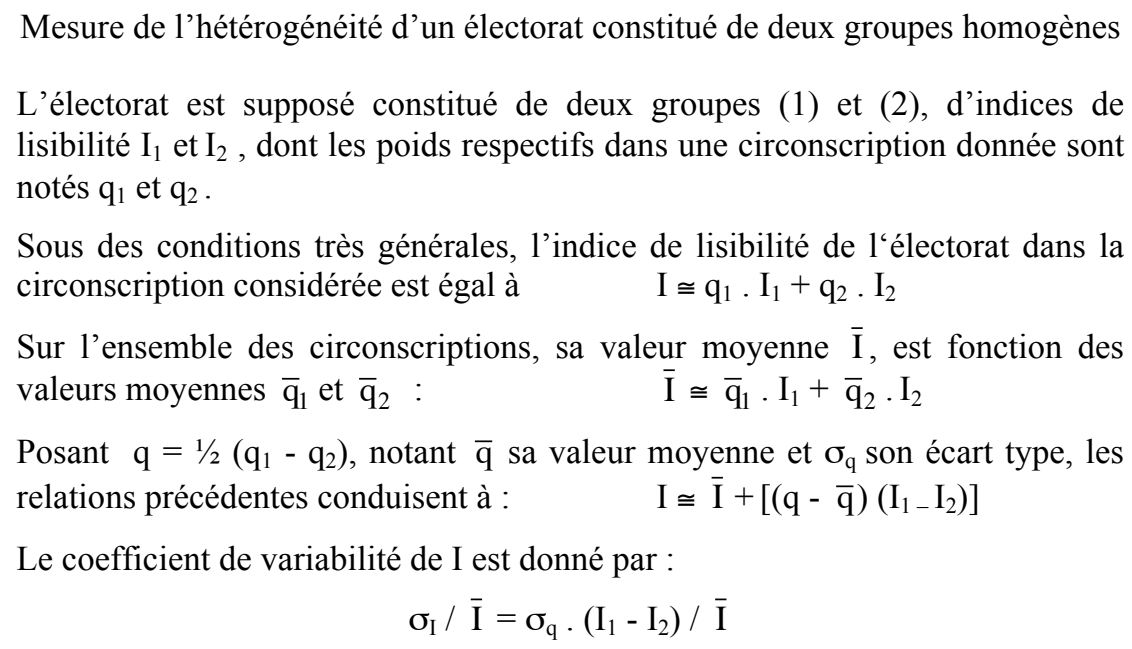

Si chacun des groupes composants présente les propriétés d'un électorat homogène, la plupart de ces propriétés se retrouvent au niveau de l'électorat pris dans son ensemble. C'est le cas notamment de la relation entre l'abstention et la lisibilité du scrutin.

Au sein de chaque groupe, l'abstention est fonction de la lecture des enjeux. Elle tend à augmenter quand l'indice de lisibilité qui lui est associé, augmente - notamment quand les candidats se rapprochent l'un de l'autre ou se rapprochent du centre. Il en est donc de même pour un électorat formé de la réunion de ces groupes.

\section{III.1. L'HÉTÉROGÉNÉITÉ D’UN ÉLECTORAT COMME ÉLÉMENT D’ANALYSE DU RÉSULTAT D'UN SCRUTIN}

Nous avons vu que la propension à l'abstention est d'autant plus forte que la lisibilité des enjeux est faible. On en déduit que, dans un électorat composé de deux groupes ayant des perceptions différentes des enjeux, le groupe ayant la lecture la plus simple est celui qui se mobilise le plus. 
Si ce groupe vote majoritairement pour A, le second groupe votant majoritairement pour B, la participation plus forte des partisans de A peut suffire à lui assurer la victoire.

Les élections régionales françaises de 2004 apparaissent de ce point de vue, exemplaires.

Pour l'opposition, ces élections étaient présentées comme l'occasion d'un vote sanction à dimension nationale, contre la politique du gouvernement. Le gouvernement, au contraire, affirmait qu'il s'agissait d'un enjeu strictement local : le choix d'un exécutif régional. Les résultats du scrutin ont montré que la majorité des électeurs ont privilégié la composante nationale, et une minorité, variable selon les régions, la composante régionale.

Le choix était relativement simple et la lisibilité plus forte pour les électeurs dont le vote se référait principalement à l'enjeu national. Il l'était un peu moins pour les électeurs qui se déterminaient essentiellement en fonction de l'enjeu local. Selon l'analyse qui vient d'être proposée, les premiers se sont relativement plus mobilisés que les seconds : plus la proportion d'électeurs dans ce cas est importante, moins l'indice de lisibilité doit être élevé.

Il est intéressant d'observer l'indice de lisibilité dans les quatre régions de la France métropolitaine où seules deux listes restaient en présence au second tour (Pays de Loire, Bretagne, Auvergne, et Limousin.

L'indice de lisibilité apparaît légèrement plus élevé dans les Pays de Loire qu'en Bretagne ou qu'en Auvergne. On peut en déduire que la composante régionale a pesé plus dans le premier cas que dans les deux autres. Le Limousin, où l'on observe des différences sensibles d'un département à l'autre, représente une configuration intermédiaire.

\begin{tabular}{|c|c|c|c|}
\hline \multicolumn{4}{|c|}{ Valeur de l'indice de lisibilité } \\
\hline Pays de Loire & Bretagne & Auvergne & Limousin \\
\hline 1,04 & 0,92 & 0,92 & 0,98 \\
\hline
\end{tabular}

On peut affiner l'analyse en considérant que la lecture des enjeux était plus difficile encore pour ceux qui avaient à arbitrer entre des options apparemment contradictoires, par exemple voter localement pour la liste présentée par l'UMP parce que celle-ci leur paraissait plus à même de défendre leurs intérêts, tout en désapprouvant l'action du gouvernement. Il est logique d'en déduire que l'indice de lisibilité sera plus élevé que la moyenne dans les circonscriptions où une proportion importante d'électeurs était dans cette position - et inversement, qu'il sera plus faible que la moyenne là où, pour des raisons locales, les électeurs étaient majoritairement acquis soit à la gauche, soit à la droite.

C'est effectivement ce qu'on observe dans les villes dont les têtes de liste étaient par ailleurs les élus: François Fillon (UMP) à Sablé et Jacques Auxiette (PS) à La Roche-sur-Yon, dans les Pays de Loire ; Josselin de Rohan (UMP) à Josselin et JeanYves le Drian (PS) à Vannes, en Bretagne.

À Josselin, la population a voté très majoritairement pour les régionales comme elle vote pour son maire : l'indice de lisibilité y est plus faible que la moyenne de la 
M. FANSTEN

région. À Sablé, au contraire, même si François Fillon reste majoritaire, une partie de ses électeurs s'est abstenue ou a voté pour la liste de gauche : l'indice de lisibilité y est plus élevé que la moyenne de la région. À Vannes comme à La Roche-sur-Yon, l'indice de lisibilité est proche de la moyenne de la région concernée : les électeurs ont voté en fonction des mêmes critères que dans le reste de la région ; la personnalité du maire n'a pas eu d'effet d'entraînement supplémentaire :

\begin{tabular}{|c|c|c|c|c|c|c|}
\hline \multicolumn{5}{|c|}{ Valeur de l'indice de lisibilé } \\
\hline \multicolumn{2}{|c|}{ Bretagne } & & \multicolumn{3}{c|}{ Pays de Loire } \\
\cline { 1 - 3 } \cline { 5 - 7 } Ensemble de la région & Vannes & Josselin & & Ensemble de la région & La Roche/Yon & Sablé \\
\hline 0,92 & 0,95 & 0,74 & & 1,04 & 1,01 & 1,14 \\
\hline
\end{tabular}

\section{III.2. PARTITION D’UN ÉLECTORAT HÉTÉROGÈNE EN DEUX GROUPES HOMOGÈNES}

Dans le cas des élections régionales de 2004, la nature de l'hétérogénéité est relativement simple à identifier: le contexte de l'élection fournit une hypothèse de travail qu'il est facile de confronter aux résultats du scrutin.

Ce n'est pas toujours le cas. On doit procéder en deux temps : tenter d'identifier $a$ priori les deux groupes, puis chercher à déterminer si, effectivement, la représentation obtenue explique (au sens statistique) les variations observées de l'indice de lisibilité. C'est-à-dire s'il existe, d'une circonscription à l'autre, une corrélation significative entre ces variations et le poids relatif des deux groupes. Il est alors possible d'obtenir une évaluation des indices de lisibilité, correspondant à chacune des deux composantes de l'électorat, et permettant de prendre la mesure de l'impact de cette hétérogénéité sur les résultats du scrutin.

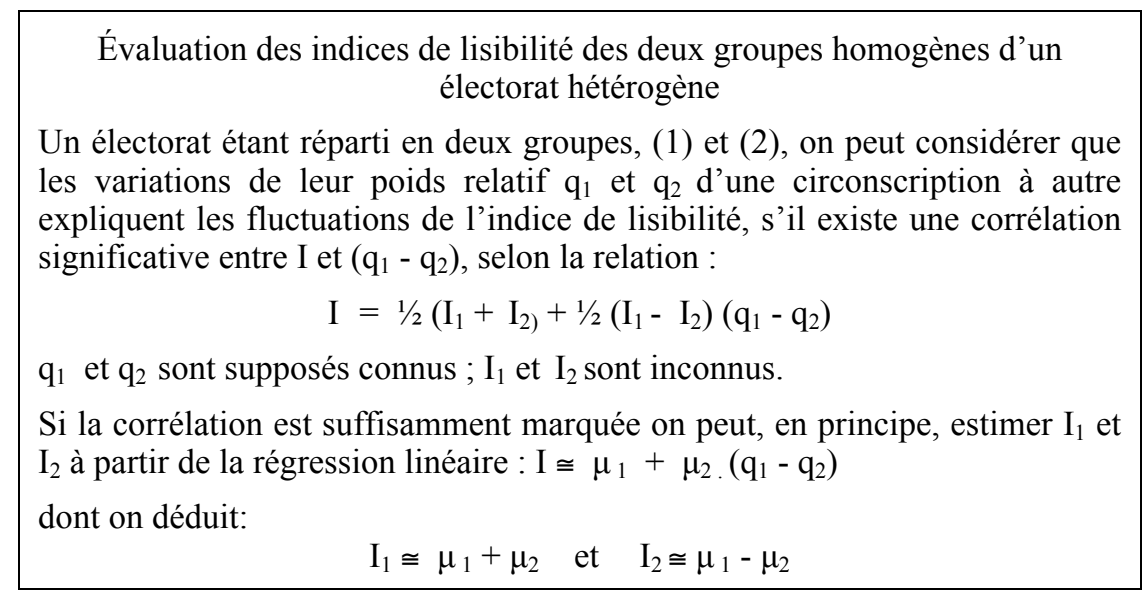

En général, il n'est pas facile de d'identifier a priori les deux groupes en question. Sauf dans deux configurations :

1. La première est celle où - comme en 2004 - la partition en deux groupes recoupe l'électorat des deux candidats : les électeurs du groupe (1) votent majoritairement (A), les électeurs du groupe (2) votent majoritairement B. Dans ce cas, selon la formule précédente, il doit y avoir une corrélation significative entre l'indice de lisibilité et la différence des scores relatifs des candidats. 
2. La seconde configuration est celle où la partition en deux groupes ne recoupe pas l'électorat des deux candidats, mais se retrouve d'une élection à l'autre, pour des consultations de même nature. Dans ce cas, on doit pouvoir observer une corrélation significative entre l'indice de lisibilité pour une élection donnée, et un indicateur fonction du poids relatif des deux groupes, obtenu dans une élection antérieure : score des candidats, taux d'abstention..., sans qu'il soit nécessaire d'identifier plus précisément les groupes concernés.

- L'exemple du second tour de l'élection présidentielle de 2007

Le second tour de l'élection présidentielle de 2007 fournit un exemple de la première configuration: les tests d'homogénéité appliqués aux résultats sont peu concluants, notamment ceux qui concernent la corrélation supposée entre le taux d'abstention et le carré de l'écart de score entre N. Sarkozy et S. Royal.

En revanche, on observe une corrélation très nette $(r=0,84)$ entre l'écart des scores des candidats et l'indice de lisibilité, d'autant plus marquée que cet écart est important. L'indice de lisibilité diminue quand le score de Ségolène Royal augmente. En d'autres termes, la lisibilité des enjeux est plus forte parmi les partisans de cette dernière - ou les opposants à Nicolas Sarkozy - qui se sont donc relativement plus mobilisés que le camp adverse, même si cette mobilisation a été insuffisante pour assurer la victoire de leur candidate ${ }^{24}$.

\begin{tabular}{|c|c|c|c|c|c|}
\hline \multicolumn{6}{|c|}{ Élection présidentielle de 2007} \\
\hline \multirow{10}{*}{$\begin{array}{l}\text { Moyennes calculées sur des } \\
\text { groupes de dix départements } \\
\text { classés en fonction des scores } \\
\text { obtenus par les deux candidats }\end{array}$} & $\begin{array}{c}\% \\
\text { Abstention } \\
\end{array}$ & $\%$ Sarkozy & $\%$ Royal & Écart & $\begin{array}{l}\text { Indice de } \\
\text { lisibilité }\end{array}$ \\
\hline & 16,7 & 44,1 & 55,9 & $-11,7$ & 0,42 \\
\hline & 17 & 47,2 & 52,8 & $-5,7$ & 0,43 \\
\hline & 18,1 & 49,1 & 50,9 & $-1,8$ & 0,46 \\
\hline & 18,6 & 51,2 & 48,8 & 2,4 & 0,47 \\
\hline & 18,1 & 52,9 & 47,1 & 5,8 & 0,46 \\
\hline & 18,1 & 54,6 & 45,4 & 9,1 & 0,46 \\
\hline & 17,6 & 55,9 & 44,1 & 11,8 & 0,45 \\
\hline & 18,8 & 57,2 & 42,8 & 14,5 & 0,48 \\
\hline & 19,2 & 59,9 & 40,1 & 19,7 & 0,5 \\
\hline
\end{tabular}

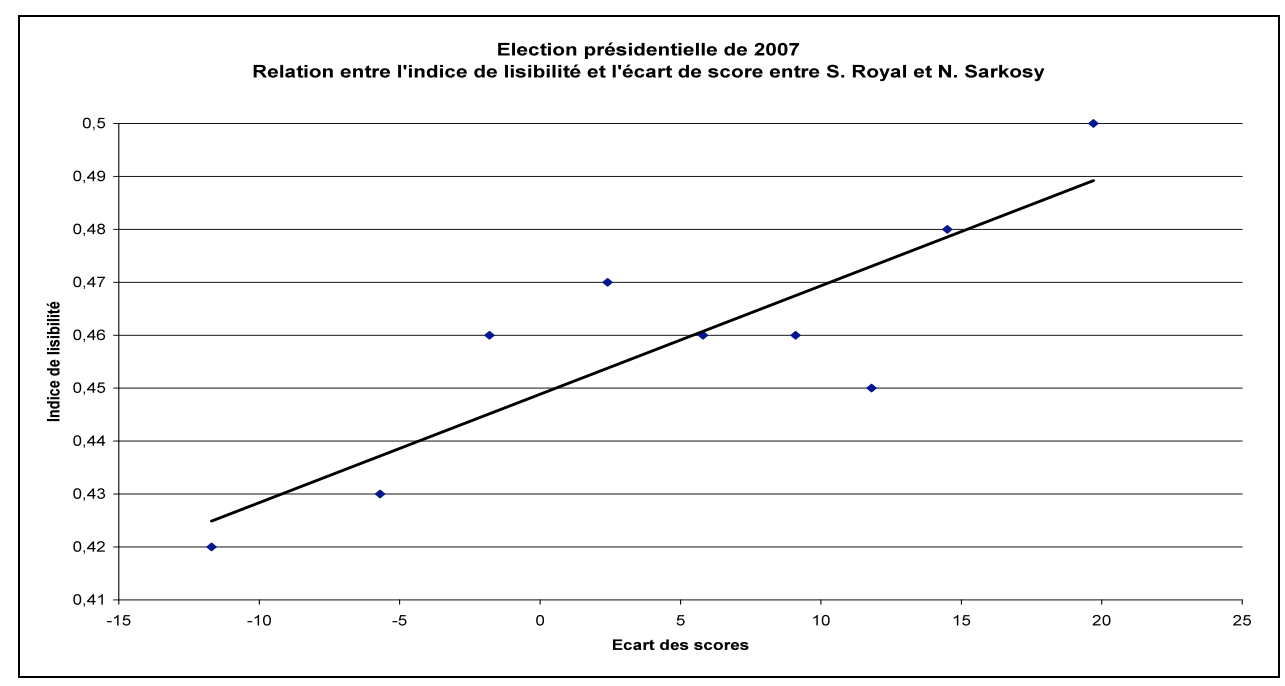

\footnotetext{
${ }^{24}$ Détail en Annexe.
} 
- L'exemple du référendum de 2005 sur le projet de constitution européenne

Le référendum de 2005 sur le projet de Constitution européenne fournit un exemple de la seconde configuration. Là encore, l'électorat n'apparaît pas très homogène. Mais, contrairement à ce qui vient être décrit à propos l'élection présidentielle de 2007, il n'y a pratiquement pas de corrélation entre l'indice de lisibilité et l'écart de score des options en présence. En revanche on observe une corrélation très nette entre l'indice de lisibilité du référendum de 2005 et le taux d'abstention des élections européennes de $2004^{25}$.

L'électorat de 2005 se décomposerait ainsi en deux groupes : ceux qui sont très concernés par les questions européennes (ils avaient voté en 2004) et ceux qui sont moins concernés (ils s'étaient abstenus en 2004).

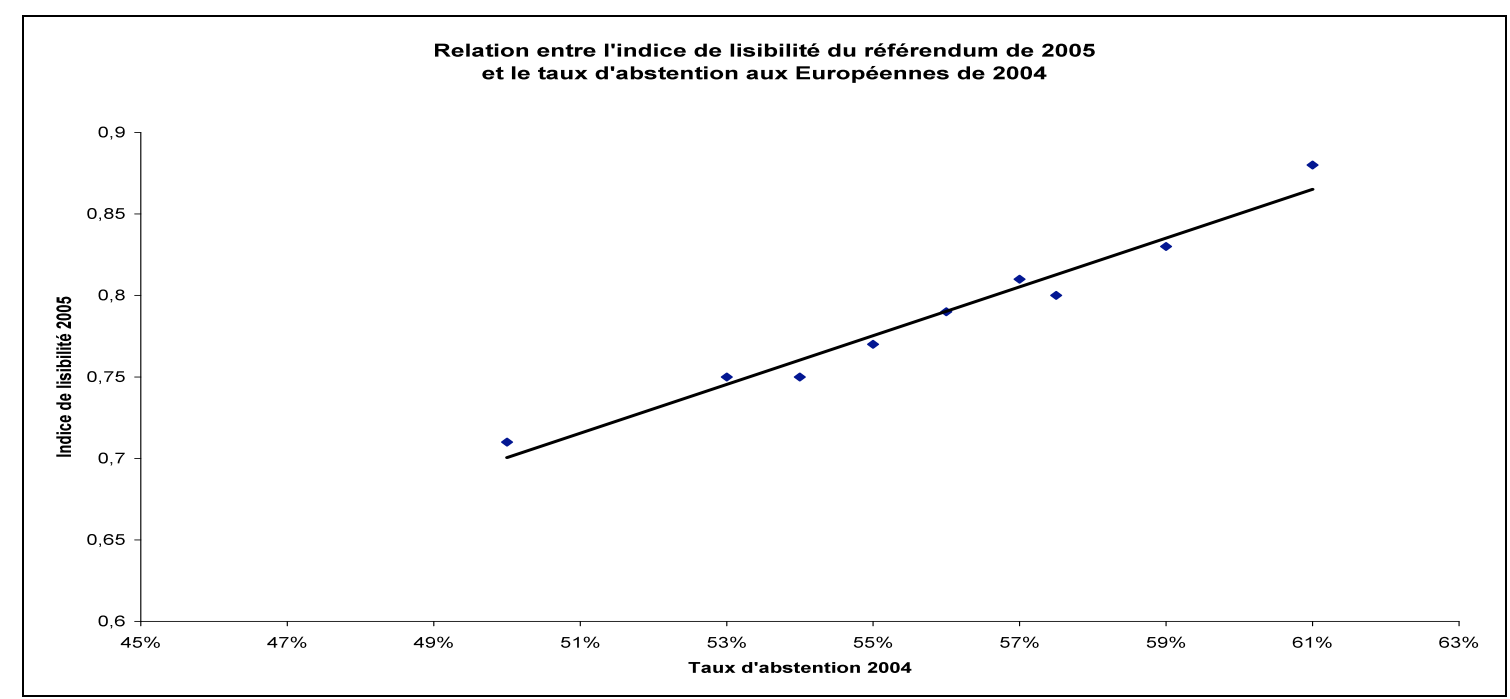

La faible participation de 2004 était en partie due aux réticences, majoritaires dans l'opinion, vis-à-vis de tel ou tel aspect de la construction européenne : celles des eurosceptiques face à l'élargissement de l'Union ou celles des électeurs opposés à l'orientation libérale de cet élargissement. Une telle conjonction n'avait pas seulement entraîné un fort taux d'abstention, elle avait aussi abouti, paradoxalement, à envoyer à Strasbourg une majorité favorable à la fois à l'élargissement et à son orientation libérale. Le phénomène inverse à joué en 2005 : plus de la moitié des électeurs qui s'étaient abstenus en 2004, ont voté en 2005. La représentation utilisée montre qu'ils ont voté majoritairement non $^{26}$.On peut penser que les raisons pour lesquelles ils ont voté non sont pour une large part les même que celles pour lesquelles ils s'étaient abstenus en 2004.

\footnotetext{
${ }^{25} \mathrm{Cf}$. Annexe : l'utilisation de l'indice de lisibilité dans la comparaison des résultats de deux élections.

${ }^{26}$ Ainsi, en Ile-de-France dans les trois départements qui ont voté très majoritairement oui (Paris, Hautsde-Seine, Yvelines) le taux d'abstention moyen avait été de l'ordre de $50 \%$ en 2004. Il avait été d'environ $60 \%$ dans les trois départements qui ont voté très majoritairement non (Val-d'Oise, Seine et Marne-et-Seine, Seine-Saint-Denis).
} 


\section{La notion d'électorat homogène}

La notion d'électorat homogène introduite dans le modèle de référence, se réfère à une population d'électeurs ayant sensiblement la même lecture des enjeu $^{27}$. Chaque électeur effectue alors son choix (voter pour l'un des candidats ou s'abstenir), en fonction de « ses données personnelles ", c'est-àdire de ce qui lui permet de se situer par rapport aux candidats en présence.

Plusieurs propriétés sont attachées à une telle représentation : lorsqu'une population est homogène, toutes les opinions sont représentées mais avec une fréquence décroissante au fur et à mesure qu'on s'éloigne de l'opinion médiane ; quand deux options sont en présence il n'y a pas de coupure nette entre ceux qui choisissent l'une et ceux qui choisissent l'autre, mais une différence de degré dans l'acceptation ou le refus, avec la zone intermédiaire de ceux hésitent entre l'une et l'autre; ou encore, un mouvement d'opinion sur une partie de l'électorat affecte l'électorat dans son ensemble.

L'homogénéité du comportement des électeurs ne ferait alors que traduire leur appartenance à une communauté, lorsque celle-ci implique des valeurs, des normes et des représentations communes.

Par opposition, la non-homogénéité correspondrait à un électorat constitué de plusieurs groupes ayant des lectures différentes des enjeux du scrutin et ne votant pas en fonction des mêmes critères.

Ces différences peuvent correspondre à des clivages liés à des caractéristiques sociales, économiques ou culturelles des électeurs (donc existant en dehors et indépendamment de la consultation concernée), ou être induites par la campagne électorale ou son contexte. Les diverses interprétations ne sont naturellement pas incompatibles ${ }^{28}$.

Quelle que soit leur origine, les différences de lecture des enjeux ont une conséquence : elles induisent des propensions à l'abstention plus ou moins fortes. Les groupes d'électeurs ayant la lecture la plus simple auront, plus que les autres, tendance à participer au scrutin.

Lorsqu'une consultation comporte plusieurs enjeux, elle tend ainsi à accroître le poids sur le résultat, du groupe qui a la lecture la plus simple. En d'autres termes, un candidat donné favori par les sondages ou un parti majoritaire dans l'opinion, peut très bien perdre une élection si celle-ci porte sur des enjeux mal perçus par ses partisans. On peut aussi en déduire qu'il aurait pu l'emporter s'il avait fait un effort pédagogique plus poussé en direction de ses électeurs...

\footnotetext{
${ }^{27}$ Le terme de «population homogène » est-il approprié ? L'homogénéité est la propriété d'un ensemble formé d'éléments différents mais de même nature. Appliqué à une population, ce terme a le sens que lui donnait Thiers quand il écrivait «un État aussi homogène et aussi bien fondu que la France, ne pouvait admettre le système fédéral ». Il renvoie à une propriété mathématique utilisée dans l'étude théorique : une transformation linéaire appliquée à un ensemble homogène, ne modifie pas la position relative des éléments qui le composent.

${ }^{28}$ Ces deux configurations comportent par ailleurs un éventail de situations intermédiaires, lorsque les caractéristiques individuelles d'un électeur ne font que conditionner, avec une probabilité plus ou moins grande, son appartenance à l'un des groupes et sa lecture des enjeux.
} 


\section{L’ÉCHEC ÉLECTORAL EST UNE SCIENCE EXACTE}

Dans un contexte de bipolarisation du paysage politique, un candidat n'est jamais tout à fait sûr de gagner une élection, mais il existe un certain nombre de configurations, repérables à l'avance, dans lesquelles il peut être sûr de perdre.

La première d'entre elles, la plus fréquente, consiste à diviser son camp - ou ses variantes: être le candidat d'un camp divisé, ou avoir été désigné à l'issue d'un processus qui a profondément divisé son camp. Surtout s'il est opposé à un candidat qui, lui, n'est pas ou n'est plus dans cette situation.

La cause de l'échec du candidat concerné (que nous appellerons B) tient ici à la superposition de deux phénomènes : la démobilisation, voire l'abstention d'une partie de son électorat naturel - et le comportement des indécis qui, déconcertés par les débats qu'ils ont pu observer et les divisions que ceux-ci révèlent, basculent de l'autre côté.

La division d'un camp n'est cependant pas toujours une condition suffisante. Cette position de faiblesse peut alors être renforcée par un choix stratégique: se présenter « contre $\mathrm{A} »$. Ce positionnement a un avantage : $\mathrm{B}$ fait le plein des opposants à A. Il a un inconvénient : dans une situation classique de bipolarisation, les opposants à A ne forment pas une majorité. Les électeurs qui sont indécis, par définition ne sont pas des inconditionnels de A, mais ils lui accordent leur préférence sur certains enjeux. Il suffit alors pour ce dernier de faire porter sa campagne sur les enjeux en question, pour l'emporter.

L'échec de Ségolène Royal à l'élection présidentielle de 2007 constitue une bonne illustration ce qui vient d'être dit : en s'imposant lors des primaires socialistes comme "le meilleur candidat contre Sarkozy», elle laissait à son adversaire le choix des enjeux sur lesquels elle aurait à l'affronter, au détriment de son propre projet. Elle a fait le plein des opposants à $N$. Sarkozy, mais sans plus. La stratégie choisie pour être présente au premier tour, a créé les conditions de son échec au second tour.

La deuxième manière de perdre une élection consiste à en rendre les enjeux peu compréhensibles. Or, comme nous l'avons mis en évidence au cours de cet exposé, face à des questions complexes la simplicité devient un critère de choix : plus l'enjeu est simple, plus il mobilise les électeurs - et son corollaire : plus les électeurs simplifient les enjeux, plus ils se mobilisent.

Le référendum de 2005 en constitue un exemple particulièrement représentatif. Le débat a porté moins sur la question posée - pour ou contre le projet de Constitution européenne - que sur sa signification implicite : pour ou contre la perte de souveraineté nationale, pour ou contre l'élargissement de l'Europe, pour ou contre son orientation libérale, pour ou contre l'entrée de la Turquie, pour ou contre le gouvernement... Il est clair que si chacune de ces formulations, prise isolément, est lisible, leur superposition ne l'est pas. Les électeurs ont donc eu moins à répondre à une question qu'à choisir le sens à lui donner. Dans ce cas, il est plus facile de répondre non, que oui.

Il existe une troisième manière de perdre une élection - une sorte de variante de la précédente : en minimiser les enjeux. On augmente ainsi l'abstention au sein de ses partisans, sans réduire celle du camp opposé auquel se joindront les indécis, encouragés à manifester un mécontentement à peu de frais - surtout si cette stratégie est celle du parti au pouvoir. 
Cette règle a été parfaitement illustrée en France par les élections régionales de 2004. Ces élections comportaient, comme on l'a déjà rappelé, un double enjeu national et régional : la stratégie adoptée par la majorité au pouvoir a consisté à les présenter comme " des élections locales, dont les résultats n'affecteraient pas les orientations de la politique menée au niveau national ». Les résultats du premier tour ont montré que, pour la majorité des électeurs et des observateurs politiques, il s'agissait bien d'un vote à dimension nationale. La lecture ainsi donnée ne pouvait qu'entraîner au second tour une amplification du mouvement, avec d'un côté une plus forte mobilisation de l'opposition de gauche autour d'un enjeu clair, et de l'autre une plus faible mobilisation des électeurs de la droite, autour d'un enjeu qui restait ambigu. La victoire de la gauche était prévisible, mais elle ne l'était pas dans de telles proportions.

La quatrième façon de perdre une élection, consiste pour le candidat d'un camp à faire campagne au centre (par opposition à la voie royale inverse : être du centre et devenir le candidat d'un camp qui l'acceptera d'autant mieux que, n'appartenant à aucune de ses tendances, il devient le symbole de son unité). Ceci demande à être explicité. Nous avons vu qu'une élection comporte généralement plusieurs enjeux. Chacun des candidats est en mesure de l'emporter si les électeurs privilégient l'enjeu sur lequel son positionnement est le plus favorable. Par exemple: la lutte contre l'insécurité pour un candidat de droite; la protection sociale pour un candidat de gauche. L'issue du scrutin dépendra de la pondération que les électeurs auront faite de ces enjeux. Si le candidat de gauche, pensant élargir son électorat potentiel, s'affirme lui aussi préoccupé par la montée de la délinquance, sa prise de position a pour effet de renforcer le poids de cet enjeu pour les électeurs, et donc indirectement de renforcer la position de son concurrent perçu en tout état de cause comme mieux placé pour répondre à l'inquiétude ainsi soulevée. Il n'est pas impossible, qu'au cours de ce processus, le candidat de gauche gagne quelques voix parmi les indécis, mais il aura rendu indécis une partie de ceux qui, au départ, étaient prêts à voter pour lui.

Pour reprendre la terminologie utilisée dans cet article, une telle stratégie aboutit, à la fois, à diviser l'électorat traditionnel du candidat concerné, et à rendre moins lisible l'enjeu du scrutin. Si, comme cela est souvent le cas, les deux candidats adoptent cette stratégie, le mieux placé pour gagner l'élection est alors celui qui peut le plus facilement ajuster son positionnement à l'attente de ceux qui n'étaient pas spontanément prêts à voter pour lui, sans que le doute ou l'indécision que cela peut entraîner dans son électorat initial lui fassent perdre d'un côté ce qu'il gagne de l'autre.

Ainsi formulé, ce constat paraît signifier qu'un tel positionnement existe pour chacun des candidats. C'est sans doute ce dont chacun d'eux est convaincu, et que leurs conseillers sont chargés de mettre en œuvre. L'observation montre que ce n'est généralement pas le cas, pour au moins deux raisons :

La première raison est qu'un candidat maîtrise relativement peu la pondération des enjeux par les électeurs, et moins encore la perception que ceux-ci peuvent avoir de son positionnement: il est d'autant plus crédible et il mobilise d'autant plus ses partisans que la lecture qu'il donne des enjeux d'un scrutin et de son propre positionnement correspond à leur attente. En modifiant son discours, il prend le risque de ne pas être compris, c'est à dire de ne pas être suivi. Ce qu'on peut exprimer autrement en remarquant que l'électorat qui peut le plus facilement accepter des variations de positionnement de son candidat, est celui qui est le moins sensible aux enjeux spécifiques d'un scrutin, c'est-à-dire l'électorat le moins le moins politisé. 
La seconde raison tient à la manière dont s'organise la bipolarisation d'un paysage politique. Celle-ci prend généralement la forme de vastes coalitions de dirigeants et d'élus se réclamant de sensibilités différentes, organisées ou non en partis, mais affichant un positionnement commun sur les sujets censés déterminer les choix électoraux.

Si l'évolution du contexte politique est telle, que le vote d'une partie des électeurs tend à s'effectuer en fonction d'autres enjeux que ceux autour desquels se sont organisées ces coalitions, ce qui les oppose peut devenir moins déterminant que ce qui distingue entre elles leurs différentes composantes. Il en est de même si le candidat d'un des deux blocs, pour convaincre les indécis, se rapproche des positions de son adversaire. De tels glissements n'entraînent pas seulement, de part et d'autre, l'abstention d'une partie des électeurs, ils débouchent, s'ils prennent de l'ampleur, sur la rupture de la coalition dont ce candidat est censé être le représentant. La coalition la plus hétérogène est naturellement la plus fragile. " Faire le plein des voix de son camp » signifie ici : définir un positionnement qui assure la cohésion d'une coalition, plutôt que l'engager dans des débats qui la divisent.

La dernière élection présidentielle américaine correspond à une configuration de ce type : à l'issue des primaires démocrates Barack Obama, désigné par un parti qui s'était divisé autant sur le choix de son candidat que sur son programme, était virtuellement perdant face à John Mac Cain, représentant peu contesté du parti républicain. Jusqu'au moment où la crise financière a renversé le rapport des forces, en rétablissant l'unité du camp démocrate, face à un électorat républicain désormais divisé sur les réponses à donner à cette crise. On remarquera d'ailleurs que, pour spectaculaire qu'ait pu être la victoire de Barack Obama, il ne l'a emporté sur son adversaire que de quelques pourcents des suffrages exprimés. 


\section{ANNEXE}

\section{NOTATIONS ET DÉFINITIONS}

Cette étude repose sur les propriétés des distributions normales.

Dans tout ce qui suit, u représentant une variable normale réduite (écart type égal à 1), П(U) désigne la fonction cumulative d'une distribution normale, c'est-à-dire la probabilité que u soit inférieure à $\mathrm{U} . \Pi^{\prime}(\mathrm{U})$ représente la dérivée de cette fonction. Une variation $\Delta(\mathrm{U})$ de $\mathrm{u}$, au voisinage de $\mathrm{U}$ entraîne une variation $\Delta[\Pi(\mathrm{U})]$ de $\Pi(\mathrm{U}): \Delta[\Pi(\mathrm{U})]=\Pi^{\prime}\left(\mathrm{U}_{-}\right) . \Delta(\mathrm{U})$.

La déclinaison mathématique des hypothèses introduites met en œuvre une formalisation mathématique relativement complexe et souvent fastidieuse. Nous avons donc préféré utiliser pour cet exposé, chaque fois que cela était possible, la facilité que permet l'approximation de la loi normale П(u) pour une valeur $\mathrm{u}$, fournie par un développement limité à proximité d'une valeur $\mathrm{v}$, et dont l'expression générale est la suivante :

$$
\Pi(u) \cong \Pi(v)+(u-v) . \Pi^{\prime}(v)+1 / 2(u-v)^{2} . \Pi^{\prime},(v)+1 / 6(u-v)^{3} . \Pi^{\prime \prime \prime}(v)+\varepsilon
$$

où $\varepsilon$ est un terme résiduel, d'autant plus faible que $\mathrm{v}$ est proche de $\mathrm{u}$.

Par définition $\Pi^{\prime}(v)=\frac{1}{\sqrt{2 \Pi}} \exp -\frac{1}{2} v^{2}$

d'où :

$$
\begin{gathered}
\Pi^{\prime \prime}(\mathrm{v})=-\mathrm{v} \cdot \Pi^{\prime}(\mathrm{v}) \text { et } \Pi^{\prime \prime \prime}(\mathrm{v})=-\Pi^{\prime}(\mathrm{v})+\mathrm{v}^{2} \cdot \Pi^{\prime}(\mathrm{v}) \\
\Pi(\mathrm{u})=\Pi(\mathrm{v})+(\mathrm{u}-\mathrm{v}) . \Pi^{\prime}(\mathrm{v})-1 / 2(\mathrm{u}-\mathrm{v})^{2} \cdot \mathrm{v} \cdot \Pi^{\prime}(\mathrm{v})+1 / 6(\mathrm{u}-\mathrm{v})^{3} \cdot\left(\mathrm{v}^{2-} 1\right) \cdot \Pi^{\prime}(\mathrm{v})+\varepsilon
\end{gathered}
$$

Dans la quasi-totalité des situations étudiées à partir de ce modèle, les valeurs de u et de v sont proches et relativement faibles. Un, deux, au maximum trois termes du développement limité sont alors suffisants pour obtenir une précision largement supérieure à ce qui est nécessaire :

$$
\Pi(u) \cong \Pi(v)+(u-v) . \Pi^{\prime}(v)-1 / 2(u-v)^{2} \cdot v \cdot \Pi^{\prime}(v)
$$

Les conditions de validité de l'approximation obtenue ne seront précisées que lorsque cela apparaîtra utile, sachant que la validation de la représentation obtenue repose in fine sur la cohérence entre les propriétés théoriques annoncées par le modèle, et les résultats de l'observation.

On en déduit notamment :

$$
\begin{aligned}
& \Pi(\mathrm{u}) \cong \Pi\left(\frac{\mathrm{u}+\mathrm{v}}{2}\right)+\left(\frac{\mathrm{u}-\mathrm{v}}{2}\right) \cdot \Pi^{\prime}\left(\frac{\mathrm{u}+\mathrm{v}}{2}\right)-1 / 2\left(\frac{\mathrm{u}-\mathrm{v}}{2}\right)^{2} \cdot\left(\frac{\mathrm{u}+\mathrm{v}}{2}\right) \cdot \Pi^{\prime}\left(\frac{\mathrm{u}+\mathrm{v}}{2}\right) \\
& \Pi(\mathrm{v}) \cong \Pi\left(\frac{\mathrm{u}+\mathrm{v}}{2}\right)-\left(\frac{\mathrm{u}-\mathrm{v}}{2}\right) \cdot \Pi^{\prime}\left(\frac{\mathrm{u}+\mathrm{v}}{2}\right)-1 / 2\left(\frac{\mathrm{u}-\mathrm{v}}{2}\right)^{2} \cdot\left(\frac{\mathrm{u}+\mathrm{v}}{2}\right) \cdot \Pi^{\prime}\left(\frac{\mathrm{u}+\mathrm{v}}{2}\right)
\end{aligned}
$$

d'où les formules 1 et 2 que nous utiliserons fréquemment :

$$
\begin{gathered}
\Pi(u)-\Pi(v) \cong(u-v) . \Pi^{\prime}\left(\frac{u+v}{2}\right) \\
\Pi(u)+\Pi(v) \cong 2 \Pi\left(\frac{u+v}{2}\right)-\left(\frac{u-v}{2}\right)^{2} \cdot\left(\frac{u+v}{2}\right) \Pi^{\prime}\left(\frac{u+v}{2}\right)
\end{gathered}
$$




\section{PROXIMITÉS RELATIVES ET PROXIMITÉS MOYENNES}

Si nous notons $d_{A}$ et $d_{B}$ les distances respectives d'un électeur quelconque à $\mathrm{A}$ et à $\mathrm{B}$, les proximités relatives, $p_{A / B}$ et $p_{A / B}$, sont par définition données par:

$$
p_{A / B}=d_{B} \cdot d_{A} \quad p_{B / A}=d_{A} \cdot \mathrm{d}_{\mathrm{B}} \quad\left(\text { soit }: p_{A / B}=-p_{B / A}\right)
$$

Un électeur vote A si : $p_{A / B}>s_{A}$

Il vote $B$ si : $\quad p_{B / A}>s_{B}$

$s_{A}$ et $s_{B}$ sont les seuils à partir desquels il est en mesure de décider qu'il est plus proche de l'un que de l'autre. Ces seuils sont supposés statiquement indépendants des proximités relatives.

La mesure de la zone d'indécision, définie par : - $s_{B}<p_{A / B}<s_{A}$ est égale à $\left(s_{A}+s_{B}\right)$

$p_{A / B}$ et $p_{B / A} ; s_{A}$ et $s_{B}$ sont supposées être des variables normales, dont nous noterons les valeurs moyennes $\overline{p_{A / B}}, \overline{p_{B / A}}, \overline{s_{A}}, \overline{s_{B}}$

Les proportions d'électeurs votant soit A soit B sont ainsi données par :

$$
\mathrm{N}(\mathrm{A})=\Pi\left(\frac{\overline{P_{A / B}}-\overline{s_{A}}}{\sqrt{2}}\right) \quad \mathrm{N}(\mathrm{B})=\Pi\left(\frac{\overline{P_{A / B}}-\overline{s_{B}}}{\sqrt{2}}\right)
$$

où $\Pi$ est la fonction cumulative de la loi normale.

Les proximités moyennes $\mathrm{P}_{\mathrm{A} / \mathrm{B}}$ et $\mathrm{P}_{\mathrm{B} / \mathrm{A}}$, telles que nous les avons définies, sont estimées par :

$$
\mathrm{N}(\mathrm{A})=\Pi\left(\mathrm{P}_{\mathrm{A} / \mathrm{B}}\right) \quad \mathrm{N}(\mathrm{B})=\Pi\left(\mathrm{P}_{\mathrm{B} / \mathrm{A}}\right)
$$

Soit :

$$
\mathrm{P}_{\mathrm{A} / \mathrm{B}}+\mathrm{P}_{\mathrm{B} / \mathrm{A}}=-\left(\overline{\frac{s_{A}+s_{B}}{\sqrt{2}}}\right)
$$

On en déduit les propriétés suivantes pour un électorat homogène :

1. L'indice de lisibilité, défini par : $\mathrm{I}=-\left(\mathrm{P}_{\mathrm{A} / \mathrm{B}}+\mathrm{P}_{\mathrm{B} / \mathrm{A}}\right)$, est constant et indépendant des scores des candidats $^{29}$

2. Il existe une relation simple entre le taux d'abstention et l'écart des scores des deux candidats, exprimés en proportion des électeurs inscrit.

Les relations 1 et 2 de la page précédente conduisent à :

$$
\begin{gathered}
\Pi\left(\mathrm{P}_{\mathrm{A} / \mathrm{B}}\right)-\Pi\left(\mathrm{P}_{\mathrm{B} / \mathrm{A}}\right) \cong\left(\mathrm{P}_{\mathrm{A} / \mathrm{B}}-\mathrm{P}_{\mathrm{B} / \mathrm{A}}\right) \cdot \Pi^{\prime}\left(\frac{\mathrm{P}_{\mathrm{A} / \mathrm{B}}+\mathrm{P}_{\mathrm{B} / \mathrm{A}}}{2}\right) \\
\Pi\left(\mathrm{P}_{\mathrm{A} / \mathrm{B}}\right)+\Pi\left(\mathrm{P}_{\mathrm{A} / \mathrm{B}}\right) \cong 2 \Pi\left(\frac{\mathrm{P}_{\mathrm{A} / \mathrm{B}}+\mathrm{P}_{\mathrm{B} / \mathrm{A}}}{2}\right)-\left(\frac{\mathrm{P}_{\mathrm{A} / \mathrm{B}}-\mathrm{P}_{\mathrm{B} / \mathrm{A}}}{2}\right)^{2} \cdot\left(\frac{\mathrm{P}_{\mathrm{A} / \mathrm{B}}+\mathrm{P}_{\mathrm{B} / \mathrm{A}}}{2}\right) \cdot \Pi^{\prime}\left(\frac{\mathrm{P}_{\mathrm{A} / \mathrm{B}}+\mathrm{P}_{\mathrm{B} / \mathrm{A}}}{2}\right)
\end{gathered}
$$

Soit :

$$
\begin{gathered}
N(A)-N(B) \cong\left(P_{A / B}-P_{B / A}\right) \cdot \Pi^{\prime}(I / 2) \\
N_{=}=1-[N(A)+N(B)] \cong 1-2 \Pi[-I / 2]-\left(\frac{P_{A / B}-P_{B} / A}{2}\right)^{2} \cdot I / 2 . \Pi^{\prime}(I / 2)
\end{gathered}
$$

Posant : $S=N(A)-N(B)$

$$
\mathrm{N}_{=} \cong \alpha-\beta \cdot \mathrm{S}^{2}
$$

${ }^{29} \mathrm{P}_{\mathrm{A} / \mathrm{B}}+\mathrm{P}_{\mathrm{B} / \mathrm{A}}$ est nécessairement négatif. Par convention l'Indice de lisibilité est égal à sa valeur absolue. 
où :

$$
\alpha=1-2 \Pi[-1 / 2 \mathrm{I}] \quad \beta=\mathrm{I} / 8 . \Pi^{\prime}(\mathrm{I} / 2)
$$

$\alpha$, maximum de $\mathrm{N}_{=}$, correspond à $\mathrm{S}=0$.

On a alors :

$$
\mathrm{N}(\mathrm{A})=\mathrm{N}(\mathrm{B})=\Pi[-1 / 2 \mathrm{I}]
$$

3. Dans la plupart des configurations étudiées, l'indice de lisibilité est inférieur à 0,9 , justifiant les approximations suivantes ${ }^{30}$ :

$$
\begin{gathered}
\alpha \cong 0,4 \text {. I } \quad \beta \cong 0,8 \cdot \alpha \\
\left.\mathrm{N}_{=} \cong 0,4 \text {. I. [ } 1-0,8 \cdot \mathrm{S}^{2}\right]
\end{gathered}
$$

Lorsque le solde des réponses est relativement faible, $\mathrm{N}_{=}$est pratiquement proportionnel à l'indice de lisibilité. Il diminue quand l'écart se creuse entre $\mathrm{N}(\mathrm{A})$ et $\mathrm{N}(\mathrm{B})$, comme le carré de cet écart.

- Les tests de l'homogénéité de l'électorat

Les relations précédentes fournissent des tests de validité des hypothèses introduites, et notamment de l'homogénéité de l'électorat pour le scrutin concerné ${ }^{31}$.

Le premier de ces tests consiste à vérifier que la somme des proximités moyennes peut être considérée comme constante, d'une circonscription à l'autre.

Le deuxième test porte sur la relation entre $\mathrm{N}_{=}$et le solde des réponses $\mathrm{S}$. Le maximum de $N_{=}$doit en principe être observé quand $N(A)=N(B)$, et il doit diminuer comme le carré du solde des réponses. Ce test ne peut toutefois être significatif que si l'écart entre N(A) et N(B), est suffisamment important pour que l'incidence des aléas statistiques devienne négligeable.

\section{ABSTENTION THÉORIQUE, ABSTENTION STRUCTURELLE, ABSTENTION OBSERVÉE}

La détermination des différents paramètres de la représentation utilisée, est généralement biaisée par l'abstention structurelle.

Par rapport aux électeurs inscrits, la représentation utilisée ne prend en effet en compte que les électeurs qui votent ou qui s'abstiennent en fonction des options qui leur sont proposées, soit une proportion $\mu$ des inscrits, où $\mu=\left(1-\mathrm{N}_{=s}\right)$ et où $\mathrm{N}_{=\mathrm{s}}$ représente le taux d'abstention structurelle.

$\mathrm{N}(\mathrm{A}), \mathrm{N}(\mathrm{B}), \mathrm{N}_{-}, \mathrm{S}$ - scores, taux d'abstention et écarts observés - sont alors liés aux valeurs théoriques $\mathrm{N}^{*}(\mathrm{~A}), \mathrm{N}^{*}(\mathrm{~B}), \mathrm{N}^{*}, \mathrm{~S}^{*}$ par les relations suivantes :

30

\begin{tabular}{|l|l|l|l|l|l|l|l|}
\hline $\mathrm{I}$ & 0,3 & 0,4 & 0,5 & 0,6 & 0,7 & 0,8 & 0,9 \\
\hline$\Pi^{\prime}(\mathrm{I} / 2)$ & 0,395 & 0,391 & 0,387 & 0,381 & 0,375 & 0,368 & 0,361 \\
\hline$\Pi^{\prime}(0)$ & 0,399 & 0,399 & 0,399 & 0,399 & 0,399 & 0,399 & 0,399 \\
\hline écart & $1 \%$ & $2 \%$ & $3 \%$ & $5 \%$ & $6 \%$ & $8 \%$ & $11 \%$ \\
\hline$\alpha$ & 0,119 & 0,159 & 0,197 & 0,236 & 0,274 & 0,312 & 0,347 \\
\hline $0,4 . \mathrm{I}$ & 0,12 & 0,16 & 0,2 & 0,24 & 0,28 & 0,32 & 0,36 \\
\hline Ecart & $0,6 \%$ & $0,9 \%$ & $1,3 \%$ & $1,8 \%$ & $2,3 \%$ & $2,9 \%$ & $3,6 \%$ \\
\hline$\beta$ & 0,0949 & 0,128 & 0,162 & 0,197 & 0,233 & 0,271 & 0,311 \\
\hline $0,8 \alpha$ & 0,0952 & 0,127 & 0,158 & 0,189 & 0,219 & 0,250 & 0,278 \\
\hline écart & $0,03 \%$ & $0,05 \%$ & $2,5 \%$ & $4 \%$ & $6 \%$ & $9 \%$ & $12 \%$ \\
\hline
\end{tabular}

${ }^{31}$ En fait, comme souvent dans les analyses statistiques, les tests de vraisemblance permettent moins de prouver la validité du modèle théorique, que de préciser les conditions dans lesquelles il est possible de ne pas l'invalider. C'est le degré de concordance entre les applications que l'on peut en tirer et les résultats de l'observation qui détermine, in fine, son degré d'adéquation à la réalité étudiée. 


$$
\begin{gathered}
\mathrm{N}(\mathrm{A})=\mu \mathrm{N}^{*}(\mathrm{~A}) \quad \mathrm{N}(\mathrm{B})=\mu \mathrm{N}^{*}(\mathrm{~B}) \\
\left(1-\mathrm{N}_{=}\right)=\mu\left(1-\mathrm{N}^{*}\right) \quad \mathrm{S}=\mu \mathrm{S}^{*}
\end{gathered}
$$

On remarquera que le score relatif, $S$ / $\left(1-N_{=}\right)$est indépendant de l'abstention structurelle.

\section{Incidence de l'abstention structurelle sur l'évaluation de l'indice de lisibilitée}

Les proximités moyennes, a et $b$, d'une part, $a^{*}$ et $b^{*}$ d'autre part sont par définition données par :

$$
\Pi(a)=N(A) \quad \Pi(b)=N(B) \quad \Pi\left(a^{*}\right)=N^{*}(A) \quad \Pi\left(b^{*}\right)=N^{*}(A)
$$

On en déduit :

$$
\Pi(\mathrm{a})=\mu . \Pi\left(\mathrm{a}^{*}\right) \quad \text { et } \quad \Pi(\mathrm{b})=\mu \cdot \Pi\left(\mathrm{b}^{*}\right)
$$

Un développement limité conduit à :

$$
\Pi(\mathrm{a})=\Pi\left(\mathrm{a}^{*}+\mathrm{a}-\mathrm{a}^{*}\right)=\Pi\left(\mathrm{a}^{*}\right)+\left(\mathrm{a}-\mathrm{a}^{*}\right) \cdot \Pi^{\prime}\left(\mathrm{a}^{*}\right)=\mu \Pi\left(\mathrm{a}^{*}\right)
$$

soit :

$$
\left(\mathrm{a}-\mathrm{a}^{*}\right) . \Pi^{\prime}\left(\mathrm{a}^{*}\right)=-(1-\mu) . \Pi\left(\mathrm{a}^{*}\right)
$$

$$
\mathrm{a}=\mathrm{a}^{*}-(1-\mu) . \Pi\left(\mathrm{a}^{*}\right) / \Pi^{\prime}\left(\mathrm{a}^{*}\right) \quad \mathrm{b}=\mathrm{b}^{*}-(1-\mu) . \Pi\left(\mathrm{b}^{*}\right) / \Pi^{\prime}\left(\mathrm{b}^{*}\right)
$$

Les indices de lisibilité sont donnés par :

$$
\begin{gathered}
\mathrm{I}=-(\mathrm{a}+\mathrm{b}) \quad \text { et } \mathrm{I}^{*}=-\left(\mathrm{a}^{*}+\mathrm{b}^{*}\right) \\
\mathrm{I}=\mathrm{I}^{*}+(1-\mu) \cdot\left[\Pi\left(\mathrm{a}^{*}\right) / \Pi^{\prime}\left(\mathrm{a}^{*}\right)+\Pi\left(\mathrm{b}^{*}\right) / \Pi^{\prime}\left(\mathrm{b}^{*}\right)\right] \\
\mathrm{I} \cong \mathrm{I}^{*}+2 \mathrm{~N}_{\mathrm{s}} \cdot \Pi\left(-\mathrm{I}^{*} / 2\right) / \Pi^{\prime}\left(\mathrm{I}^{*} / 2\right)
\end{gathered}
$$

L'abstention structurelle a pour effet d'introduire un biais dans l'évaluation de l'indice de lisibilité : la valeur estimée est supérieure à celle qui correspond à l'électorat effectif. La lisibilité du scrutin semble plus faible qu'elle n'est en réalité.

Les fluctuations de l'abstention culturelle se superposent à celles de l'indice de lisibilité. L’hétérogénéité apparente de l'électorat est plus importante que son hétérogénéité réelle.

2. Incidence de l'abstention structurelle sur l'évaluation de la relation entre le score des candidats et l'abstention

Compte tenu des relations entre les scores des candidats et les taux d'abstention, théoriques et observés, l'expression théorique $\left(1-\mathrm{N}^{*}\right) \cong 1-\alpha^{*}+\beta^{*}$. $\mathrm{S}^{* 2}$ conduit, pour une valeur donnée de $\mu$, à :

$$
\left(1-\mathrm{N}_{=}\right) / \mu=\left(1-\alpha^{*}\right)+\left(\beta^{*} / \mu^{2}\right) \mathrm{S}^{2} \text { d'où }\left(1-\mathrm{N}_{=}\right)=\mu\left(1-\alpha^{*}\right)+(\beta * / \mu) . \mathrm{S}^{2}
$$

soit :

$$
\mathrm{N}_{=} \cong \alpha-\beta \cdot \mathrm{S}^{2}
$$

avec : $\quad \beta=\beta^{*} / \mu$ et $1-\alpha=\mu\left(1-\alpha^{*}\right)$

Le rapprochement de ces deux relations conduit à :

$$
\left(1-\alpha^{*}\right) \beta^{*}=(1-\alpha) \beta
$$

On déduit de ce qui précède plusieurs conséquences.

2.1. Si l'électorat est homogène et si l'observation porte sur des agrégats obtenus en regroupant les circonscriptions en fonction d'un critère indépendant de $\mu$, en nombre suffisant pour que sa variabilité soit faible devant celle de $\mathrm{S}$ et $\mathrm{N}_{=}$, $\alpha$ et $\beta$ sont en principe (statistiquement) constants : il doit exister entre les valeurs observées $N_{=}$et $S$, une relation semblable à celle qui existe entre les valeurs théoriques. En particulier, la valeur moyenne de $\mathrm{N}_{=}$tend à diminuer quand la valeur moyenne de $\mathrm{S}$ augmente.

\footnotetext{
${ }^{32}$ On suppose dans tout ce qui suit que l'électorat est homogène, c'est-à-dire que l'écart entre la représentation théorique et la représentation réelle tient essentiellement au biais introduit par l'abstention structurelle.
} 
Inversement, lorsque cette proposition est vérifiée, $\mu$ peut être considéré comme statistiquement indépendant du critère de regroupement utilisé.

2.2. On peut alors évaluer l'indice de lisibilité théorique I* à partir de la relation :

$$
(1-\alpha) \beta=\left(1-\alpha^{*}\right) \beta^{*}=1 / 2 \Pi\left[I^{*} / 2\right] .\left(I^{*} / 2\right) / \Pi^{\prime}\left(I^{*} / 2\right)
$$

en utilisant le tableau de correspondance suivant, établi à partir des tables numériques de la loi normale :

\begin{tabular}{|c|c|c|c|c|c|c|c|}
\hline$(1-\alpha) \beta$ & $-0,084$ & $-0,108$ & $-0,13$ & $-0,15$ & $-0,169$ & $-0,187$ & $-0,204$ \\
\hline$I^{*}$ & 0,3 & 0,4 & 0,5 & 0,6 & 0,7 & 0,8 & 0,9 \\
\hline
\end{tabular}

2.3. On peut de même obtenir une estimation du taux d'abstention structurelle.

La valeur estimée de I* permet d'évaluer $\alpha^{*}=0,4$. I*.

On en déduit $1-\mathrm{N}_{=\mathrm{s}}=\mu=(1-\alpha) /\left(1-\alpha^{*}\right)$ soit :

$$
\mathrm{N}_{=\mathrm{s}}=1-(1-\alpha) /\left(1-\alpha^{*}\right)
$$

- L'ensemble de ce qui précède peut être illustré à partir des résultats du second tour de l'Élection présidentielle de 2002

Les deux graphiques ci-après représentent la relation entre le taux d'abstention observé et le carré des écarts des scores des deux candidats (calculés sur les suffrages exprimés).

Le premier des graphiques est établi à partir des résultats observés sur chacun des départements de la France métropolitaine.

Le second est établi à partir des résultats moyens obtenus sur des groupes de 10 départements classés en fonction de l'écart des scores obtenus par les deux candidats, de manière à réduire l'impact de l'abstention structurelle. La tendance annoncée par le modèle théorique y apparaît beaucoup plus nettement, ce qui signifie à la fois, que l'électorat peut être décrit comme plutôt homogène et que, au niveau des départements, l'abstention culturelle peut être considérée comme largement indépendante (au sens statistique) du choix des électeurs.

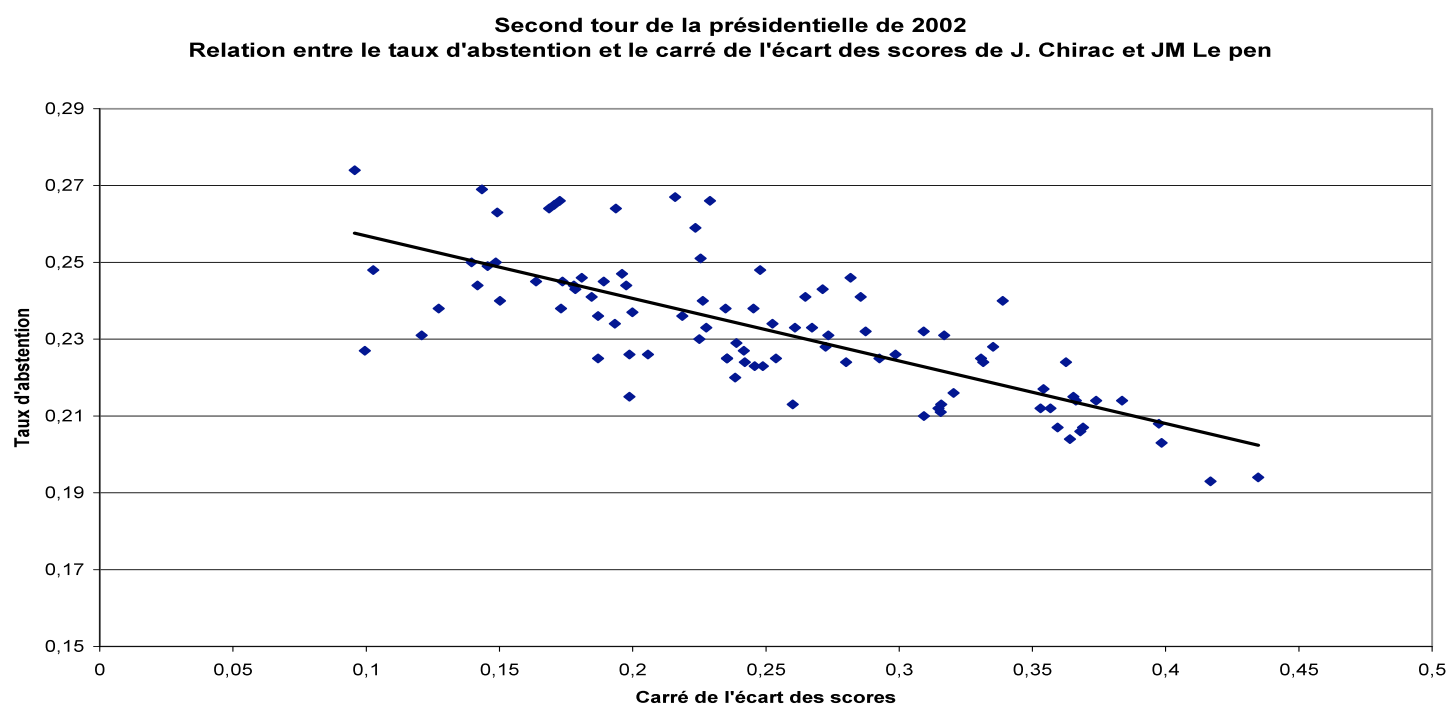




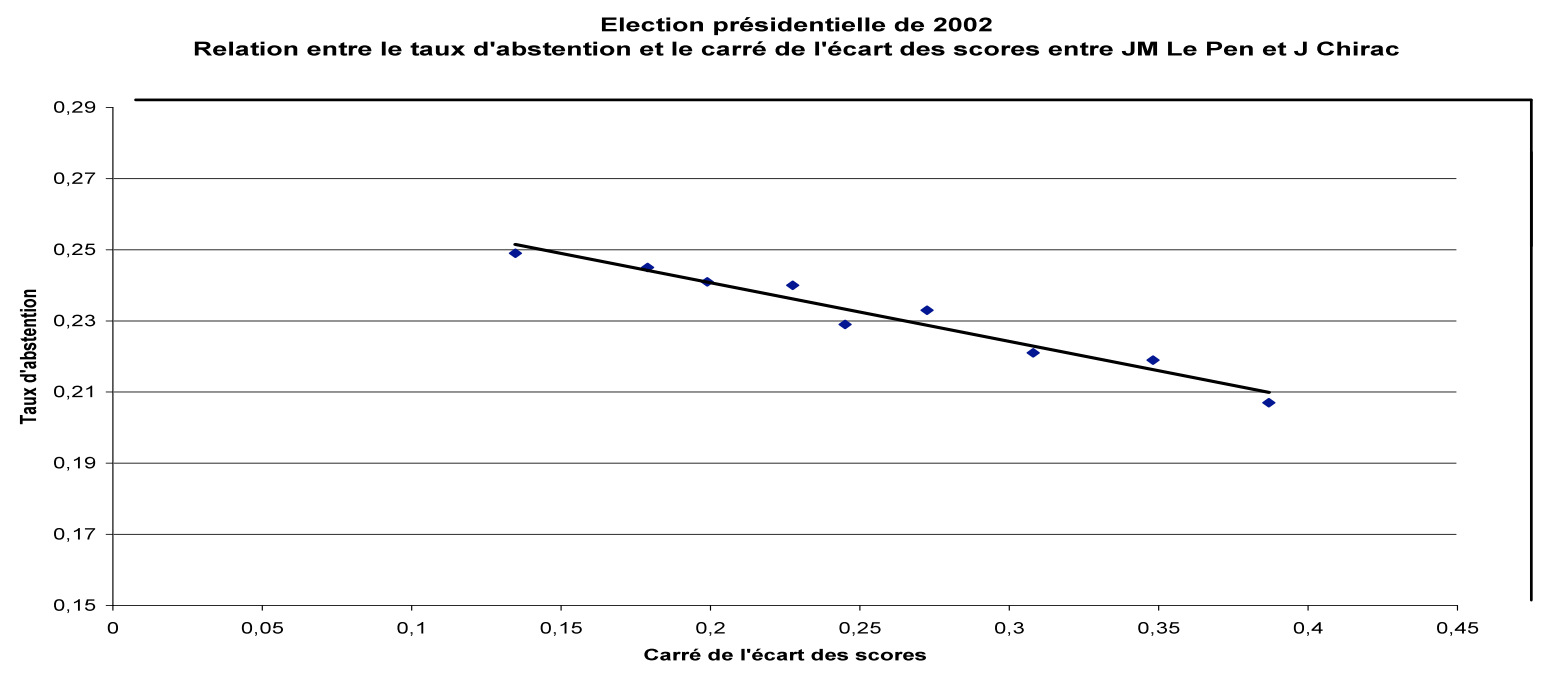

- Estimation de la lisibilité réelle et du taux d'abstention structurelle

\begin{tabular}{|c|c|c|c|c|c|}
\hline \multicolumn{6}{|c|}{ Résultats du second tour de la présidentielle de 2002 , en proportion du nombre des inscrits } \\
\hline \multirow{10}{*}{$\begin{array}{l}\text { Moyennes calculées } \\
\text { sur des groupes de } \\
\text { dix départements } \\
\text { classés en fonction } \\
\text { de la différence de } \\
\text { scores entre les } \\
\text { candidats. }\end{array}$} & Abstentions & Chirac / inscrits & Le Pen/ /inscrits & écart & Carré de l'écart \\
\hline & 0,245 & 0,555 & 0,2 & 0,355 & $\mathbf{0 , 1 2 5 9}$ \\
\hline & 0,259 & 0,573 & 0,168 & 0,405 & 0,1637 \\
\hline & 0,24 & 0,597 & 0,163 & 0,433 & 0,1877 \\
\hline & 0,238 & 0,613 & 0,149 & 0,463 & 0,2146 \\
\hline & 0,253 & 0,61 & 0,137 & 0,474 & 0,2243 \\
\hline & 0,234 & 0,635 & 0,131 & 0,504 & $\mathbf{0 , 2 5 4}$ \\
\hline & 0,232 & 0,65 & 0,118 & 0,533 & 0,2841 \\
\hline & 0,22 & 0,675 & 0,105 & 0,569 & 0,3242 \\
\hline & 0,215 & 0,692 & 0,093 & 0,6 & 0,3597 \\
\hline
\end{tabular}

Il existe une corrélation nette $(-0,85)$ entre la proportion d'abstentions et le carré des écarts de scores entre J. Chirac et J.-M. Le Pen.

Un calcul de régression entre $\mathrm{N}_{=}$et $\mathrm{S}^{2}$ conduit à :

$$
\begin{gathered}
\mathrm{N}_{=}=0,275-0,160 \mathrm{~S}^{2} \quad \text { d'où } \quad \alpha=0,275 \quad \beta=0,174 \\
0,4 . \mathrm{I}=0,275 \quad \text { soit }: \mathrm{I}=0,69
\end{gathered}
$$

La lisibilité apparente correspond à un indice de lisibilité de 0,69

$$
\beta *(1-\alpha *)=\beta(1-\alpha)=0,126 \quad \text { soit } \quad \mathrm{I}^{*}=0,48
$$

La lisibilité réelle correspond à un indice de lisibilité de 0,48

L'abstention observée est de $23,7 \%$

Le taux moyen d'abstention structurelle, $\mathrm{N}_{=\mathrm{s}}$, est donné par $\mathrm{N}_{=\mathrm{s}}=1-(1-\alpha) /\left(1-\alpha^{*}\right)$

$$
\mathrm{N}_{=\mathrm{s}}=10,5 \%
$$

Au second tour de l'élection présidentielle de 2002, l'abstention structurelle a représenté près de la moitié de l'abstention totale, l'autre moitié étant représentée par les électeurs n'ayant pas voulu choisir entre J. Chirac et J.-M. Le Pen. 


\section{ENJEUX MULTIPLES}

Nous supposons que la consultation qui oppose deux candidats A et B, comporte deux enjeux principaux (x) et (y), indépendants, dont les poids respectifs sont notés $\mathrm{p}_{\mathrm{x}}$ et $\mathrm{p}_{\mathrm{y}}{ }^{33}$

L'analyse qui suit repose sur les propriétés générales des lois normales et notamment sur le fait que, si $\mathrm{X}$ et $\mathrm{Y}$ sont deux variables normales réduites indépendantes, $\mathrm{Z}=\mathrm{X} \cos \phi+\mathrm{Y} \sin \phi$ est une variable normale réduite.

Les enjeux de référence $(\mathrm{x})$ et $(\mathrm{y})$ sont représentés par deux axes perpendiculaires, Ox et Oy. Une combinaison $(\mathrm{z})$ de ces enjeux est représentée par l'axe $\mathrm{Oz}$, formant un angle $\theta$ avec $\mathrm{Ox}$ :

$$
\mathrm{p}_{\mathrm{y} / \mathrm{x}} \mathrm{p}_{\mathrm{x}}=\sin \theta / \cos \theta=\operatorname{tg} \theta=\mathrm{t} \text { (t est toujours positif) }
$$

Les proximités relatives correspondantes : $\mathrm{u}_{\mathrm{x}-}$ et $\mathrm{u}_{\mathrm{x}+} ; \mathrm{u}_{\mathrm{y}-}$ et $\mathrm{u}_{\mathrm{y}+} ; \mathrm{u}_{\mathrm{z}-}$ et $\mathrm{u}_{\mathrm{z}+}$ sont des variables normales dont l'écart type est, par définition, égal à 1. Leur relation est donnée par :

$$
\mathrm{u}_{\mathrm{z}-}=\mathrm{u}_{\mathrm{x}-} \cdot \cos \theta+\mathrm{u}_{\mathrm{y}-} \cdot \sin \theta \quad \mathrm{u}_{\mathrm{z}+}=\mathrm{u}_{\mathrm{x}+} \cdot \cos \theta+\mathrm{u}_{\mathrm{y}+} \cdot \sin \theta
$$

On en déduit l'expression de $\mathrm{I}_{\mathrm{z}}$, indice de lisibilité associé à (z)

$$
\begin{array}{lr}
\mathrm{I}_{\mathrm{x}}=\mathrm{u}_{\mathrm{x}-}+\mathrm{u}_{\mathrm{x}+} \quad \mathrm{I}_{\mathrm{y}}=\mathrm{u}_{\mathrm{y}-}+\mathrm{u}_{\mathrm{y}+} \\
\mathrm{I}_{\mathrm{z}}=\mathrm{u}_{\mathrm{z}-}+\mathrm{u}_{\mathrm{z}+}=\mathrm{I}_{\mathrm{x}} \cdot \cos \theta+\mathrm{I}_{\mathrm{y}} \cdot \sin \theta
\end{array}
$$

- Dans les cas où les lisibilités des deux enjeux sont du même ordre : $\mathrm{I}_{\mathrm{x}} \cong \mathrm{I}_{\mathrm{y}}$

$$
\begin{gathered}
I_{z} \cong I_{x} \cdot[\cos \theta+\sin \theta] \\
I_{z}{ }^{2} \cong I_{x}{ }^{2} \cdot[\cos \theta+\sin \theta]^{2}=I_{x}{ }^{2} \cdot[1+2 \cdot \cos \theta \cdot \sin \theta] \\
I_{z} \cong I_{x} \cdot \sqrt{[1+\sin 2 \theta]}=I_{x} \cdot \sqrt{1+2 t /\left(1+t^{2}\right)}
\end{gathered}
$$

L'indice de lisibilité de l'enjeu pondéré est supérieur à l'indice de lisibilité de chacun des enjeux élémentaires.

En d'autres termes, la lisibilité d'un scrutin dans lequel les électeurs doivent prendre en compte deux enjeux indépendants l'un de l'autre et de lisibilité comparable, est inférieure à ce qu'elle serait si les électeurs se déterminaient selon un enjeu unique ${ }^{34}$.

L'augmentation de la propension à l'abstention qui en résulte est proportionnelle à celle de l'indice de lisibilité. Ainsi, si les électeurs donnent le même poids aux deux enjeux, l'abstention augmente d'environ $40 \%{ }^{35}$.

\section{V.ÉLECTORAT NON HOMOGÈNE}

Nous supposons l'électorat formé de deux groupes homogènes distincts, (1) et (2), présents dans les différentes circonscriptions, dans des proportions variables $\mathrm{q}_{1}$ et $\mathrm{q}_{2}$, où $\mathrm{q}_{1}+\mathrm{q}_{2}=1$.

L'élection oppose deux candidats A et B. Dans une circonscription donnée, leurs scores exprimés en proportion des inscrits, sont respectivement $\mathrm{N}(\mathrm{A})$ et $\mathrm{N}(\mathrm{B})$ pour l'ensemble de l'électorat ; $\mathrm{N}_{1}(\mathrm{~A})$ et $\mathrm{N}_{1}(\mathrm{~B})$ dans le groupe (1); $\mathrm{N}_{2}(\mathrm{~A})$ et $\mathrm{N}_{2}(\mathrm{~B})$ dans le groupe (2).

$$
\mathrm{N}(\mathrm{A})=\mathrm{q}_{1} \cdot \mathrm{N}_{1}(\mathrm{~A})+\mathrm{q}_{2} \cdot \mathrm{N}_{2}(\mathrm{~A}) \quad \text { et } \quad \mathrm{N}(\mathrm{B})=\mathrm{q}_{1} \cdot \mathrm{N}_{1}(\mathrm{~B})+\mathrm{q}_{2} \cdot \mathrm{N}_{2}(\mathrm{~B})
$$

Ce qui, exprimé en fonction des proximités moyennes, $a_{1}$ et $b_{1}, a_{2}$ et $b_{2}$, conduit à :

$$
\prod(a)=q_{1} \cdot \prod\left(a_{1}\right)+q_{2} \cdot \prod\left(a_{2}\right) \quad \text { et } \quad \prod(b)=q_{1} \cdot \prod\left(b_{1}\right)+q_{2} \cdot \prod\left(b_{2}\right)
$$

\footnotetext{
${ }^{33}$ On obtient des résultats similaires si les deux enjeux ne sont pas indépendants.

${ }^{34} \mathrm{Si}$ l'un des enjeux a une lisibilité nettement supérieure à l'autre on montre, de la même manière, que la lisibilité de l'enjeu pondéré est toujours inférieure à celle de l'enjeu le plus lisible.

${ }^{35} \mathrm{Si}$ les électeurs donnent le même poids aux deux enjeux $\theta=. \Pi / 4$ soit $: \sin 2 \theta=1 \quad I_{z}=I_{x} \cdot \sqrt{2}=1,4 . I_{x}$.
} 
Nous nous proposons de montrer que a est pratiquement égal à $a^{*}=q_{1} \cdot a_{1}+q_{2} \cdot a_{2}$.

Un développement limité autour de $\mathrm{a}^{*}$ conduit en effet à :

$$
\begin{aligned}
& \prod\left(a_{1}\right) \cong \prod\left(a^{*}\right)+\left(a_{1}-a^{*}\right) \prod '\left(a^{*}\right)-1 / 2\left(a_{1}-a^{*}\right)^{2} a^{*} \prod '\left(a^{*}\right) \\
& \prod\left(a_{2}\right) \cong \prod\left(a^{*}\right)+\left(a_{2}-a^{*}\right) \prod '\left(a^{*}\right)-1 / 2\left(a_{2}-a^{*}\right)^{2} a^{*} \prod^{\prime}\left(a^{*}\right)
\end{aligned}
$$

Or, $\left(a^{*}-a_{1}\right)=\left(q_{1}-1\right) \cdot a_{1}+q_{2} \cdot a_{2}=q_{2} \cdot\left(a_{2}-a_{1}\right)$ et $\left(a^{*}-a_{2}\right)=\left(q_{2}-1\right) \cdot a_{1}+q_{2} \cdot a_{2}=q_{1} \cdot\left(a_{1}-a_{2}\right)$

Ce qui conduit à : $\quad \prod(\mathrm{a}) \cong \prod\left(\mathrm{a}^{*}\right)-\mathrm{q}_{1} \mathrm{q}_{2} \cdot\left(\mathrm{a}_{2}-\mathrm{a}_{1}\right)^{2} \mathrm{a}^{*} \prod{ }^{\prime}\left(\mathrm{a}^{*}\right)$ : d'où

$$
\mathrm{a} \cong \mathrm{a}^{*}-\mathrm{q}_{1} \mathrm{q}_{2} \cdot\left(\mathrm{a}_{2}-\mathrm{a}_{1}\right)^{2} \mathrm{a}^{*}=\mathrm{a}^{*}\left[1-\mathrm{q}_{1} \mathrm{q}_{2} \cdot\left(\mathrm{a}_{2}-\mathrm{a}_{1}\right)^{2}\right]
$$

De même un développement limité autour de $b^{*}=q_{1} \cdot b_{1}+q_{2} \cdot b_{2}$ conduit à :

$$
\mathrm{b}=\mathrm{b}^{*}-\mathrm{q}_{1} \mathrm{q}_{2} \cdot\left(\mathrm{b}_{2}-\mathrm{b}_{1}\right)^{2} \quad \mathrm{~b}^{*}=\mathrm{b}^{*}\left[1-\mathrm{q}_{1} \mathrm{q}_{2} \cdot\left(\mathrm{b}_{2}-\mathrm{b}_{1}\right)^{2}\right]
$$

Compte tenu du degré d'approximation auquel nous nous plaçons, $\mathrm{q}_{1} \mathrm{q}_{2}\left(\mathrm{a}_{2}-\mathrm{a}_{1}\right)^{2}$ et $\mathrm{q}_{1} \mathrm{q}_{2}\left(\mathrm{~b}_{2}-\mathrm{b}_{1}\right)^{2}$ peuvent être considérés comme négligeables par rapports à $1^{36}$ :

$$
a \cong b^{*}=q_{1} \cdot a_{1}+q_{2} \cdot a_{2} \quad b \cong b^{*}=q_{1} \cdot b_{1}+q_{2} \cdot b_{2}
$$

On en déduit l'expression de l'indice de lisibilité de l'ensemble, à partir des indices de chacun des deux groupes : $I_{1}=a_{1}+b_{1}$ et $I_{2}=a_{2}+b_{2}$

$$
\begin{gathered}
I \cong q_{1} \cdot I_{1}+q_{2} \cdot I_{2} \\
I=1 / 2\left(I_{1}+I_{2}+1 / 2\left(I_{1}-I_{2}\right)\left(q_{1}-q_{2}\right)\right.
\end{gathered}
$$

Les deux groupes étant supposés homogènes, $\mathrm{I}_{1}$ et $\mathrm{I}_{2}$ peuvent être considérés comme constants. L'indice de lisibilité de l'ensemble apparaît ainsi comme une fonction linéaire du poids relatif des groupes en présence. Sa valeur diminue quand le poids du groupe ayant l'indice de lisibilité le plus faible, augmente.

En pratique, cette tendance peut être en partie masquée, comme nous l'avons vu, par les fluctuations de l'abstention structurelle. Mais elle doit pouvoir être mise en évidence sur les valeurs moyennes, quand celles-ci concernent des agrégats de taille suffisante.

1. On en déduit qu'il doit exister une corrélation entre la valeur de l'indice de lisibilité et le poids relatif des groupes étudiés, et que cette corrélation doit apparaître encore plus nettement, quand la procédure statistique utilisée réduit l'incidence du biais lié à l'abstention structurelle. On peut alors établir une régression linéaire entre $\mathrm{I}$ et $\mathrm{q}_{1}, \mathrm{q}_{2}$ de la forme :

$$
\mathrm{I} \cong \mu_{1}+\mu_{2} \cdot\left(\mathrm{q}_{1}-\mathrm{q}_{2}\right)
$$

D'où on tire : $\quad I_{1} \cong \mu_{1}+\mu_{2} \quad$ et $\quad I_{2} \cong \mu_{1}-\mu_{2}$

Si (1) est le groupe qui a la meilleure lisibilité, $\mu_{1}+\mu_{2}<\mu_{1}-\mu_{2} \rightarrow \mu_{2}$ est négatif.

2. L'application la plus intéressante de l'analyse qui vient d'être faite concerne les élections dans lesquelles la partition en deux groupes recoupe les électorats des deux candidats.

Dans ce cas, les proportions $\mathrm{q}_{1}$ et $\mathrm{q}_{2}$ peuvent, en première approximation, être assimilées aux proportions $\mathrm{n}(\mathrm{A})$ et $\mathrm{n}(\mathrm{B})$ des suffrages exprimés allant à chacun des candidats ${ }^{37}$ :

$$
\left(\mathrm{q}_{1}-\mathrm{q}_{2}\right) \cong[\mathrm{n}(\mathrm{A})-\mathrm{n}(\mathrm{B})]=[\mathrm{N}(\mathrm{A})-\mathrm{N}(\mathrm{B})] /\left(1-\mathrm{N}_{=}\right)=\mathrm{S} /\left(1-\mathrm{N}_{=}\right)
$$

On doit pouvoir observer une corrélation nette entre l'indice de lisibilité, et la différence des scores relatifs des candidats (une telle corrélation fournit un test de validité de la représentation utilisée, pour un électorat non homogène).

\footnotetext{
${ }^{36}$ Un calcul plus développé, et qu'il est inutile de reprendre ici, montre que cette approximation est effectivement justifiée.

${ }^{37}$ Ce qui correspond à : $\mathrm{q}_{1}+\mathrm{q}_{2}=\mathrm{n}(\mathrm{A})+\mathrm{n}(\mathrm{B})=1$.
} 
3. Quand l'électorat n'est pas homogène, le maximum du taux d'abstention n'est pas observé quand les scores des deux candidats sont identiques.

$$
I=1 / 2\left(I_{1}+I_{2)}+1 / 2\left(I_{1}-I_{2}\right)\left(q_{1}-q_{2}\right) \quad S=\left(1-N_{=}\right) \cdot\left(q_{1}-q_{2}\right)\right.
$$

D'après la relation générale $\quad \mathrm{N}_{=} \cong \alpha-\beta . \mathrm{S}^{2} \cong 0,4 \cdot \mathrm{I}\left[1-0,8 . \mathrm{S}^{2}\right]$

Si l'électorat est suffisamment hétérogène, c'est-à-dire si les indices de lisibilité $\mathrm{I}_{1}$ et $\mathrm{I}_{2}$ sont suffisamment différents, les variations de I sont nettement plus marquées que celles de $\mathrm{S}^{2}$.

On doit pouvoir vérifier que le taux d'abstention est alors pratiquement proportionnel à I.

Le maximum de taux d'abstention doit s'observer quand l'indice de lisibilité est à son maximum, c'està-dire quand le candidat qui a le plus de voix est celui dont l'électorat présente l'indice de lisibilité le plus élevé (donc la lisibilité des enjeux la plus faible).

L'ensemble de ce qui précède peut être illustré à partir des résultats du second tour de l'élection présidentielle de 2007

Le graphique ci-dessous représente le taux d'abstention en fonction du carré de l'écart des scores de S. Royal et N. Sarkozy dans les différents départements de la France métropolitaine. Aucune tendance n'apparaît : soit l'électorat n'est pas homogène, soit l'écart de score des deux candidats étant, le plus souvent, relativement peu important, la tendance annoncée par le modèle théorique est masquée par les fluctuations de l'abstention structurelle.

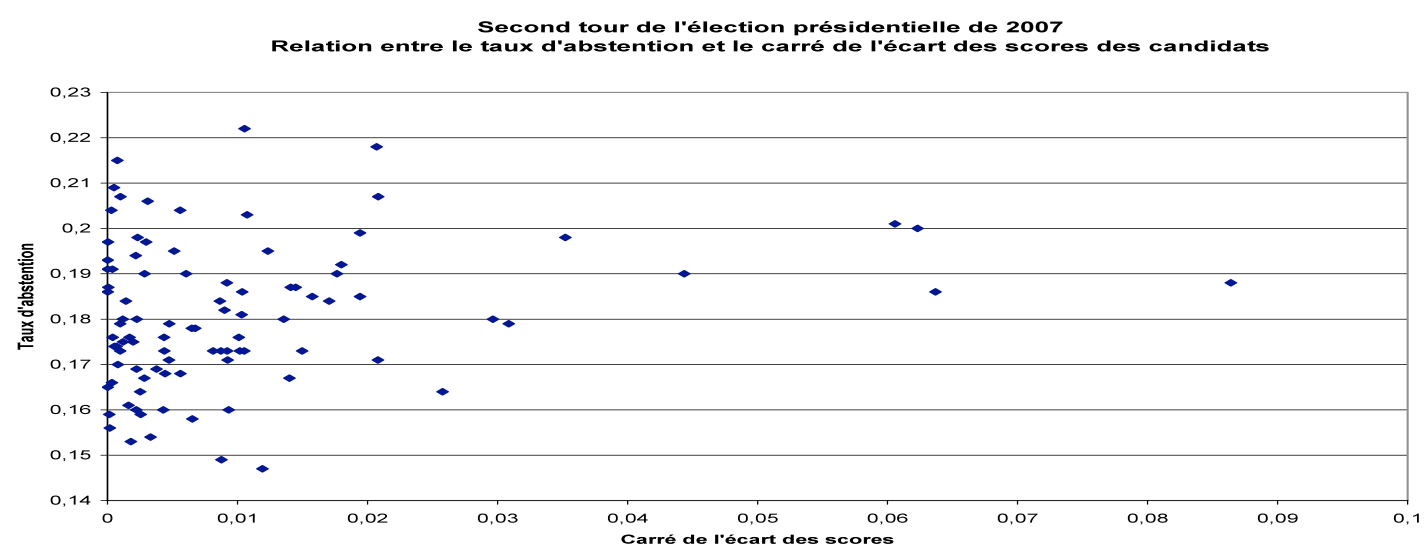

On constate d'abord que le rapport (abstention/indice de lisibilité) est au maximum quand les deux candidats ont un score identique, puis décroît en fonction du carré de l'écart des scores, ce qui permet de ne pas écarter l'hypothèse d'un électorat composé de groupes homogènes :

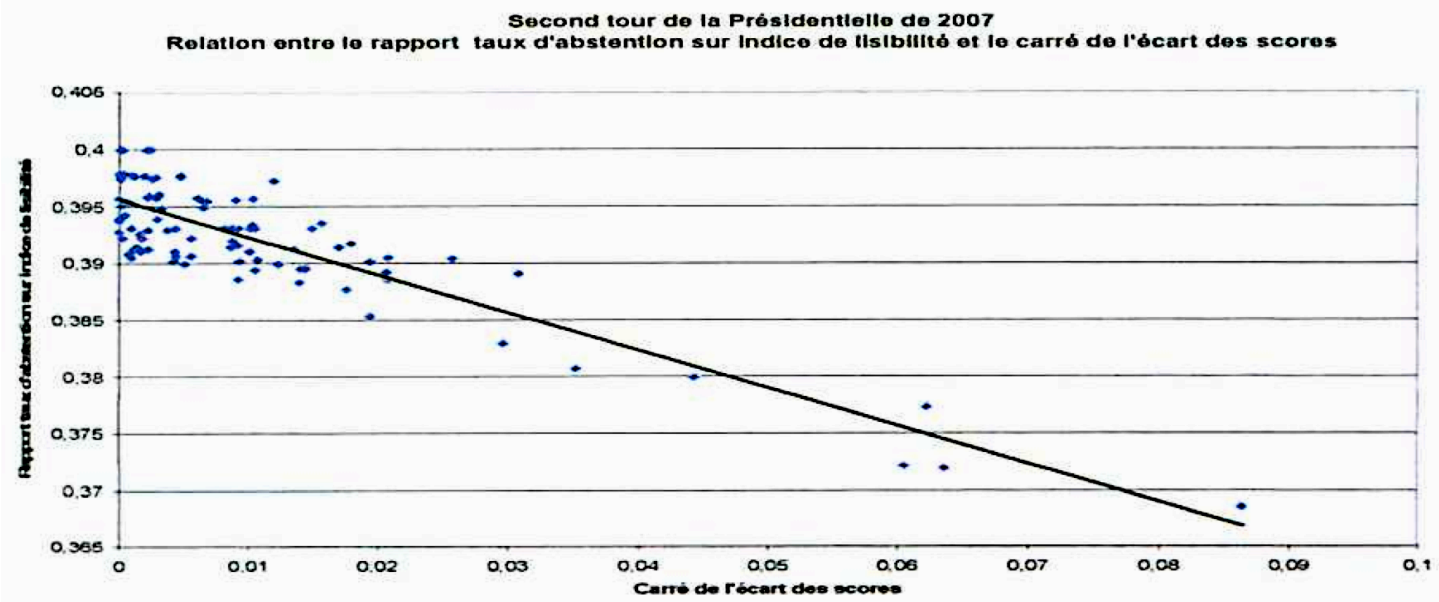


On observe ensuite que le taux d'abstention est pratiquement proportionnel à l'indice de lisibilité, ce qui conduit à penser que l'électorat est composé de deux groupes dont les indices de lisibilité sont significativement différents.

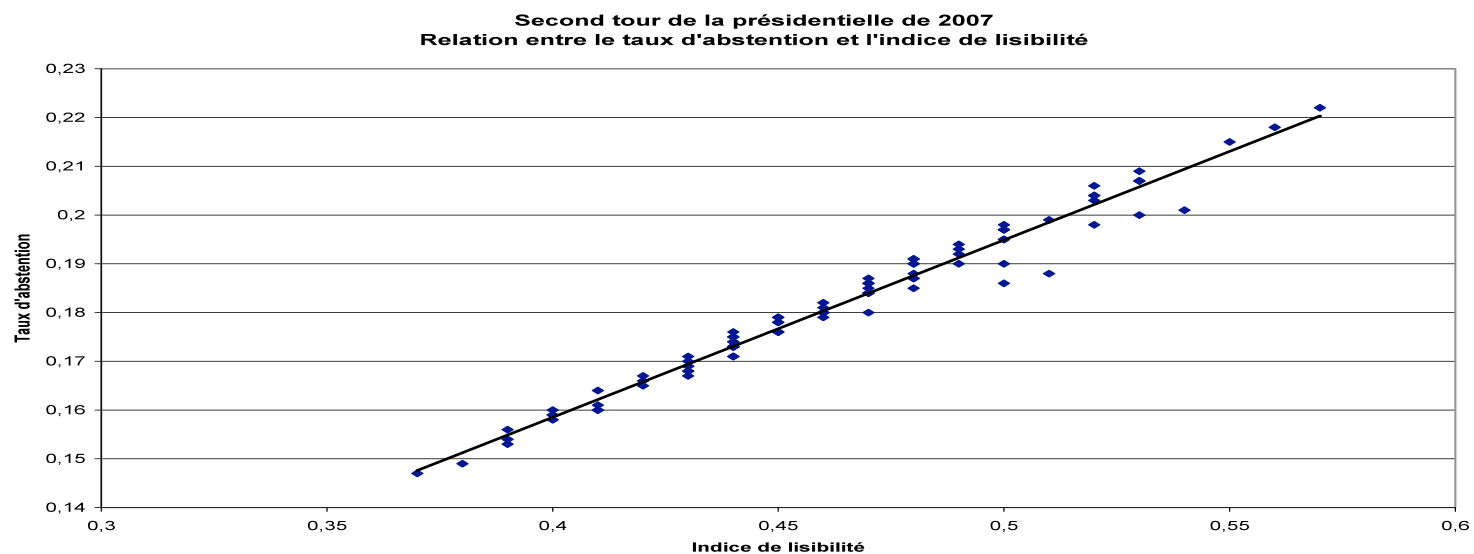

On explore alors l'hypothèse la plus simple : celle d'une partition en deux groupes homogènes, recoupant les électorats des deux candidats, c'est-à-dire celle d'une relation linéaire entre l'indice de lisibilité et la différence de score entre les candidats.

La représentation obtenue à partir de l'ensemble des départements fait apparaître une tendance significative compte tenu de l'aléa lié à l'abstention structurelle.

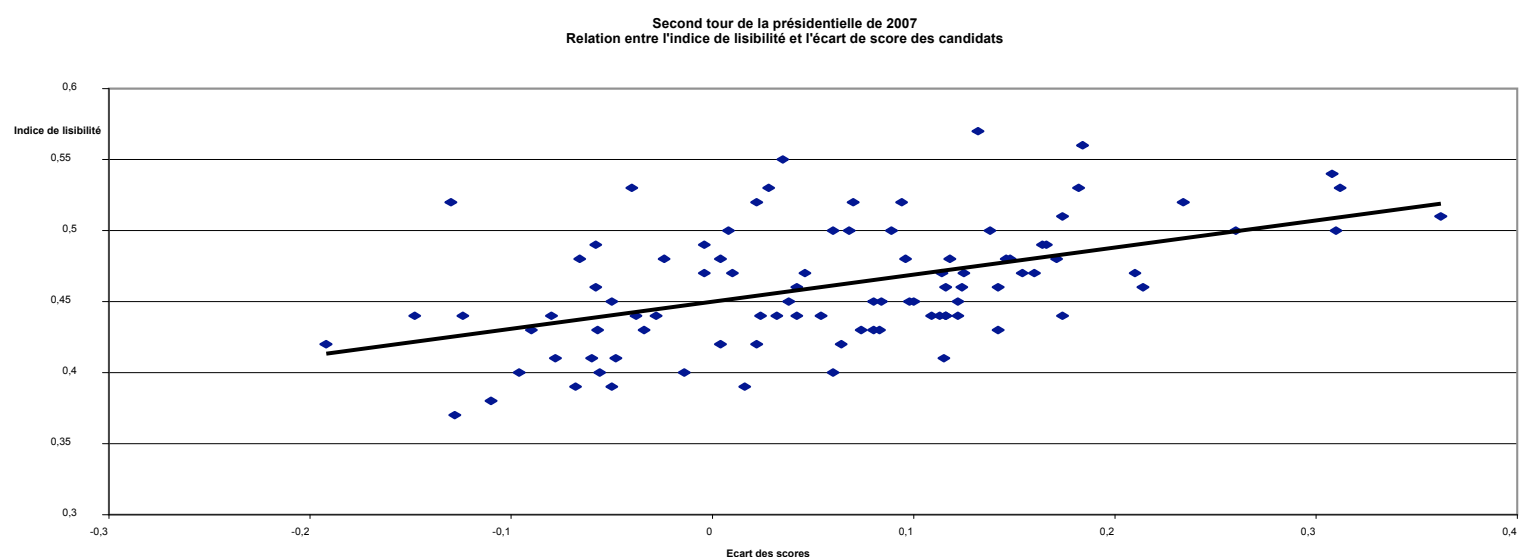

Cette tendance est confirmée par les résultats moyens obtenus sur des groupes de 10 départements, classés en fonction de l'écart des scores obtenus par les deux candidats de manière à réduire l'aléa dû à l'abstention structurelle. Elle apparaît d'autant plus nettement que l'écart des scores est plus marqué.

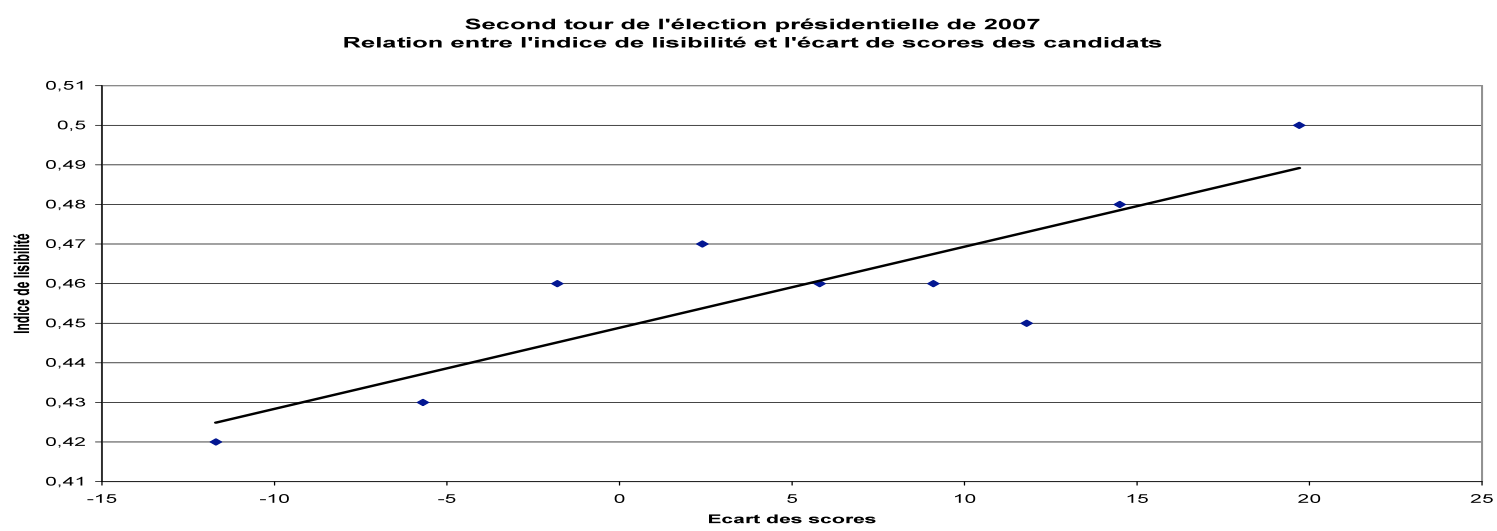


- La comparaison des résultats du référendum de 2005 sur le projet de constitution européenne, et des élections européennes de 2004 fournit un autre exemple de partition de l'électorat en groupes homogènes

L'observation des résultats du référendum de 2005 montre que l'hétérogénéité de l'électorat ne correspond pas à une partition entre ceux qui ont voté oui et ceux qui ont voté non, mais entre ceux qui avaient voté en 2004 et ceux qui s'étaient abstenus. Les variations de l'indice de lisibilité apparaissent en effet sans lien avec les scores du oui et du non, mais en nette corrélation avec les taux d'abstention enregistrés l'année précédente.

Cette caractéristique traduit un double phénomène : la partition au sein de l'électorat sur les questions européennes concerne moins les options proposées que l'implication dans le débat; la mobilisation en 2005 des électeurs qui s'était abstenus en 2004 a été relativement forte. et du non.

Le premier tableau montre que l'indice de lisibilité est pratiquement indépendant des scores du oui

\begin{tabular}{|l|c|c|c|c|}
\hline \multicolumn{4}{|c|}{ Référendum de 2005} & \multicolumn{3}{c|}{ France métropolitaine } \\
\hline \multirow{4}{*}{$\begin{array}{l}\text { Moyennes } \\
\text { calculées sur } \\
\text { des groupes de } \\
\text { dix } \\
\text { départements } \\
\text { classés en } \\
\text { fonction de la }\end{array}$} & \% Oui/inscrits & \% Non/inscrits & Ecart \% & Lisibilité \\
\cline { 2 - 5 } $\begin{array}{l}\text { valeur de } \\
\text { l'indice de } \\
\text { lisibilité }\end{array}$ & 30,5 & 41,7 & $\mathbf{- 1 1 , 2}$ & $\mathbf{0 , 7 2}$ \\
\cline { 2 - 5 } & 28,5 & 42,9 & $\mathbf{- 1 4 , 4}$ & $\mathbf{0 , 7 5}$ \\
\cline { 2 - 5 } & 29,3 & 40,3 & $\mathbf{- 1 0}$ & $\mathbf{0 , 7 6}$ \\
\cline { 2 - 5 } & 27,6 & 40,9 & $\mathbf{- 1 1 , 6}$ & $\mathbf{0 , 7 8}$ \\
\cline { 2 - 5 } & 28,6 & 41,8 & $\mathbf{- 1 4 , 2}$ & $\mathbf{0 , 8}$ \\
\cline { 2 - 5 } & 28,3 & 36,8 & $\mathbf{- 1 3 , 2}$ & $\mathbf{0 , 8 3}$ \\
\cline { 2 - 5 } & 27,6 & 38,2 & $\mathbf{- 6}$ & $\mathbf{0 , 8 6}$ \\
\hline
\end{tabular}

Le deuxième tableau, en revanche met en évidence une très forte corrélation entre le taux d'abstention en 2004 et l'indice de lisibilité de 2005.

\begin{tabular}{|l|c|c|}
\hline \multirow{3}{*}{ en fonction de la proportion d'abstentionnistes en 2004 } \\
\hline & Abstention 2004 & Lisibilité 2005 \\
\cline { 2 - 3 } & $50 \%$ & $\mathbf{0 , 7 1}$ \\
\cline { 2 - 3 } & $53 \%$ & $\mathbf{0 , 7 5}$ \\
\cline { 2 - 3 } & $54 \%$ & $\mathbf{0 , 7 5}$ \\
\cline { 2 - 3 } $\begin{array}{l}\text { Moyennes calculées } \\
\text { sur des groupes de } \\
\text { dix départements } \\
\text { classés en fonction } \\
\text { du taux d'abstention }\end{array}$ enregistré en 2004 & $55 \%$ & $\mathbf{0 , 7 7}$ \\
\cline { 2 - 3 } & $56 \%$ & $\mathbf{0 , 7 9}$ \\
\cline { 2 - 3 } & $57 \%$ & $\mathbf{0 , 8 1}$ \\
\cline { 2 - 3 } & $57,5 \%$ & $\mathbf{0 , 8}$ \\
\cline { 2 - 3 } & $59 \%$ & $\mathbf{0 , 8 3}$ \\
\hline
\end{tabular}


Sur le plan théorique la correspondance qui vient d'être décrite renvoie à l'analyse générale suivante :

- L'électorat est supposé constitué de deux groupes (1) et (2), dont les poids relatifs $\mathrm{q}_{1}$ et $\mathrm{q}_{2}$ sont restés pratiquement inchangés dans les différentes circonscriptions étudiées.

Notant $q=1 / 2\left(q_{1}-q_{2}\right), \bar{q}$ sa valeur moyenne.

- On suppose que, dans la première élection, les deux groupes en question se distinguent notamment par des taux d'abstention nettement différents, $\mathrm{N}_{=1}$ et $\mathrm{N}_{=2}$.

Dans chacune des circonscriptions, le taux d'abstention observé, $\mathrm{N}_{=}$, est estimé par :

$$
\mathrm{N}_{=}=\overline{\mathrm{N}}=+\left[(\overline{\mathrm{q}}-\mathrm{q})\left(\mathrm{N}_{=1}-\mathrm{N}_{=2}\right)\right]
$$

soit $:(\bar{q}-q) \cong\left(N_{=}-\bar{N}_{=}\right) /\left(N_{=1}-N_{=2}\right)$.

Les variations de $\left(\mathrm{N}_{=1}-\mathrm{N}_{=2}\right)$ sont supposées faibles : $(\overline{\mathrm{q}}-\mathrm{q})$ est pratiquement proportionnel à $\left(\mathrm{N}_{=}-\overline{\mathrm{N}}_{=}\right)$

- Les indices de lisibilité I' de la deuxième élection s'expriment par :

$$
I^{\prime} \cong \overline{I^{\prime}}+\left[(\bar{q}-q)\left(I^{\prime}{ }_{1}-I^{\prime}{ }_{2}\right)\right]
$$

Si la partition de l'électorat est demeurée inchangée, il doit exister une corrélation significative entre les taux d'abstention observés d'une circonscription à l'autre dans la première élection, et les indices de lisibilité observés dans la seconde.

Inversement, si on observe des corrélations de cette nature, on peut retenir comme vraisemblable que la partition entre les groupes constituant l'électorat est demeurée inchangée.

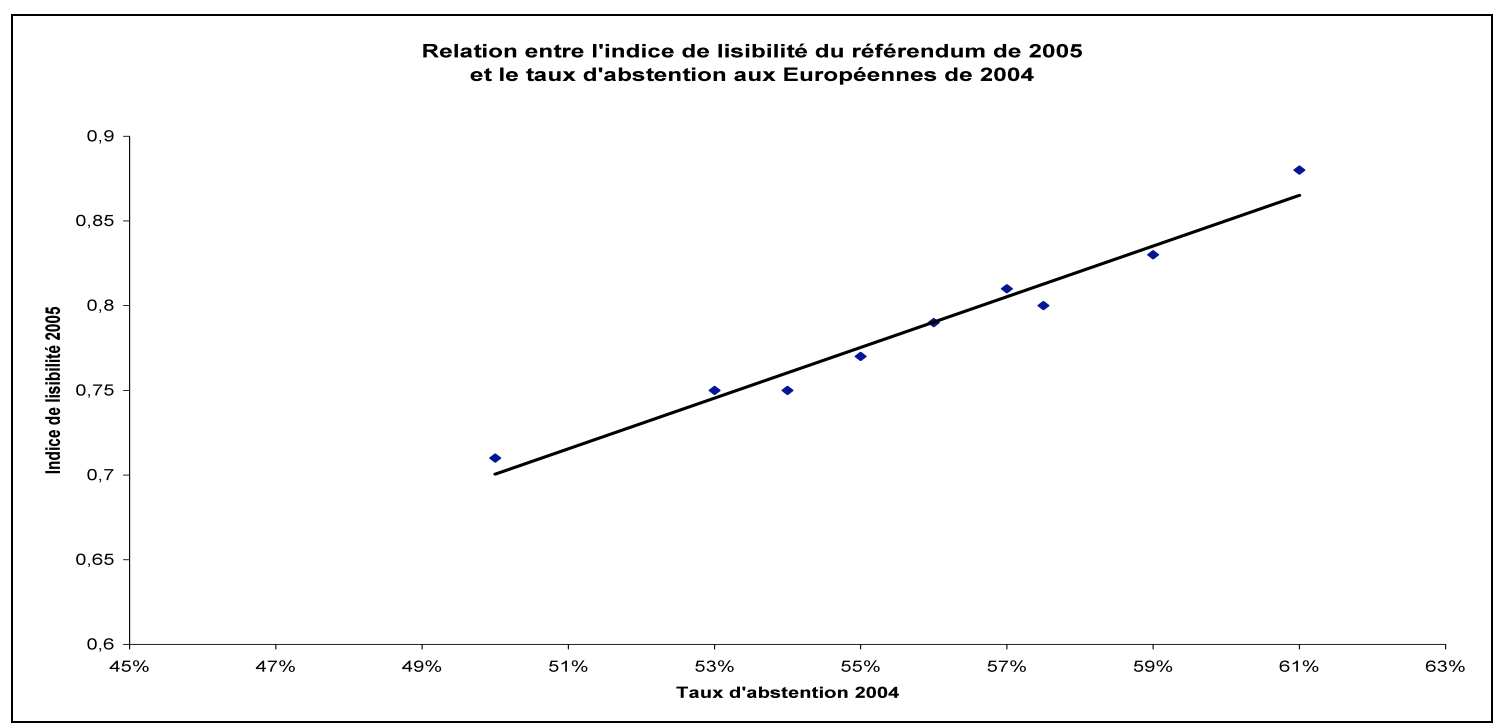

Le coefficient de corrélation entre le taux d'abstention enregistré lors des élections européennes de 2004 et l'indice de lisibilité observé en 2005 est de : 0,92

- Plus généralement, compte tenu de la relation entre indices de lisibilité et taux d'abstention, si la répartition de l'électorat entre (1) et (2) est restée pratiquement inchangée, il doit exister une corrélation significative entre le taux d'abstention observé et celui qui avait été constaté lors de l'élection précédente.

C'est effectivement ce que l'on constate en comparant les résultats des élections européennes de 2004 et ceux du référendum de 2005. 


\section{DÉTAIL DES DONNÉES CHIFFRÉES UTILISÉES ET INDICES DE LISIBILITÉ}

DEUXIÈME TOUR DE L'ÉLECTION PRÉSIDENTIELLE DE 2007

\begin{tabular}{|c|c|c|c|c|}
\hline & \% Sarkozy & \% Royal & $\%$ Abstentions & Lisibilité \\
\hline Ain & 60,5 & 39,5 & 18 & 0,47 \\
\hline Aisne & 53,4 & 46,6 & 19,7 & 0,5 \\
\hline Allier & 48,8 & 51,2 & 19,1 & 0,48 \\
\hline Alpes-de-Haute-Provence & 53,2 & 46,8 & 16,7 & 0,42 \\
\hline Alpes-Maritimes & 68,1 & 31,9 & 18,8 & 0,51 \\
\hline Ardèche & 51,6 & 48,4 & 17,4 & 0,44 \\
\hline Ardennes & 53,5 & 46,5 & 20,6 & 0,52 \\
\hline Ariège & 40,4 & 59,6 & 16,4 & 0,42 \\
\hline Aube & 61,7 & 38,3 & 19,8 & 0,52 \\
\hline Aude & 48,1 & 51,9 & 17,3 & 0,44 \\
\hline Aveyron & 50,8 & 49,2 & 15,6 & 0,39 \\
\hline Bas-Rhin & 65,6 & 34,4 & 20 & 0,53 \\
\hline Bouches-du-Rhône & 58 & 42 & 18,4 & 0,47 \\
\hline Calvados & 51,2 & 48,8 & 17,6 & 0,44 \\
\hline Cantal & 55,8 & 44,2 & 17,1 & 0,44 \\
\hline Charente & 47,1 & 52,9 & 18 & 0,46 \\
\hline Charente-Maritime & 51,9 & 48,1 & 17,9 & 0,45 \\
\hline Cher & 51,4 & 48,6 & 20,9 & 0,53 \\
\hline Corrèze & 47 & 53 & 16,4 & 0,41 \\
\hline Corse-du-Sud & 61,7 & 38,3 & 24,8 & 0,65 \\
\hline Côte d'Armor & 44,5 & 55,5 & 14,9 & 0,38 \\
\hline Côte-d'Or & 56,1 & 43,9 & 17,3 & 0,44 \\
\hline Creuse & 46,7 & 53,3 & 19 & 0,48 \\
\hline Deux-Sèvres & 45,2 & 54,8 & 15,8 & 0,4 \\
\hline Dordogne & 46,6 & 53,4 & 15,4 & 0,39 \\
\hline Doubs & 55,8 & 44,2 & 17,3 & 0,44 \\
\hline Drome & 54,9 & 45,1 & 17,8 & 0,45 \\
\hline Essonne & 52,1 & 47,9 & 17,5 & 0,44 \\
\hline Eure & 57,4 & 42,6 & 18,7 & 0,48 \\
\hline Eure-et-Loir & 58,2 & 41,8 & 19 & 0,49 \\
\hline Finistère & 46,1 & 53,9 & 16 & 0,41 \\
\hline Gard & 56,1 & 43,9 & 17,6 & 0,45 \\
\hline Gers & 47,5 & 52,5 & 15,3 & 0,39 \\
\hline Gironde & 48,3 & 51,7 & 17 & 0,43 \\
\hline Haut-Rhin & 65,4 & 34,6 & 20,1 & 0,54 \\
\hline Haute-Corse & 58,8 & 41,2 & 24,1 & 0,62 \\
\hline Haute-Garonne & 45,5 & 54,5 & 16,8 & 0,43 \\
\hline Haute-Loire & 54,2 & 45,8 & 17,9 & 0,45 \\
\hline Haute-Marne & 59,1 & 40,9 & 20,7 & 0,53 \\
\hline Haute-Saône & 55,8 & 44,2 & 18,2 & 0,46 \\
\hline Haute-Savoie & 63 & 37 & 19 & 0,5 \\
\hline Haute-Vienne & 43,8 & 56,2 & 17,3 & 0,44 \\
\hline Hautes-Alpes & 53,7 & 46,3 & 16,9 & 0,43 \\
\hline Hautes-Pyrénées & 42,6 & 57,4 & 17,3 & 0,44 \\
\hline Hauts-de-Seine & 55,7 & 44,4 & 17,3 & 0,44 \\
\hline Hérault & 54 & 46 & 17,6 & 0,45 \\
\hline Ille-et-Vilaine & 47,6 & 52,4 & 16,1 & 0,41 \\
\hline Indre & 49,8 & 50,2 & 19,3 & 0,49 \\
\hline Indre-et-Loire & 52,7 & 47,3 & 17,5 & 0,44 \\
\hline Isère & 52,1 & 47,9 & 18 & 0,46 \\
\hline Jura & 55 & 45 & 17,8 & 0,45 \\
\hline
\end{tabular}




\begin{tabular}{|c|c|c|c|c|}
\hline Landes & 47,2 & 52,8 & 16 & 0,4 \\
\hline Loir-et-Cher & 55,9 & 44,1 & 18,8 & 0,48 \\
\hline Loire & 54,5 & 45,6 & 19,5 & 0,5 \\
\hline Loire-Atlantique & 47,2 & 52,9 & 16,9 & 0,43 \\
\hline Loiret & 58,6 & 41,5 & 18,5 & 0,48 \\
\hline Lot & 43,6 & 56,4 & 14,7 & 0,37 \\
\hline Lot-et-Garonne & 54 & 46 & 16,8 & 0,43 \\
\hline Lozère & 55,8 & 44,3 & 16 & 0,41 \\
\hline Maine-et-Loire & 54,2 & 45,9 & 17,1 & 0,43 \\
\hline Manche & 56,2 & 43,8 & 18,1 & 0,46 \\
\hline Marne & 59,2 & 40,8 & 21,8 & 0,56 \\
\hline Mayenne & 55,5 & 44,6 & 17,3 & 0,44 \\
\hline Meurthe-et-Moselle & 51,1 & 48,9 & 20,4 & 0,52 \\
\hline Meuse & 56,9 & 43,1 & 19,5 & 0,5 \\
\hline Morbihan & 51,1 & 48,9 & 16,6 & 0,42 \\
\hline Moselle & 56,6 & 43,4 & 22,2 & 0,57 \\
\hline Nièvre & 47,1 & 52,9 & 19,4 & 0,49 \\
\hline Nord & 51,8 & 48,3 & 21,5 & 0,55 \\
\hline Oise & 58,3 & 41,7 & 19,2 & 0,49 \\
\hline Orne & 57,7 & 42,3 & 18,5 & 0,47 \\
\hline Paris & 50,2 & 49,8 & 16,5 & 0,42 \\
\hline Pas-de-Calais & 48 & 52 & 20,7 & 0,53 \\
\hline Puy-de-Dôme & 46 & 54 & 17,3 & 0,44 \\
\hline Pyrénées-Atlantiques & 47,5 & 52,5 & 17,6 & 0,45 \\
\hline Pyrénées-Orientales & 55,7 & 44,3 & 18,4 & 0,47 \\
\hline Rhône & 57,1 & 42,9 & 18 & 0,46 \\
\hline Saône-et-Loire & 53 & 47 & 19,8 & 0,5 \\
\hline Sarthe & 50,4 & 49,6 & 19,7 & 0,5 \\
\hline Savoie & 57,3 & 42,7 & 18,7 & 0,48 \\
\hline Seine-et-Marne & 56,3 & 43,8 & 18,6 & 0,47 \\
\hline Seine-Maritime & 50,2 & 49,8 & 19,1 & 0,48 \\
\hline Seine-Saint-Denis & 43,5 & 56,5 & 20,3 & 0,52 \\
\hline Somme & 50,5 & 49,5 & 18,7 & 0,47 \\
\hline Tarn & 49,3 & 50,7 & 15,9 & 0,4 \\
\hline Tarn-et-Garonne & 53 & 47 & 15,9 & 0,4 \\
\hline Territoire de Belfort & 54,8 & 45,2 & 19 & 0,48 \\
\hline Val-d'Oise & 52,3 & 47,7 & 18,4 & 0,47 \\
\hline Val-de-Marne & 49,8 & 50,2 & 18,6 & 0,47 \\
\hline Var & 65,5 & 34,5 & 18,6 & 0,5 \\
\hline Vaucluse & 60,7 & 39,3 & 17,9 & 0,46 \\
\hline Vendée & 57,1 & 42,9 & 16,7 & 0,43 \\
\hline Vienne & 48,6 & 51,4 & 17,4 & 0,44 \\
\hline Vosges & 54,7 & 45,3 & 20,4 & 0,52 \\
\hline Yonne & 58,7 & 41,3 & 19,9 & 0,51 \\
\hline Yvelines & 58,7 & 41,3 & 17,1 & 0,44 \\
\hline
\end{tabular}

\begin{tabular}{|c|c|}
\hline \multicolumn{2}{|c|}{ Indice de lisibilité 2007} \\
\hline Moyenne & 0,46 \\
\hline Coefficient de variabilité & 0,106 \\
\hline
\end{tabular}


DEUXIÈMES TOURS DES ÉLECTIONS PRÉSIDENTIELLES DE 1995 ET 2002

\begin{tabular}{|c|c|c|c|c|c|c|c|c|}
\hline & \multicolumn{4}{|c|}{1995} & \multicolumn{4}{|c|}{2002} \\
\hline & Chirac & Jospin & Abstentions & Lisibilité & Chirac & Le Pen & Abstentions & Lisibilité \\
\hline Ain & 57,43 & 42,57 & 19,89 & 0,51 & 78,7 & 21,3 & 23,4 & 0,72 \\
\hline Aisne & 45,45 & 54,55 & 17,96 & 0,46 & 75,4 & 24,6 & 24,9 & $\mathbf{0 , 7 3}$ \\
\hline Allier & 49,74 & 50,26 & 18,4 & 0,47 & 85,2 & 14,8 & 24,6 & $\mathbf{0 , 8 5}$ \\
\hline Alpes-de-Hte-Provence & 52,08 & 47,92 & 17,68 & 0,62 & 78,8 & 21,2 & 22,6 & 0,7 \\
\hline Hautes-Alpes & 54,97 & 45,03 & 17,92 & 0,45 & 82,5 & 17,5 & 22,5 & 0,74 \\
\hline Alpes-Maritimes & 65,48 & 34,52 & 23,24 & 0,43 & 71,3 & 28,7 & 27,4 & $\mathbf{0 , 7 7}$ \\
\hline Ardèche & 51,63 & 48,37 & 17,02 & 0,5 & 82,1 & 17,9 & 22,3 & $\mathbf{0 , 7 3}$ \\
\hline Ardennes & 46,71 & 53,29 & 19,79 & 0,42 & 75,9 & 24,1 & 26,9 & 0,79 \\
\hline Ariège & 40,17 & 59,83 & 16,22 & 0,47 & 83,9 & 16,1 & 24,1 & 0,82 \\
\hline Aube & 55,22 & 44,78 & 18,57 & 0,39 & 76,8 & 23,2 & 24,5 & $\mathbf{0 , 7 3}$ \\
\hline Aude & 44,34 & 55,66 & 15,51 & 0,38 & 77,9 & 22,1 & 24,3 & 0,74 \\
\hline Aveyron & 55,87 & 44,13 & 14,8 & 0,58 & 88,2 & 11,8 & 20,6 & $\mathbf{0 , 7 9}$ \\
\hline Bouches-du-Rhône & 54,46 & 45,54 & 22,35 & 0,57 & 72,6 & 27,4 & 23,1 & 0,66 \\
\hline Calvados & 51,11 & 48,89 & 18,9 & 0,48 & 84 & 16 & 23,1 & 0,79 \\
\hline Cantal & 62,94 & 37,06 & 15,64 & 0,41 & 88,5 & 11,5 & 21,5 & 0,83 \\
\hline Charente & 46,97 & 53,03 & 19,39 & 0,49 & 85,2 & 14,8 & 24,1 & 0,84 \\
\hline Charente-Maritime & 51,67 & 48,33 & 19,53 & 0,49 & 83,7 & 16,3 & 23,3 & 0,79 \\
\hline Cher & 50,65 & 49,35 & 20,24 & 0,51 & 83,1 & 16,9 & 24,8 & 0,82 \\
\hline Corrèze & 61,37 & 38,63 & 12,43 & 0,32 & 90,9 & 9,1 & 19,4 & 0,83 \\
\hline Corse-du-Sud & 62,13 & 37,87 & 25,06 & 0,66 & 77,7 & 22,3 & 35,1 & 1,05 \\
\hline Haute-Corse & 57,23 & 42,77 & 21,87 & 0,38 & 81,6 & 18,4 & 39 & 1,22 \\
\hline Côte-d'Or & 54,19 & 45,81 & 18,9 & 0,48 & 81,7 & 18,3 & 22,9 & 0,75 \\
\hline Côte d'Armor & 45,92 & 54,08 & 15,11 & 0,45 & 88,3 & 11,7 & 20,7 & 0,8 \\
\hline Creuse & 53,64 & 46,36 & 17,7 & 0,45 & 88,3 & 11,7 & 24 & 0,9 \\
\hline Dordogne & 50,02 & 49,98 & 14,31 & 0,36 & 85,7 & 14,3 & 21,3 & 0,76 \\
\hline Doubs & 52,41 & 47,59 & 16,88 & 0,43 & 81,9 & 18,1 & 22,3 & $\mathbf{0 , 7 3}$ \\
\hline Drôme & 52,82 & 47,18 & 18,66 & 0,47 & 79,3 & 20,7 & 22,6 & 0,71 \\
\hline Eure & 52,97 & 47,03 & 18,98 & 0,49 & 78,3 & 21,7 & 23,6 & 0,72 \\
\hline Eure-et-Loir & 54,27 & 45,73 & 19,58 & 0,48 & 79,3 & 20,7 & 23,7 & 0,74 \\
\hline Finistère & 51,16 & 48,84 & 17,13 & 0,5 & 89,4 & 10,6 & 21,4 & 0,85 \\
\hline Gard & 51,07 & 48,93 & 18,73 & 0,43 & 73,4 & 26,6 & 23,8 & 0,68 \\
\hline Haute-Garonne & 45,53 & 54,47 & 18,55 & 0,47 & 84,1 & 15,9 & 22,4 & 0,77 \\
\hline Gers & 49,22 & 50,78 & 15,34 & 0,39 & 85,2 & 14,8 & 21 & 0,74 \\
\hline Gironde & 48,79 & 51,21 & 18,14 & 0,46 & 83,8 & 16,2 & 22,8 & 0,77 \\
\hline Hérault & 51,08 & 48,92 & 18,14 & 0,56 & 75,5 & 24,5 & 24 & 0,71 \\
\hline Ille-et-Vilaine & 51,19 & 48,81 & 18,48 & 0,47 & 89,8 & 10,2 & 20,8 & 0,84 \\
\hline Indre & 49,7 & 50,3 & 17,91 & 0,43 & 83,3 & 16,7 & 23,3 & $\mathbf{0 , 7 8}$ \\
\hline Indre-et-Loire & 50,58 & 49,42 & 20,15 & 0,51 & 84,9 & 15,1 & 23,2 & 0,8 \\
\hline Isère & 49,83 & 50,17 & 20,54 & 0,46 & 81,7 & 18,3 & 22,4 & $\mathbf{0 , 7 3}$ \\
\hline Jura & 52,29 & 47,71 & 17,32 & 0,51 & 81,1 & 18,9 & 23,3 & 0,75 \\
\hline Landes & 47,29 & 52,71 & 15,07 & 0,54 & 87,1 & 12,9 & 22,4 & 0,83 \\
\hline Loir-et-Cher & 51,8 & 48,2 & 18,24 & 0,47 & 80,8 & 19,2 & 23 & $\mathbf{0 , 7 3}$ \\
\hline Loire & 55,46 & 44,54 & 22,16 & 0,41 & 79,4 & 20,6 & 24,7 & 0,77 \\
\hline Haute-Loire & 57,75 & 42,25 & 16,96 & 0,55 & 81,8 & 18,2 & 22,7 & 0,74 \\
\hline Loire-Atlantique & 50,33 & 49,67 & 20,24 & 0,56 & 88,8 & 11,2 & 22,4 & $\mathbf{0 , 8 7}$ \\
\hline Loiret & 56,1 & 43,9 & 18,65 & 0,46 & 81,3 & 18,7 & 22,5 & $\mathbf{0 , 7 3}$ \\
\hline Lot & 48,11 & 51,89 & 13,25 & 0,47 & 87,9 & 12,1 & 20,4 & $\mathbf{0 , 7 8}$ \\
\hline Lot-et-Garonne & 52,06 & 47,94 & 15,7 & 0,45 & 77,9 & 22,1 & 22,5 & 0,69 \\
\hline Lozère & 61,45 & 38,55 & 14,39 & 0,51 & 85,6 & 14,4 & 21,1 & 0,75 \\
\hline Maine-et-Loire & 56,99 & 43,01 & 18,47 & 0,52 & 87,9 & 12,1 & 21,2 & 0,81 \\
\hline Manche & 58,87 & 41,13 & 18,38 & 0,44 & 85,3 & 14,7 & 22,6 & 0,79 \\
\hline
\end{tabular}




\begin{tabular}{|c|c|c|c|c|c|c|c|c|}
\hline Marne & 54,53 & 45,47 & 21,49 & 0,38 & 79,9 & 20,1 & 26,4 & 0,82 \\
\hline Haute-Marne & 52,18 & 47,82 & 19,98 & 0,57 & 76,2 & 23,8 & 26,3 & 0,78 \\
\hline Mayenne & 59,49 & 40,51 & 17,78 & 0,51 & 88,5 & 11,5 & 21,4 & $\mathbf{0 , 8 3}$ \\
\hline Meurthe-et-Moselle & 48,01 & 51,99 & 20,81 & $\mathbf{0 , 4 8}$ & 81,7 & 18,3 & 25,1 & $\mathbf{0 , 8 1}$ \\
\hline Meuse & 52,29 & 47,71 & 18,49 & 0,46 & 78,2 & 21,8 & 24,6 & 0,75 \\
\hline Morbihan & 53,45 & 46,55 & 17,26 & $\mathbf{0 , 3 3}$ & 86,1 & 13,9 & 21,6 & $\mathbf{0 , 7 8}$ \\
\hline Moselle & 51,3 & 48,7 & 21,81 & 0,4 & 78,1 & 21,9 & 26,5 & 0,8 \\
\hline Nièvre & 42,93 & 57,07 & 18,24 & 0,37 & 81,7 & 18,3 & 26,7 & $\mathbf{0 , 8 6}$ \\
\hline Nord & 46,29 & 53,71 & 21,08 & 0,47 & 78,3 & 21,7 & 26,6 & $\mathbf{0 , 8 1}$ \\
\hline Oise & 51,12 & 48,88 & 18,91 & 0,47 & 74,9 & 25,1 & 24,4 & $\mathbf{0 , 7 1}$ \\
\hline Orne & 57,61 & 42,39 & 17,73 & 0,55 & 81,3 & 18,7 & 22 & $\mathbf{0 , 7 1}$ \\
\hline Pas-de-Calais & 42,72 & 57,28 & 18,89 & 0,46 & 77,9 & 22,1 & 26,4 & 0,8 \\
\hline Puy-de-Dôme & 51,12 & 48,88 & 17,17 & $\mathbf{0 , 5 3}$ & 87,1 & 12,9 & 22,5 & $\mathbf{0 , 8 3}$ \\
\hline Pyrénées-Atlantiques & 53,9 & 46,1 & 17,54 & 0,47 & 87,5 & 12,5 & 22,8 & 0,84 \\
\hline Hautes-Pyrénées & 46,58 & 53,42 & 18,38 & 0,44 & 86,6 & 13,4 & 23,1 & $\mathbf{0 , 8 4}$ \\
\hline Pyrénées-Orientales & 51,77 & 48,23 & 18,88 & 0,55 & 74,9 & 25,1 & 25 & $\mathbf{0 , 7 3}$ \\
\hline Bas-Rhin & 58,97 & 41,03 & 22,29 & 0,47 & 79,4 & 20,6 & 24,4 & 0,76 \\
\hline Haut-Rhin & 57,26 & 42,74 & 21,25 & 0,54 & 77,6 & 22,4 & 24,5 & $\mathbf{0 , 7 4}$ \\
\hline Rhône & 57,93 & 42,07 & 15,66 & 0,48 & 81,3 & 18,7 & 22,5 & $\mathbf{0 , 7 3}$ \\
\hline Haute-Saône & 50,39 & 49,61 & 20,08 & 0,45 & 75,7 & 24,3 & 25 & $\mathbf{0 , 7 4}$ \\
\hline Saône-et-Loire & 51,38 & 48,62 & 19,95 & 0,59 & 81,9 & 18,1 & 25,9 & $\mathbf{0 , 8 4}$ \\
\hline Sarthe & 49,21 & 50,79 & 19,89 & 0,48 & 84,4 & 15,6 & 24,3 & $\mathbf{0 , 8 3}$ \\
\hline Savoie & 56,07 & 43,93 & 20,33 & 0,43 & 81,3 & 18,7 & 24 & 0,77 \\
\hline Haute-Savoie & 61,96 & 38,04 & 20,83 & 0,44 & 81,8 & 18,2 & 23,8 & $\mathbf{0 , 7 7}$ \\
\hline Paris & 60,17 & 39,83 & 22,57 & 0,48 & 90 & 10 & 19,3 & 0,8 \\
\hline Seine-Maritime & 46,77 & 53,23 & 20,83 & 0,48 & 82,5 & 17,5 & 23,8 & $\mathbf{0 , 7 8}$ \\
\hline Seine-et-Marne & 55,5 & 44,5 & 20,68 & 0,51 & 80,6 & 19,4 & 23,6 & 0,75 \\
\hline Yvelines & 60,64 & 39,36 & 18,62 & $\mathbf{0 , 5}$ & 85,6 & 14,4 & 21,2 & $\mathbf{0 , 7 5}$ \\
\hline Deux-Sèvres & 52,49 & 47,51 & 17,69 & $\mathbf{0 , 5 2}$ & 89,6 & 10,4 & 20,3 & $\mathbf{0 , 8 2}$ \\
\hline Somme & 46,87 & 53,13 & 16,61 & 0,53 & 78,3 & 21,7 & 24,1 & 0,74 \\
\hline Tarn & 49,44 & 50,56 & 15,22 & 0,53 & 82,4 & 17,6 & 21,3 & 0,71 \\
\hline Tarn-et-Garonne & 50,92 & 49,08 & 15,55 & 0,65 & 78,4 & 21,6 & 21,5 & 0,67 \\
\hline Var & 61,59 & 38,41 & 21,63 & 0,42 & 71,3 & 28,7 & 24,8 & 0,69 \\
\hline Vaucluse & 55,85 & 44,15 & 18,91 & $\mathbf{0 , 3 8}$ & 70,4 & 29,6 & 22,7 & 0,63 \\
\hline Vendée & 60,5 & 39,5 & 16,58 & 0,39 & 87,8 & 12,2 & 20,7 & $\mathbf{0 , 7 9}$ \\
\hline Vienne & 50,33 & 49,67 & 18,51 & 0,43 & 87,7 & 12,3 & 21,2 & 0,8 \\
\hline Haute-Vienne & 48,16 & 51,84 & 16,19 & 0,55 & 88,9 & 11,1 & 21,4 & 0,84 \\
\hline Vosges & 51,43 & 48,57 & 18,13 & $\mathbf{0 , 5 3}$ & 78,8 & 21,2 & 24,5 & $\mathbf{0 , 7 5}$ \\
\hline Yonne & 54,82 & 45,18 & 18,92 & 0,56 & 77,3 & 22,7 & 23,8 & $\mathbf{0 , 7 2}$ \\
\hline Territoire de Belfort & 48,59 & 51,41 & 17,18 & 0,48 & 77,9 & 22,1 & 24,4 & $\mathbf{0 , 7 4}$ \\
\hline Essonne & 53,3 & 46,7 & 19,26 & 0,43 & 84,9 & 15,1 & 22,5 & 0,78 \\
\hline Hauts-de-Seine & 59,02 & 40,98 & 21,56 & 0,47 & 88 & 12 & 21,7 & $\mathbf{0 , 8 2}$ \\
\hline Seine-Saint-Denis & 48,16 & 51,84 & 25,61 & 0,46 & 82,6 & 17,4 & 26,6 & $\mathbf{0 , 8 7}$ \\
\hline Val-de-Marne & 52,99 & 47,01 & 21,55 & 0,48 & 86,2 & 13,8 & 23,2 & $\mathbf{0 , 8 3}$ \\
\hline Val-d'Oise & 53,78 & 46,22 & 20,95 & 0,48 & 82,8 & 17,2 & 23,4 & 0,78 \\
\hline
\end{tabular}

\begin{tabular}{|c|c|c|}
\hline \multicolumn{3}{|c|}{ Indices de lisibilité } \\
\hline & 1995 & 2002 \\
\hline Moyenne & 0,48 & 0,78 \\
\hline Variabilité & 0,131 & 0,096 \\
\hline
\end{tabular}


DEUXIÈMES TOURS DES ÉLECTIONS PRÉSIDENTIELLES DE 1974 ET 1981

\begin{tabular}{|c|c|c|c|c|c|c|c|c|}
\hline & \multicolumn{4}{|c|}{1974} & \multicolumn{4}{|c|}{1981} \\
\hline & Abstention & Giscard & Mitterrand & Lisibilité & Abstentions & Giscard & Mitterrand & Lisibilité \\
\hline Ain & 13,2 & 54,89 & 45,11 & $\mathbf{0 , 3 3 4}$ & 13,32 & 52,22 & 47,78 & $\mathbf{0 , 3 3 6}$ \\
\hline Aisne & 9,99 & 44,36 & 55,64 & 0,253 & 11,38 & 43,31 & 56,69 & 0,289 \\
\hline Allier & 11,31 & 45,52 & 54,48 & 0,286 & 12,78 & 43,95 & 56,05 & $\mathbf{0 , 3 2 5}$ \\
\hline Alpes-de-Hte-Provence & 10,64 & 46,48 & 53,52 & 0,268 & 12,6 & 46,46 & 53,54 & 0,318 \\
\hline Hautes-Alpes & 11,82 & 51,81 & 48,19 & 0,298 & 13,31 & 48,77 & 51,23 & $\mathbf{0 , 3 3 5}$ \\
\hline Alpes-Maritimes & 12,09 & 53,65 & 46,35 & $\mathbf{0 , 3 0 5}$ & 15,72 & 54,37 & 45,63 & $\mathbf{0 , 3 9 8}$ \\
\hline Ardèche & 10,77 & 52,36 & 47,64 & $\mathbf{0 , 2 7 1}$ & 12,9 & 49,38 & 50,62 & $\mathbf{0 , 3 2 5}$ \\
\hline Ardennes & 10,78 & 46,44 & 53,56 & 0,272 & 11,81 & 44,02 & 55,98 & $\mathbf{0 , 3}$ \\
\hline Ariège & 11,35 & 36,36 & 63,64 & $\mathbf{0 , 3}$ & 13,09 & 36,77 & 63,23 & 0,344 \\
\hline Aube & 11,35 & 51,02 & 48,98 & 0,286 & 13,64 & 49,93 & 50,07 & 0,344 \\
\hline Aude & 9,87 & 38,07 & 61,93 & 0,258 & 11,4 & 36,33 & 63,67 & 0,301 \\
\hline Aveyron & 10,39 & 56,61 & 43,39 & 0,264 & 11,31 & 51,88 & 48,12 & 0,285 \\
\hline Bouches-du-Rhône & 15,23 & 43,64 & 56,36 & 0,388 & 17,37 & 43,69 & 56,31 & 0,443 \\
\hline Calvados & 12,09 & 55,58 & 44,42 & $\mathbf{0 , 3 0 7}$ & 13,11 & 49,59 & 50,41 & $\mathbf{0 , 3 3}$ \\
\hline Cantal & 13,61 & 61,49 & 38,51 & 0,354 & 13,84 & 56,93 & 43,07 & $\mathbf{0 , 3 5 3}$ \\
\hline Charente & 11,96 & 45,9 & 54,1 & 0,302 & 12,29 & 43,88 & 56,12 & 0,312 \\
\hline Charente-Maritime & 14,27 & 50,4 & 49,6 & 0,36 & 14,48 & 46,47 & 53,53 & 0,366 \\
\hline Cher & 11,42 & 47,98 & 52,02 & $\mathbf{0 , 2 8 8}$ & 12,4 & 47,18 & 52,82 & 0,313 \\
\hline Corrèze & 9,81 & 44,43 & 55,57 & 0,249 & 11,06 & 40,26 & 59,74 & 0,285 \\
\hline Corse-du-Sud & 24,28 & 55,5 & 44,5 & 0,622 & 25,11 & 54,11 & 45,89 & 0,642 \\
\hline Haute-Corse & 21,47 & 51,37 & 48,63 & 0,545 & 27,67 & 50,51 & 49,49 & $\mathbf{0 , 7 0 8}$ \\
\hline Côte-d'Or & 12,86 & 51,59 & 48,41 & 0,324 & 13,5 & 47,46 & 52,54 & 0,341 \\
\hline Côte d'Armor & 9,61 & 49,77 & 50,23 & 0,241 & 10,07 & 44,46 & 55,54 & 0,255 \\
\hline Creuse & 14,54 & 44,03 & 55,97 & $\mathbf{0 , 3 7}$ & 14,87 & 43,64 & 56,36 & 0,379 \\
\hline Dordogne & 9,62 & 44,48 & 55,52 & 0,244 & 10,88 & 42,1 & 57,9 & 0,278 \\
\hline Doubs & 11,15 & 52,5 & 47,5 & 0,281 & 12,93 & 47,6 & 52,4 & 0,326 \\
\hline Drôme & 11,77 & 48,15 & 51,85 & 0,296 & 13,7 & 45,89 & 54,11 & 0,347 \\
\hline Eure & 10,54 & 50,97 & 49,03 & 0,265 & 11,88 & 48,98 & 51,02 & 0,299 \\
\hline Eure-et-Loir & 10,67 & 52,16 & 47,84 & 0,269 & 12 & 50,79 & 49,21 & 0,302 \\
\hline Finistère & 11,66 & 58,4 & 41,6 & 0,299 & 12,77 & 50,93 & 49,07 & 0,322 \\
\hline Gard & 11,55 & 43,99 & 56,01 & 0,293 & 14,04 & 42,53 & 57,47 & 0,359 \\
\hline Haute-Garonne & 11,93 & 43,64 & 56,36 & $\mathbf{0 , 3 0 3}$ & 13,96 & 39,21 & 60,79 & 0,362 \\
\hline Gers & 11,58 & 42,23 & 57,77 & 0,296 & 11,64 & 40,97 & 59,03 & 0,299 \\
\hline Gironde & 14,52 & 45,42 & 54,58 & 0,368 & 13,69 & 42,27 & 57,73 & 0,35 \\
\hline Hérault & 12,35 & 45,22 & 54,78 & 0,313 & 13,91 & 43,64 & 56,36 & 0,354 \\
\hline Ille-et-Vilaine & 11,88 & 61,77 & 38,23 & $\mathbf{0 , 3 1}$ & 12,03 & 54,18 & 45,82 & 0,304 \\
\hline Indre & 12,22 & 47,93 & 52,07 & 0,308 & 12,25 & 46,7 & 53,3 & 0,309 \\
\hline Indre-et-Loire & 13,11 & 53,08 & 46,92 & $\mathbf{0 , 3 3 1}$ & 13,73 & 47,41 & 52,59 & 0,346 \\
\hline Isère & 12,54 & 46,68 & 53,32 & 0,316 & 13,8 & 44,11 & 55,89 & 0,351 \\
\hline Jura & 11,04 & 50,99 & 49,01 & 0,278 & 11,61 & 47,54 & 52,46 & 0,292 \\
\hline Landes & 10,49 & 46,71 & 53,29 & 0,264 & 10,92 & 43,83 & 56,17 & 0,277 \\
\hline Loir-et-Cher & 10,92 & 51,41 & 48,59 & 0,275 & 11,51 & 49,46 & 50,54 & 0,29 \\
\hline Loire & 14,58 & 52,13 & 47,87 & 0,368 & 15,21 & 48,9 & 51,1 & 0,384 \\
\hline Haute-Loire & 12,06 & 59,69 & 40,31 & 0,311 & 12,36 & 56,02 & 43,98 & 0,314 \\
\hline Loire-Atlantique & 12,9 & 56,97 & 43,03 & 0,329 & 15,13 & 50,09 & 49,91 & 0,382 \\
\hline Loiret & 10,61 & 55,32 & 44,68 & 0,269 & 11,98 & 52,18 & 47,82 & $\mathbf{0 , 3 0 2}$ \\
\hline Lot & 8,97 & 46,08 & 53,92 & 0,226 & 9,89 & 40,42 & 59,58 & 0,255 \\
\hline Lot-et-Garonne & 11,25 & 44,98 & 55,02 & 0,285 & 11,49 & 43,37 & 56,63 & 0,292 \\
\hline Lozère & 13,12 & 64,31 & 35,69 & 0,348 & 13,08 & 40,57 & 59,43 & $\mathbf{0 , 3 3 7}$ \\
\hline Maine-et-Loire & 12,31 & 63,23 & 36,77 & $\mathbf{0 , 3 2 4}$ & 12,82 & 57,64 & 42,36 & $\mathbf{0 , 3 2 7}$ \\
\hline Manche & 12,03 & 65,41 & 34,59 & 0,322 & 13,08 & 59,13 & 40,87 & 0,336 \\
\hline Marne & 12,49 & 50,9 & 49,1 & 0,314 & 13,86 & 50,39 & 49,61 & 0,349 \\
\hline Haute-Marne & 11,73 & 52,3 & 47,7 & 0,295 & 13,07 & 48,02 & 51,98 & 0,329 \\
\hline Mayenne & 10,64 & 66,98 & 33,02 & 0,289 & 11,56 & 60,06 & 39,94 & 0,298 \\
\hline Meurthe-et-Moselle & 12,6 & 49,48 & 50,52 & 0,317 & 14,52 & 45,81 & 54,19 & 0,368 \\
\hline
\end{tabular}




\begin{tabular}{|c|c|c|c|c|c|c|c|c|}
\hline Meuse & 11,79 & 54,1 & 45,9 & 0,298 & 11,83 & 51,16 & 48,84 & 0,298 \\
\hline Morbihan & 12,61 & 62,17 & 37,83 & $\mathbf{0 , 3 3}$ & 12,77 & 53,97 & 46,03 & 0,323 \\
\hline Moselle & 12,58 & 53,97 & 46,03 & 0,318 & 13,9 & 51,05 & 48,95 & 0,35 \\
\hline Nièvre & 11,34 & 38,65 & 61,35 & 0,295 & 12,83 & 37,08 & 62,92 & $\mathbf{0 , 3 3 7}$ \\
\hline Nord & 10,17 & 45,87 & 54,13 & 0,257 & 11,75 & 44,64 & 55,36 & 0,298 \\
\hline Oise & 10,17 & 47,42 & 52,58 & 0,256 & 11,56 & 45,39 & 54,61 & 0,292 \\
\hline Orne & 11,59 & 60,6 & 39,4 & 0,3 & 12,36 & 54,97 & 45,03 & $\mathbf{0 , 3 1 3}$ \\
\hline Pas-de-Calais & 9,42 & 42,09 & 57,91 & 0,241 & 10,78 & 41,79 & 58,21 & 0,276 \\
\hline Puy-de-Dôme & 10,85 & 52,18 & 47,82 & 0,273 & 12,08 & 48,07 & 51,93 & 0,304 \\
\hline Pyrénées-Atlantiques & 12,71 & 56,19 & 43,81 & $\mathbf{0 , 3 2 3}$ & 12,59 & 50,42 & 49,58 & $\mathbf{0 , 3 1 7}$ \\
\hline Hautes-Pyrénées & 12,89 & 43,17 & 56,83 & 0,328 & 14,31 & 39,94 & 60,06 & $\mathbf{0 , 3 7}$ \\
\hline Pyrénées-Orientales & 14,03 & 43,34 & 56,66 & $\mathbf{0 , 3 5 7}$ & 15,43 & 43,69 & 56,31 & $\mathbf{0 , 3 9 3}$ \\
\hline Bas-Rhin & 14,47 & 67,02 & 32,98 & 0,392 & 14,53 & 65,11 & 34,89 & $\mathbf{0 , 3 8 7}$ \\
\hline Haut-Rhin & 13,95 & 65,76 & 34,24 & 0,374 & 14,73 & 59,71 & 40,29 & $\mathbf{0 , 3 8}$ \\
\hline Rhône & 13,15 & 52,7 & 47,3 & 0,332 & 15,66 & 49,29 & 50,71 & 0,395 \\
\hline Haute-Saône & 9,17 & 50,18 & 49,82 & $\mathbf{0 , 2 3}$ & 10,64 & 47,33 & 52,67 & 0,268 \\
\hline Saône-et-Loire & 13,75 & 49,03 & 50,97 & 0,346 & 14,78 & 46,84 & 53,16 & 0,374 \\
\hline Sarthe & 11,53 & 51,39 & 48,61 & $\mathbf{0 , 2 9}$ & 12,6 & 49,3 & 50,7 & $\mathbf{0 , 3 1 7}$ \\
\hline Savoie & 12,59 & 50,63 & 49,37 & 0,317 & 14,99 & 49,55 & 50,45 & 0,378 \\
\hline Haute-Savoie & 13,24 & 59,39 & 40,61 & 0,341 & 15,16 & 55,78 & 44,22 & 0,385 \\
\hline Paris & 14,68 & 56,92 & 43,08 & 0,374 & 17,3 & 53,56 & 46,44 & 0,438 \\
\hline Seine-Maritime & 11,96 & 46,57 & 53,43 & 0,302 & 12,58 & 44,53 & 55,47 & $\mathbf{0 , 3 1 9}$ \\
\hline Seine-et-Marne & 12,48 & 50,7 & 49,3 & 0,314 & 13,85 & 47,36 & 52,64 & $\mathbf{0 , 3 5}$ \\
\hline Yvelines & 11,47 & 53,14 & 46,86 & 0,289 & 13,5 & 51,09 & 48,91 & 0,34 \\
\hline Deux-Sèvres & 11,48 & 57,72 & 42,28 & 0,293 & 11,59 & 52,43 & 47,57 & 0,292 \\
\hline Somme & 8,22 & 45,63 & 54,37 & $\mathbf{0 , 2 0 7}$ & 9,7 & 44,91 & 55,09 & 0,245 \\
\hline Tarn & 9,21 & 48,87 & 51,13 & 0,231 & 10,13 & 44,87 & 55,13 & 0,256 \\
\hline Tarn-et-Garonne & 10,28 & 48,03 & 51,97 & 0,259 & 10,82 & 44,03 & 55,97 & 0,275 \\
\hline Var & 13,39 & 49,42 & 50,58 & $\mathbf{0 , 3 3 7}$ & 13,74 & 51,69 & 48,31 & 0,346 \\
\hline Vaucluse & 10,09 & 45,93 & 54,07 & 0,255 & 13,22 & 45,74 & 54,26 & $\mathbf{0 , 3 3 4}$ \\
\hline Vendée & 10 & 66,93 & 33,07 & 0,272 & 10,69 & 60,38 & 39,62 & 0,276 \\
\hline Vienne & 11,86 & 51,89 & 48,11 & 0,299 & 12,28 & 47,01 & 52,99 & $\mathbf{0 , 3 1}$ \\
\hline Haute-Vienne & 10,76 & 40 & 60 & 0,278 & 11,54 & 37,81 & 62,19 & $\mathbf{0 , 3 0 2}$ \\
\hline Vosges & 12,06 & 54,27 & 45,73 & 0,305 & 12,2 & 50,18 & 49,82 & $\mathbf{0 , 3 0 7}$ \\
\hline Yonne & 11,6 & 52,74 & 47,26 & 0,292 & 12,96 & 49,9 & 50,1 & 0,326 \\
\hline Territoire de Belfort & 10,39 & 46,58 & 53,42 & 0,262 & 12,24 & 43,57 & 56,43 & $\mathbf{0 , 3 1 1}$ \\
\hline Essonne & 10,62 & 46,45 & 53,55 & 0,268 & 13,55 & 43,48 & 56,52 & $\mathbf{0 , 3 4 5}$ \\
\hline Hauts-de-Seine & 12,86 & 50,54 & 49,46 & 0,324 & 15,31 & 48,81 & 51,19 & 0,386 \\
\hline Seine-Saint-Denis & 13,53 & 38,41 & 61,59 & 0,352 & 17,02 & 37,02 & 62,98 & 0,447 \\
\hline Val-de-Marne & 11,63 & 44,93 & 55,07 & 0,294 & 15,91 & 43,27 & 56,73 & 0,406 \\
\hline Val-d'Oise & 11,73 & 46,03 & 53,97 & 0,296 & 14,03 & 43,16 & 56,84 & 0,358 \\
\hline
\end{tabular}

\begin{tabular}{|c|c|c|}
\hline \multicolumn{3}{|c|}{ Indices de lisibilité } \\
\hline & 1974 & 1981 \\
\hline Moyenne & 0,306 & 0,337 \\
\hline variabilité & 0,183 & 0,192 \\
\hline
\end{tabular}

